\title{
On stationary solutions of a stochastic differential equation*
}

Dedicated to Professor A. Kobori on his sixtieth birthday

By

Kiyosi ITô and Makiko Nisıo

(Received July, 1964)

\begin{tabular}{|c|c|c|}
\hline \multicolumn{3}{|c|}{ CONTENTS } \\
\hline 1 & Introduction & 1 \\
\hline 2 & Inequalities Concerning Stochastic Integrals & 5 \\
\hline 3 & Totally Bounded Sets of Stochastic Processes & 7 \\
\hline 4 & The Approximate Sum of a Stochastic Integral ... & 11 \\
\hline 5 & One-sided Solutions (Theorem 1) $\ldots \ldots \ldots \ldots \ldots \ldots \ldots$ & 13 \\
\hline 6 & 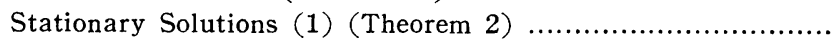 & 19 \\
\hline 7 & 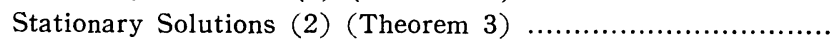 & 25 \\
\hline 8 & Stationary Solutions (3) (Theorem $4,5,6$ and 7 ) $\ldots \ldots$. & 28 \\
\hline 9 & $\begin{array}{l}\text { Borel Algebras Related to the Stationary Solutions } \\
(\text { Theorems } 8,9 \text { and } 10) \ldots \ldots \ldots \ldots \ldots \ldots \ldots \ldots \ldots \ldots \ldots \ldots \ldots \ldots \ldots \ldots \ldots\end{array}$ & 38 \\
\hline 10 & Lipschitz Condition (Theorems 11, 12 and 13) & 40 \\
\hline 11 & Linear Coefficients (1) (Theorems 14 and 15) & 47 \\
\hline 12 & Linear Coefficients (2) (Theorem 16) .. & 51 \\
\hline 13 & Diffusion (Theorems 17,18 and 19 ) & 56 \\
\hline 14 & A Modified Girsanov Example ......... & 70 \\
\hline 15 & A Deterministic Example ..... & 72 \\
\hline 16 & A Two-dimensional Example & 72 \\
\hline & Bibliography & 74 \\
\hline
\end{tabular}

\section{Introduction}

To begin with, let us introduce some preliminary notions.

Given a stochastic process $X(t),-\infty<t<\infty, \mathscr{B}_{u v}(X)$ denotes the least Borel algebra for which $X(t)$ is measurable for every $t \in[u, v]$.

* This work was supported in part by NSF 16319, NONR 225(28), AFOSR 49(638)1339 and the National Institutes of Health Grant $10452-02$ at Stanford University; by NONR 562(29) at Brown University. 
The Wiener process (Brownian motion) is denoted by $B(t)$, $-\infty<t<\infty$, and we normalize it as $B(0) \equiv 0$. In case we consider sevel Wiener processes at the same time, we shall write them as $B_{1}(t), B_{2}(t)$, etc. $\mathscr{B}_{u v}(d B)$ denotes the least Borel algebra for which $B(s)-B(t)$ is measurable for every $(t, s)$ with $u \leq t<s \leq v$.

The least Borel algebra that contains $\mathscr{B}_{1}, \mathscr{B}_{2}, \cdots$ is denoted by $\mathscr{B}_{1} \vee \mathscr{B}_{2} \vee \cdots$ or $\bigvee_{k} \mathscr{B}_{k}$

Given a stochastic process $X(t),-\infty<t<\infty$, and any fixed $s$, we shall call the stochastic process

$$
Y(\theta)=X(s+\theta), \quad \theta \leq 0,
$$

the past process of $X$ at time $s$ and denote it by $\pi_{s} X$.

$C_{\text {- }}$ denotes the space of all continuous functions defined on the negative half-line $(-\infty, 0] . C_{-}$is a metric space with the metric

$$
\rho_{-}(f, g)=\sum_{n=1}^{\infty} 2^{-n} \frac{\|f-g\|_{n}}{1+\|f-g\|_{n}}
$$

where $\|h\|_{n}=\max _{-n \leq t \leq 0}|h(t)|$.

Let $a(f)$ and $b(f)$ be continuous functionals defined on $C_{-}$. A stochastic process $X(t)$ is called a solution of a stochastic differential equation:

$$
d X(t)=a\left(\pi_{t} X\right) d t+b\left(\pi_{t} X\right) d B(t) \quad(-\infty<t<\infty),
$$

if

(1.4) $\mathscr{B}_{-\infty t}(X) \vee \mathscr{B}_{-\infty t}(d B)$ is independent of $\mathscr{M}_{t \infty}(d B)$ for every $t \in(-\infty, \infty)$,

and if

$$
\begin{array}{r}
X(t)-X(s)=\int_{s}^{t} a\left(\pi_{\theta} X\right) d \theta+\int_{s}^{t} b\left(\pi_{\theta} X\right) d B(\theta) \\
\text { for }-\infty<s<t<\infty .
\end{array}
$$

A solution $X(t)$ of (1.3) is called a stationary solution if $X(t)$ and $B(t)$ are strictly stationarily correlated, i.e., if the probability law of the system

(1.6) $(X, d B) \equiv(X(t),-\infty<t<\infty, B(v)-B(u),-\infty<u<v<\infty)$ 
is invariant under the time shift. A stationary solution is clearly a strictly stationary process. We are here mainly concerned with stationary solutions.

Consider a stochastic integral equation

$$
X(t)=X(0)+\int_{0}^{t} a\left(\pi_{s} X\right) d s+\int_{0}^{t} b\left(\pi_{s} X\right) d B(s)
$$

with the past condition:

$$
X(t)=X_{-}(t), \quad t \leq 0
$$

where $X_{-}(t), t \leq 0$ is a given process. Since a solution of this equation satisfies (1.3) on $t \geq 0$, it is called a one-sided solution.

Now we shall describe the outline of our paper.

In Sections 2, 3 and 4 we prove some facts on stochastic integrals and on the topology of stochastic processes which will be useful in the subsequent sections.

In Section 5 we shall prove the existence of a one-sided solution under a condition which requires that the coefficients $a(f)$ and $b(f)$ grows with $f$ in at most linear order. This condition prevents the solution from blowing $u p$ in finite time.

Our method is almost the same as Skorokhod's [18] and uses the Prohorov-Skorokhod theory of the totally bounded sets of stochastic processes [13] [17]. Though we are discussing the case in which the coefficients depend not only on the present value of $X(t)$ but also on its past behavior, this generalization does not create any serious difficulty.

In Sections 6 and 7 we shall find out some conditions for the existence of a stationary solution in terms of the one-sided solution discussed in Section 5. Roughly speaking, if the one-sided solution has a bounded moment, then there exists a stationary solution.

For the proof we shift the probability law $P$ of the joint process of the one-sided solution $Y$ and $d B$ by $-s$ to define $P_{s}$, then take the time average of $P_{s}$ to define $Q_{s}=s^{-1} \int_{0}^{s} P_{u} d u$ and finally take a convergent subsequence of $Q_{t}$ to get the probability law governing the joint process of the stationary solution $X$ and $d B$. We can prove the existence of such convergent subsequence 
observing that $\left\{P_{s}, s>0\right\}$ and $\left\{Q_{s}, s>0\right\}$ are both totally bounded in the Prohorov metric.

The shifting and averaging method is well-known as a tool to find an invariant measure, but our method used here seems to be new in that we applied it to the probability measure on the function space to get a stationary solution.

In Section 8 we shall give some direct conditions for the existence of a stationary solution in terms of the coefficients $a(f)$ and $b(f)$. Our conditions require that $a(f)$ is of the form $-a_{0}(f) f(0)+a_{1}(f)$ where $a_{0}(f)$ is dominant in a certain sense. However, these sufficient conditions are far from necessary.

It seems to be an interesting problem to find out a nice weak sufficient condition for the existence of a stationary solution, even though it is not necessary.

The condition (1.4) is the minimum requirement to make the stochastic integral in (1.5) meaningful but it does not always imply

$$
\mathscr{B}_{-\infty t}(X) \subset \mathscr{B}_{-\infty t}(d B) \quad-\infty<t<\infty ;
$$

even a weaker condition :

$$
\mathscr{B}_{-\infty t}(X) \subset \mathscr{B}_{-\infty s}(X) \vee B_{s t}(d B) \quad-\infty<s<t<\infty
$$

does not follow from (1.4). Therefore it occurs usually that in order to solve (1.3) for a given Wiener process $B(t)$, we must enlarge the given probability measure space on which $B(t)$ is defined.

The relationship between $\mathscr{B}_{-\infty t}(X)$ and $B_{-\infty t}(d B)$ is discussed in Section 9, and the non-linear backward representation will be also mentioned in this connection.

In Section 10 we shall treat the case that the coefficients satisfy the Lipschitz condition. Even in this case we should impose almost the same condition as in the general cases mentioned above to prove the existence of a stationary solution. However, the solution obtained satisfies $\left(1.4^{\prime \prime}\right)$, or sometimes even $\left(1.4^{\prime}\right)$.

Sections 11 and 12 are devoted to the case that $a(f)$ and $b(f)$ are linear in $f$.

In Section 13 we shall give a complete picture of the possible 
stationary solutions of a stochastic differential equation of the Markov type (i.e., the coefficients $a(f)$ and $b(f)$ depending only on the present value $f(0)$ ) under the Lipschitz condition.

In the last three sections we shall mention some pathological examples in connection with the relationship between $\mathscr{B}_{-\infty t}(X)$ and $\mathscr{B}_{-\infty t}(d B)$.

We put the time parameter $t$ always in the bracket as $X(t)$ and the suffix $s$ used in $X_{s}$ or $X_{s}(t)$ is to be considered as a parameter to distinguish stochastic processes from each other. Therefore $X_{s}$ means the process itself, while $X_{s}(t)$ means the value of the process at time $t$.

Acknowledgement. We thank Professor S. Karlin at Stanford University and Professor M. Rosenblatt at Brown University for their kind support and valuable suggestions during our work on this paper.

\section{Inequalities Concerning Stochastic Integrals}

Let $U$ be a random variable, $Y(t)$ and $Z(t)$ stochastic processes and $B(t)$ a Wiener process, where the time parameter $t$ moves on a bounded interval $[u, v]$.

Suppose that

$$
\mathscr{B}(U) \vee \mathscr{B}_{u t}(Y, Z, d B)
$$

is independent of $\mathscr{B}_{t v}(d B)$ for every $t \in[u, v]$ and

$$
\int_{u}^{n} E[|Y|(s)] d s+\int_{u}^{n} E\left[Z(s)^{2}\right] d s<\infty
$$

Now define $X(t)$ by

$$
X(t)=U+\int_{u}^{t} Y(s) d s+\int_{u}^{t} Z(s) d B(s), \quad u \leq t \leq v .
$$

$X(t)$ is well defined and we have $\mathscr{B}_{u t}(X) \subset \mathscr{B}(U) \vee \mathscr{B}_{u t}(Y, Z, d B)$.

Then we have

LEMMA 2.1.

$$
E\left(X(v)^{4}\right) \leq E\left(X(u)^{4}\right)+4 \int_{u}^{v} E\left[\left|X(s)^{3} Y(s)\right|\right]\left(d s+6 \int_{u}^{v} E\left(X(s)^{2} Z(s)^{2}\right) d s\right.
$$

(Notice that both sides may be infinite.) 
Proof: Consider a random time

$$
\sigma_{A}=\sup \left\{t \in[u, v]: \sup _{u \leq s \leq t}|X(s)| \leq A\right\}
$$

if there exists no such $t$, then put $\sigma_{A}=u$. Since almost all paths of $X(t)$ are continuous, we have

$$
\left(\sigma_{A}>t\right) \in \mathscr{B}_{u t}(X)
$$

and

$$
P\left(\sigma_{A}=v \text { for sufficiently big } A\right)=1 .
$$

We shall define $U_{A}, Y_{A}(t), Z_{A}(t)$ and $X_{A}(t)$ as follows

$$
\begin{array}{crr}
U_{A}=U(|U| \leq A), & =A(U>A), \quad=-A r & (U<-A) \\
Y_{A}(t)=Y(t), & Z_{A}(t)=Z(t) & \left(t<\sigma_{A}\right) \\
Y_{A}(t)=Z_{A}(t)=0 & & \left(t \geq \sigma_{A}\right) \\
X_{A}(t)=U_{A}+\int_{u}^{t} Y_{A}(s) d s+\int_{u}^{t} Z_{A}(s) d B(s) . &
\end{array}
$$

The stochastic integral $\int Z_{A}(s) d B(s)$ is well defined by virtue of (2.6) and $X_{A}(t)=X(t)$ or $\pm A$ according as $t<\sigma_{A}$ or $t \geq \sigma_{A}$. Therefore it holds with probability 1 that

$$
\left|X_{A}(t)\right| \leq A, \quad \lim _{A \uparrow \infty} X_{A}(t)=X(t) \quad \text { for every } t .
$$

Using a formula on stochastic differentials [4], we get

(2.9) $\quad X_{A}(v)^{4}$

$$
=U_{A}^{4}+\int_{u}^{v}\left[4 X_{A}(s)^{3} Y_{A}(s)+6 X_{A}(s)^{2} Z_{A}(s)^{2}\right] d s+\int_{u}^{v} 4 X_{A}(s)^{3} Z_{A}(s) d B(s)
$$

By virtue of $\left|X_{A}(t)\right| \leq A$ and the assumption (2.2), we can take the expectation on both sides of $(2.9)$ to get

(2.10) $E\left(X_{A}(v)^{4}\right)$

$$
\begin{aligned}
& =E\left(U_{A}^{4}\right)+E\left(\int_{u}^{\prime \prime} 4 X_{A}(s)^{3} Y_{A}(s) d s\right)+E\left(\int_{u}^{u} 6 X_{A}(s)^{2} Z_{A}(s)^{2} d s\right) \\
& =E\left(U_{A}^{4}\right)+E\left(\int_{u}^{\sigma} 4 X(s)^{3} Y(s) d s\right)+E\left(\int_{u}^{\sigma} 6 X(s)^{2} Z(s)^{2} d s\right) \\
& \leq E\left(U^{4}\right)+E\left(\int_{u}^{u} 4\left|X(s)^{3} Y(s)\right| d s\right)+E\left(\int_{u}^{\prime \prime} 6 X(s)^{2} Z(s)^{2} d s\right) \\
& =E\left(X(u)^{4}\right)+4 \int_{u}^{v} E\left(\left|X(s)^{3} Y(s)\right|\right) d s+6 \int_{u}^{\prime \prime} E\left(X(s)^{2} Z(s)^{2}\right) d s
\end{aligned}
$$


Recalling $X(t)=\lim _{A \rightarrow \infty} X_{A}(t)$, we have

$$
\begin{aligned}
E\left(X(v)^{4}\right) & \leq \lim _{A \rightarrow \infty} E\left(X_{A}(v)^{4}\right) \\
& \leq E\left(X(u)^{4}\right)+4 \int_{u}^{u} E\left(\left|X(s)^{3} Y(s)\right|\right) d s+6 \int_{u}^{v} E\left(X(s)^{2} Z(s)^{2}\right) d s
\end{aligned}
$$

LEMMA 2.2. If $\int_{u}^{v} E\left(\left|X(s)^{3} Y(s)\right|\right) d s<\infty$, then

(2.11) $\quad E\left(X(v)^{4}\right)$

$$
\leq E\left(X(u)^{4}\right)+4 \int_{u}^{v} E\left(X(s)^{3} Y(s)\right) d s+6 \int_{u}^{v} E\left(X(s)^{2} Z(s)^{2}\right) d s .
$$

Proof: In (2.10) we get

$$
\begin{aligned}
& E\left(X_{A}(v)^{4}\right) \\
& \quad \leq E\left(U^{4}\right)+4 E\left(\int_{u}^{\sigma_{4}} X(s)^{3} Y(s) d s\right)+6 \int_{u}^{v} E\left(X(s)^{2} Z(s)^{2}\right) d s
\end{aligned}
$$

But

$$
\begin{aligned}
& X(s)^{3} Y(s) \geq-\left|X(s)^{3} Y(s)\right| \\
& \int_{u}^{v} E\left(\left|X(s)^{3} Y(s)\right|\right) d s<\infty
\end{aligned}
$$

and $\sigma_{A} \uparrow v$ as $A \uparrow \infty$. Therefore

$$
\lim _{A \uparrow \infty} E\left(\int_{u}^{\sigma} X(s)^{3} Y(s) d s\right)=E\left(\int_{u}^{v} X(s)^{3} Y(s) d s\right)
$$

The rest of the proof is the same as in Lemma 2.1.

Using $4 X^{3} Y \leq 3 X^{4}+Y^{4}$ and $2 X^{2} Z^{2} \leq X^{4}+Z^{4}$ we can also derive from Lemma 2.1

LEMMA 2.3.

$$
E\left(X(v)^{4}\right) \leq E\left(X(u)^{4}\right)+6 \int_{u}^{v} E\left(X(s)^{4}+Y(s)^{4}+Z(s)^{4}\right) d s .
$$

Using the Schwartz inequality, $4 X Y Z^{2} \leq X^{4}+Y^{4}+2 Z^{4}$ and $2 X^{2} Y^{2} \leq X^{4}+Y^{4}$, we can derive from Lemma 2.1

LEMMA 2. 4.

$$
E\left(X(v)^{4}\right) \leq E\left(X(u)^{4}\right)+8 \int_{u}^{v} \sqrt{E\left(X(s)^{4}\right)} \sqrt{E\left(X(s)^{4}+Y(s)^{4}+Z(s)^{4}\right)} d s .
$$

\section{Totally Bounded Sets of Stochastic Processes}

Let $S$ be a separable complete metric space with the metric $\rho$ and $\mathscr{B}_{p}(S)$ the topological Borel algebra of subsets of $S$, i.e., the 
least Borel algeara containing all open subsets of $S$.

A mapping $X(\omega)$ from a probability measure space $\Omega(\mathscr{B}, P)$ into $S$ is called an $S$-valued random variable if it is measurable in the sense that $\{\omega: X(\omega) \in B\} \in \mathcal{B}$ for every $B \in \mathscr{B}_{\rho}(S)$. The probability law $\mu_{X}$ of $X$ is defined as the probability measure

$$
\mu_{X}(B)=P\{\omega: X(\omega) \in B\} .
$$

Given two probability measures $\mu_{1}, \mu_{2}$ on $S\left(\Re_{\rho}(S)\right)$, the Prohorov distance $L\left(\mu_{1}, \mu_{2}\right)$ is defined as follows. Let $\varepsilon_{12}$ be the infimum of $\varepsilon$ such that, for every closed subset $F$ of $S$

$$
\mu_{1}(F)<\mu_{2}\left(U_{\varepsilon}(F)\right)+\varepsilon
$$

where $U_{\varepsilon}(F)$ is the $\varepsilon$-neighborhood of $F$. Define $\varepsilon_{21}$ by switching $\mu_{1}$ and $\mu_{2}$ in $\varepsilon_{12}$ and set

$$
L\left(\mu_{1}, \mu_{2}\right)=\max \left(\varepsilon_{12}, \varepsilon_{21}\right) .
$$

The law-metric between two $S$-valued random variables $X_{1}, X_{2}$ (whether or not they are defined on the same probability measure space) is defined as the Prohorov distance between their probability laws and is denoted by $L\left(X_{1}, X_{2}\right)$. Notice that $L\left(X_{1}, X_{2}\right)=0$ means that $X_{1}$ and $X_{2}$ has the same probability law.

Let $\chi(S)$ be the system of all $S$-valued random variables. We can define $L$-convergence, $L$-Cauchy sequence, etc. on $\chi(S)$.

If $X_{n}, n=1,2, \cdots$ and $X$ are all defined on the same probability measure space and if $P\left(\rho\left(X_{n}, X\right) \rightarrow 0\right)=1$, then $X_{n}$ is clearly an $L$-Cauchy sequence. The converse is also true in the following sense :

THEOREM OF Skorokhod [17]. If $X_{n}, n=1,2, \cdots$ (whether or not they are definned on the same probability measure space) is an LCauchy sequence, then we can construct a sequence $Y_{n}, n=1,2, \cdots$ and $Y$ on the same probability measure space such that

$$
L\left(Y_{n}, X_{n}\right)=0 \quad \text { and } \quad P\left(\rho\left(Y_{n}, Y\right) \rightarrow 0\right)=1 .
$$

This can be also stated as follows.

If $\mu_{n}$ is a Cauchy sequence of probability measures on $S\left(\mathscr{B}_{\rho}(S)\right.$ ) in the Prohorov metric, then we can construct a sequence $Y_{n}$, 
On stationary solutions of a stochastic differential equation 9 $n=1,2, \cdots$ and $Y$ on the same probability measure space such that and

$$
\mu_{Y_{n}}=\mu_{n}
$$

$$
P\left(\rho\left(Y_{n}, Y\right) \rightarrow 0\right)=1 .
$$

A subsystem $\Re=\left\{X_{\alpha}, \alpha \in A\right\}$ of $\chi(S)$ is called totally $L$-bounded if every infinite sequence $X_{\omega_{n}}, n=1,2, \cdots$ taken from $\mathscr{n}$ has an $L$-Cauchy subsequence.

TheOREM OF PROHOROv [13]. In order for $\mathscr{D}=\left\{X_{\alpha}, \alpha \in A\right\}$ to be totally L-bounded in $\chi(S)$, it is necessary and sufficient that for every $\varepsilon>0$, there exists a compact subset $K_{\varepsilon}$ of $S$ (independent of a) with

$$
P\left(X_{\alpha} \in K_{\varepsilon}\right)>1-\varepsilon \quad \text { for every } \alpha \in A .
$$

Let $S_{i}\left(\rho_{i}\right), i=1,2, \cdots, n$, be complete separable metric spaces. Then the direct $S=S_{1} \times S_{2} \times \cdots \times S_{n}$ is also a complete separable metric space with the metric

$$
\rho(x, y)=\sum_{i=1}^{n} \rho_{i}\left(x_{i}, y_{i}\right), \quad x=\left(x_{1}, \cdots, x_{n}\right), \quad y=\left(y_{1}, \cdots, y_{n}\right) \in S
$$

It is clear that $X=\left(X_{1}, X_{2}, \cdots, X_{n}\right) \in \mathcal{\chi}(S)$ if and only if $X_{i} \in \chi\left(S_{i}\right)$, $i=1,2, \cdots, n$.

Let $\Re=\left\{X_{\infty}=\left(X_{\infty, 1}, X_{\infty, 2}, \cdots, X_{\infty, n}\right), \alpha \in A\right\}$ be a subsystem of $\chi(S)$. Using Prohorov's theorem and recalling the fact that the direct product of compact sets and the projection of a compact set are also compact, we can easily see

LEMMA 3.1. $\eta$ is totally bounded if and only if $\Re_{i}=\left\{X_{\infty, i}\right.$; $\alpha \in A\}\left(\subset \chi\left(S_{i}\right)\right)$ is totally $L$-bounded for every $i=1,2, \cdots, n$.

Hereafter we are concerned with the metric spaces $C_{-}=$ $C(-\infty, 0], C_{+}=C[0, \infty)$ and $C=(-\infty, \infty)$. The metric $\rho_{-}$on $C_{-}$ was defined in Section 1, namely

$$
\begin{gathered}
\rho_{-}(f, g)=\sum_{n=1}^{\infty} 2^{-n} \frac{\|f-g\|_{n}}{1+\|f-g\|_{n}} \\
\|h\|_{n}=\max _{-n \leq t \leq 0}|h(t)|
\end{gathered}
$$

Similarly for $\rho_{+}$and $\rho$ with 


$$
\|h\|_{n}=\max _{0 \leq t \leq^{n}}|h(t)| \quad \text { or } \max _{-n \leq t \leq^{n}}|h(t)| .
$$

Using Kolmogorov's idea, Prohorov [13] obtained a useful criterion for $\Re \subset \chi(C)$ to be totally $L$-bounded. We shall state it in a rather restricted form which will be useful in Sections 4,5 and 6 .

LEMMA 3.2. $\Re \subset \chi(C)$ is totally bounded if there exist $c>0$ and $c_{n}>0, n=1,2, \cdots$ such that, for every $X \equiv(X(t), t \in(-\infty, \infty)) \in \mathscr{I}$, we have

$$
E\left[X(0)^{4}\right] \leq c
$$

and

$$
E\left[\left[|X(t)-X(s)|^{4}\right]<c_{n}|t-s|^{3 / 2} \quad \text { for }|t|,|s| \leq n .\right.
$$

Similarly for $C_{-}$and $C_{+}$.

The idea of the proof is as follows. Using Chebyshev's inequality, Borel-Cantelli's lemma and the usual technique of diadic division, we can prove that, for every $\varepsilon>0$, there exist $\gamma(\varepsilon)$ and $\gamma_{n}(\varepsilon), n=1,2, \cdots$ (independent of $X \in \Re$ ) with

$$
P\left[|X(0)| \leq \gamma(\varepsilon), \sup _{-n \leq t<s \leq n} \frac{|X(t)-X(s)|}{|t-s|^{1 / 16}} \leq \gamma_{n}(\varepsilon)\right]>1-\varepsilon
$$

for every $X \in \Re$.

Setting

$$
K_{\varepsilon}=\left\{f \in C ;|f(0)| \leq \gamma(\varepsilon), \sup _{-n \leq t<s \leq^{n}} \frac{|f(t)-f(s)|}{|t-s|^{1 / 16}} \leq \gamma_{n}(\varepsilon)\right\}
$$

we can write (3.6) as $P\left(X \in K_{\varepsilon}\right)>1-\varepsilon$. On the other hand AscoliArzela's theorem shows that $K_{\varepsilon}$ is a compact subset of $C(\rho)$. To complete the proof, it is enough to apply Prohorov's theorem stated above.

Using the same technique we can prove the following lemma which will be useful in Section 7.

LEMma 3.3. $\Omega \subset \chi(C)$ is totally bounded, if there exist $c>0$, $c_{n}>0, n=1,2, \cdots$ and $A_{n} \in \mathcal{B}(C), n=1,2, \cdots$ such that, for every $X=(X(t), t \in(-\infty, \infty)) \in \Re$, we have

$$
E\left[X(0)^{2}\right] \leq c
$$




$$
E\left[|X(t)-X(s)|^{4}, X \in A_{n}\right] \leq c_{n}|t-s|^{2} \text { for }|t|,|s| \leq n,
$$

and

(3.9) $\sum_{n}\left(1-P\left(X \in A_{n}\right)\right)$ is convergent uniformly in $X \in \Re$.

\section{The Approximate Sum of a Stochastic Integral}

In this section $a(f)$ and $b(f)$ denote continuous functions of $f \in C_{-}\left(\rho_{-}\right), A$ is a parameter set and $[u, v]$ is a bounded interval. For each $\alpha \in A$ we have a stochastic process $X_{\infty}=X_{\alpha}(t),-\infty<t<\infty$, with continuous paths and a Wiener process $B_{a}$ such that

(4.1) $\mathscr{B}_{-\infty t}\left(X_{\omega}\right) \vee \mathcal{B}_{u t}\left(d \mathscr{S}_{\omega}\right)$ is independent of $\mathscr{B}_{t v}\left(d B_{\omega}\right), \quad u \leq t \leq v$.

Then $a\left(\pi_{t} X_{\alpha}\right)$ and $b\left(\pi_{t} X_{\alpha}\right)$ are continuous in $t$ with probability 1 and so

$$
I_{\infty}=\int_{u}^{n} a\left(\pi_{t} X_{\infty}\right) d t+\int_{u}^{v} b\left(\pi_{t} X_{\omega}\right) d B_{a}(t)
$$

is well defined.

Let $L_{\alpha}(\Delta)$ be the approximate sum of $I_{\omega}$ for $\Delta \equiv\left\{u=u_{0}<u_{1}\right.$ $\left.<\cdots u_{n}=v\right\}$ :

$$
I_{\alpha}(\Delta)=\sum_{i} a\left(\pi_{u_{i-1}} X_{\alpha}\right)\left(u_{i}-u_{i-1}\right)+\sum_{i} b\left(\pi_{u_{i-1}} X_{\alpha}\right)\left(B_{\alpha}\left(u_{i}\right)-B_{\alpha}\left(u_{i-1}\right)\right) .
$$

It is clear that $I_{\alpha}(\Delta) \rightarrow I_{\alpha}$ in probability for each $\alpha$ as $\|\Delta\| \equiv$ $\left(u_{i}-u_{i-1}\right) \rightarrow 0$, i.e., there exists $\delta=\delta(\varepsilon, \alpha)$ such that $\|\Delta\|<\delta(\varepsilon, \alpha)$ implies $P\left(\left|I_{\alpha}(\Delta)-I_{\alpha}\right|>\varepsilon\right)<\varepsilon$.

Lemma 4.1. If $\left\{X_{\alpha}, \alpha \in A\right\}$ is totally L-bounded, then we can take $\delta=\delta(\varepsilon)$ independent of $\alpha$ such that

$$
\|\Delta\|<\delta(\varepsilon) \text { implies } P\left(\left|I_{\alpha}(\Delta)-I_{\alpha}\right|>\varepsilon\right)<\varepsilon
$$

for every $\alpha \in A$.

To prove this we shall first prove

Lemma 4.2. If $a(f)$ is bontinuous in $f \in C$, then $a\left(\pi_{t} f\right)$ is continuous in two variables $(t, f)$.

PROOF: Assume that $t_{n} \rightarrow t_{0}, f_{n} \rightarrow f_{0}$. Set $l=\sup \left(\left|t_{0}\right|,\left|t_{1}\right|, \cdots\right)$. Given any $m$ fixed, we have 


$$
\begin{aligned}
& \left\|\pi_{t_{n}} f_{n}-\pi_{t_{0}} f_{0}\right\|_{m} \\
& \quad \leq\left\|\pi_{t_{n}} f_{n}-\pi_{n} f_{0}\right\|_{m}+\left\|\pi_{t_{n}} f_{0}-\pi_{t_{0}} f_{0}\right\|_{m} \\
& \quad \leq\left\|f_{n}-f_{0}\right\|_{m+l}+\sup _{-m \leq t \leq 0}\left|f_{0}\left(t+t_{n}\right)-f_{0}\left(t+t_{0}\right)\right| \rightarrow 0 \quad(n \rightarrow \infty) .
\end{aligned}
$$

Therefore $\rho_{-}\left(\pi_{t_{n}} f_{n}, \pi_{t_{0}} f_{0}\right) \rightarrow 0$ and so $a\left(\pi_{t_{n}} f_{n}\right) \rightarrow a\left(\pi_{t_{0}} f_{0}\right)$.

Now we shall prove Lemma 4.1. Since $\left\{X_{\alpha}\right\}$ is totally $L$ bounded, there exists a compact subset $K(\varepsilon)$ of $C$ such that

$$
P\left(X_{\alpha} \in K\right)>1-\frac{\varepsilon}{2} \quad \text { for every } \quad \alpha \in A
$$

Since $a\left(\pi_{t} f\right)$ and $b\left(\pi_{t} f\right)$ are continuous in $(t, f)$ by Lemma 4.2, they are uniformly continuous in $u \leq t \leq v$ and $f \in K$, namely they exists $\delta=\delta(\eta)>0$ for $\eta>0$ such that

$$
|t-s|<\delta, \quad u \leq t, s \leq v, \quad \rho(f, g)<\delta, \quad f, g \in K
$$

implies

$$
\left|a\left(\pi_{t} f\right)-a\left(\pi_{s} g\right)\right|<\eta, \quad\left|b\left(\pi_{t} f\right)-b\left(\pi_{s} g\right)\right|<\eta,
$$

in particular

$$
|t-s|<\delta, \quad u \leq t, s \leq v, \quad f \in K
$$

implies

$$
\left|a\left(\pi_{t} f\right)-a\left(\pi_{s} f\right)\right|<\eta, \quad\left|b\left(\pi_{t} f\right)-b\left(\pi_{s} f\right)\right|<\eta .
$$

Using a step function $\mathscr{P}_{\Delta}(t)$ :

$$
\mathcal{P}_{\Delta}(t)=u_{i-1} \quad \text { on } \quad u_{i-1} \leq t<u_{i},
$$

$I_{\alpha}(\Delta)$ can be written in the integral form

$$
I_{a}(\Delta)=\int_{u}^{\prime \prime} a\left(\pi_{\varphi_{\Delta}(t)} X_{\infty}\right) d t+\int_{u}^{\prime \prime} b\left(\pi_{\varphi_{\Delta}(t)} X_{\omega}\right) d B_{a}(t)
$$

and so

$I_{\alpha}-I_{\alpha}(\Delta)=\int_{u}^{v}\left(a\left(\pi_{t}\left(X_{\infty}\right)-a\left(\pi_{\varphi_{\Delta}(t)} X_{\infty}\right)\right) d t+\int_{u}^{\prime \prime}\left(b\left(\pi_{t} X_{\infty}\right)-b\left(\pi_{\omega_{\Delta}(t)} X_{\infty}\right)\right) d B_{a}(t)\right.$

Writing $[\mathscr{P}]_{\eta}$ for $\mathcal{P}$ truncated by $\eta$, i.e.,

$$
[\mathcal{P}]_{\eta}=\mathscr{P} \quad(|\mathcal{P}| \leq \eta), \quad=0 \quad(|\boldsymbol{P}|>\eta)
$$

and setting

$$
J_{a}(\Delta)=\int_{u}^{v}\left[a\left(\pi_{t} X_{\infty}\right)-a\left(\pi_{\varphi_{\Delta}(t)} X_{a}\right)\right]_{\eta} d t+\int_{u}^{\prime \prime}\left[b\left(\pi_{t} X_{\infty}\right)-b\left(\pi_{\varphi_{\Delta}(t)} X_{a}\right)\right]_{\eta} d B_{a}(t),
$$


On stationary solutions of a stochastic differential equation 13 we can see that $\|\Delta\|<\delta(\eta)$ implies

$$
P\left(J_{\omega}(\Delta) \neq I_{\infty}-I_{\infty}(\Delta)\right) \leq P\left(X_{\infty} \notin K\right)<\frac{\varepsilon}{2}
$$

because $\left|\varphi_{\Delta(t)}-t\right|<\|\Delta\|<\delta(\eta)$. On the other hand, $\|\Delta\|<\delta(\eta)$ implies

$$
\begin{array}{r}
E\left[\left|J_{\alpha}(\Delta)\right|^{2}\right] \leq 2\left[\int_{u}^{v} \eta d t\right]^{2}+2 \int_{u}^{v} \eta^{2} d t \leq \eta^{2} \cdot c \\
\left(c=2(v-u)^{2}+2(v-u)\right)
\end{array}
$$

and so

$$
P\left(\left|J_{\omega}(\Delta)\right|>\varepsilon\right) \leq \frac{\eta^{2} c}{\varepsilon^{2}}=\frac{\varepsilon}{2} \quad \text { if } \quad \eta=\sqrt{\varepsilon^{3} / 2 c},
$$

Thus $\|\Delta\|<\delta\left(\sqrt{\varepsilon^{3} / 2}\right)$ implies that for every $\alpha \in A$,

$$
P\left(\left|I_{a}-I_{\alpha}(\Delta)\right|>\varepsilon\right) \leq P\left(J_{\omega}(\Delta) \neq I_{\omega}-I_{\omega}(\Delta)\right)+P\left(\left|J_{\omega}(\Delta)\right|>\varepsilon\right)<\varepsilon .
$$

\section{One-sided Solutions}

In this section we shall solve

$$
d X(t)=a\left(\pi_{t} X\right) d t+b\left(\pi_{t} X\right) d B(t) \quad(t \geq 0)
$$

with the past condition:

$$
X(t)=X_{-}(t) \quad(t \leq 0),
$$

where $X_{-}(t), t \leq 0$ is a given stochastic process with continuous paths and $B(t),-\infty<t<\infty$, is a Wiener process with $B(0)=0$. To do this, it is enough to solve the stochastic integral equation

$$
\left.X(t)=X(0)+\int_{0}^{t} a\left(\pi_{s} X\right) d s+\int_{0}^{t} b\left(\pi_{s} X\right) d B(s) \quad t \geq 0\right)
$$

with (5.2) and

$$
\mathscr{B}_{-\infty t}(X) \vee \mathscr{S}_{0 t}(d B) \text { is independent of } \mathscr{B}_{t \infty}(d B) \text { for }
$$

$$
\text { every } t \geq 0
$$

Let $\Omega(\mathscr{B}, P)$ be the probability measure space on which $X_{-}(t)$, $t \leq 0$ and $B(t),-\infty<t<\infty$ are defined. We can assume that

$$
\mathscr{B}_{-\infty 0}\left(X_{-}\right) \vee \mathscr{B}(d B)=\mathscr{B},
$$

since this does not make any essential restriction. 
In order to solve our equation, it might be necessary to enlarge $\Omega(\mathscr{B}, P)$, as we have explained in Section 1 .

Let us impose the following assumptions.

(A. 1) $a(f)$ and $b(f)$ are both continuous on $C_{-}\left(\rho_{-}\right)$.

(A. 2) There exist a positive number $M_{1}$ and a bounded measure $d K_{1}$ on $(-\infty, 0]$ such that

$$
|a(f)|+|b(f)| \leq M_{1}+\int_{-\infty}^{0}|f(t)| d K_{1}(t) .
$$

(A. 3) $E\left(X_{-}(t)^{4}\right) \leq c \quad t \leq 0$

with a constant $c$.

(A. 4) $\mathscr{B}_{-\infty 0}\left(X_{-}\right)$is indepenpent of $\mathscr{B}_{0 \infty}(B)$.

The assumption (A.4) is automatically necessary by (5.2) and (5.3).

THEOREM 1. (Existence of a one-sided solution). Under (A.1), (A.2), (A.3) and (A.4), we can find a solution $X(t)$ of the stochastic integral equation (5.1') with (5.2), (5.3) and

$$
E\left[X(t)^{4}\right] \leq \gamma e^{\gamma t} \quad(t \geq 0)
$$

where $\gamma$ is a positive constant.

Remark: As we can see in the proof given below, (A.2) can be replaced by a weaker condition:

(A. $\left.2^{\prime}\right)$ There exist a positive $M$ and a bounded measure $d K$ on $(-\infty, 0]$ such that $|a(f)|^{4}+|b(f)|^{4} \leq M+\left.\int_{-\infty}^{0} f(t)\right|^{4} d K(t)$.

We shall divide the proof into two steps.

(i) Approximate polygonal solutions. Take $h>0$ and define an approximate solution $X_{h}(t)$ by Cauchy's polygonal method.

$$
\begin{array}{rr}
X_{h}(t)=X_{-}(t) & t \leq 0 \\
= & X_{h}(n h)+a\left(\pi_{n h} X_{h}(t-n h)+b\left(\pi_{n h} X_{h}\right)(B(t)-B(n h))\right. \\
& n h \leq t \leq(n+1) h ; \quad n=0,1,2, \cdots
\end{array}
$$

Take the step function $\mathscr{P}_{h}(t)$ :

$$
\mathcal{P}_{h}(t)=n h \quad n h \leq t<(n+1) h ; \quad n=0,1,2, \cdots .
$$


On stationary solutions of a stochastic differential equation 15

Then $X_{h}(t)$ satisfies

$$
X_{h}(t)=X_{h}(0)+\int_{0}^{t} a\left(\pi_{\varphi_{h}(s)} X_{h}\right) d s+\int_{0}^{t} b\left(\pi_{\varphi_{h}(s)} X_{h}\right) d B(s) \quad(t \geq 0)
$$

LEMMA 5. 1.

$$
c_{h}(t) \equiv \sup _{s \geq t} E\left[X_{h}(t)^{4}\right]<\gamma e^{\gamma t}, \quad t \geq 0
$$

with a constant $\gamma=\gamma(M, d K, c)$ which does not depend on $h$.

Proof: We shall first show that

$$
c_{h}(t)<\infty \quad-\infty<t<\infty .
$$

Since $c_{h}(t)$ is increasing, it is enough to show (5.9) for $t=n h$, $n=0,1,2, \cdots$, by induction. It is clearly by (A.3) that $c_{h}(0) \leq c<\infty$. If $c_{n}(n h)<\infty$, then $c_{n}((n+1) h)<\infty$, because we have, for $n h \leq t \leq$ $(n+1) h$,

$$
\begin{aligned}
E\left(X_{h}(t)^{4}\right) & \leq 3^{3}\left[E\left(X_{h}(n h)^{4}+E\left(a\left(\pi_{n h} X_{h}\right)^{4}\right) h^{4}+3 E\left(b\left(\pi_{n h} X_{h}\right)^{4}\right) h^{2}\right]\right. \\
& \leq 3^{3}\left[c_{h}(n h)+\left(M+\|K\| c_{n}(n h)\right)\left(h^{4}+3 h^{2}\right)\right]<\infty
\end{aligned}
$$

by virtue of (5.5) and (A. $\left.2^{\prime}\right)$, where $\|K\|=\int_{-\infty}^{0} d K(t)$.

Applying Lemma 2.3 to (5.7), we have, for $t \geq 0$,

$$
\begin{aligned}
E\left(X_{h}(t)^{4}\right) & \leq E\left(X_{h}(0)^{4}\right)+6 \int_{0}^{t} E\left[X_{h}(s)^{4}+a\left(\pi_{\varphi_{n}(s)} X_{h}\right)^{4}+b\left(\pi_{\varphi_{h}(s)} X_{h}\right)^{4}\right] d s \\
& \leq c+6 \int_{0}^{t}\left[c_{h}(s)(1+\|K\|)+M\right] d s,
\end{aligned}
$$

and so

$$
c_{h}(t) \leq c+6 \int_{0}^{t}\left[c_{h}(s)(1+\|K\|)+M\right] d s .
$$

Since $c_{h}(t)<\infty$ for all $t \geq 0$, we have

$$
c_{h}(t) \leq c_{h}(t)+M(1+\|K\|)^{-1} \leq\left(c+M(1+\|K\|)^{-1} e^{6(1+|| K \|) t}\right.
$$

by the method of iteration. Setting

$$
\gamma=\max \left(c+M(1+|| K||)^{-1}, 6(1+|| K||)\right),
$$

we get (5.8).

LEMMA 5.2.

$$
\begin{aligned}
& E\left(\left(X_{h}(t)-X_{h}(s)\right)^{4}\right) \leq \gamma_{n}|t-s|^{3 / 2} \\
& 0 \leq s<t \leq n, \quad n=1,2, \cdots,
\end{aligned}
$$


where $\gamma_{n}$ is a constant which does not depend on $h$ but only on $n$, $M,\|K\|$ and $c$.

PrOOF: It follows from (5.7)

$$
X_{h}(t)-X_{h}(s)=\int_{s}^{t} a\left(\pi_{\varphi_{h}(u)} X_{h}\right) d u+\int_{s}^{t} b\left(\pi_{\varphi_{h}(u)} X_{h}\right) d B(u) .
$$

Applying Lemma 2.4 to this, we have, for $0 \leq s<t \leq n$,

$$
\begin{aligned}
& E\left[\left(X_{h}(t)-X_{h}(s)\right)^{4}\right] \\
& \quad \leq 8 \int_{s}^{t} \sqrt{E\left[\left(X_{h}(u)-X_{h}(s)\right)^{4}\right]} \\
& \quad \leq 8 \int_{s}^{t} \sqrt{\left.E\left[\left(X_{h}(u)-X_{h}(s)\right)^{4}+a\left(\pi_{\varphi_{h}(u)} X_{h}\right)^{4}+b\left(\pi_{\varphi_{h}(u)}(u)-X_{h}\right)^{4}\right]^{4}\right]} d u \\
& \quad \leq 8 \sqrt{M+(\|K\|} \sqrt{M+(\|K\|+16) c_{h}(u)} d u
\end{aligned}
$$

by Lemma 5.1 .

Using Lemma 5.1 again, we have

$$
\begin{aligned}
E\left[\left(X_{h}(t)-X_{h}(s)\right)^{4}\right] \leq 2^{4} E\left[X_{h}(t)^{4}+X_{n}(s)^{4}\right] & \leq 2^{5} \gamma e^{\gamma n} \\
(0 & \leq s<t<n)
\end{aligned}
$$

Putting this in the above integral, we get

$$
E\left[\left(X_{h}(t)-X_{h}(s)\right)^{\natural}\right] \leq \gamma_{n}^{\prime} \cdot(t-s) \quad(0 \leq s<t \leq n)
$$

with a constant $\gamma_{n}^{\prime}=\gamma_{n}^{\prime}(M, d K, c)$ independent of $h$ and putting this in the integral again, we get

$$
E\left[\left(X_{h}(t)-X_{h}(s)\right)^{4}\right] \leq \gamma_{n}(t-s)^{3 / 2}
$$

with another constant $\gamma_{n}=\gamma_{n}(m, d K, c)$ independent of $h$.

Applying Lemma 3.2 to the class of stochastic processes $\left\{X_{+, h} \equiv\left(X_{h}(t), t>0\right) ; h>0\right\}\left(\subset \chi\left(C_{+}\right)\right)$in view of Lemma 5.1 and Lemma 5.2 , we can see that $\left\{X_{+, h} ; h>0\right\}$ is totally $L$-bounded. It is evident that $\left\{X_{-, h} \equiv X_{-}: h>0\right\}\left(\subset \chi\left(C_{-}\right)\right)$is totally $L$-bounded. Since $X_{h}=\left(X_{-, h}, X_{+, h}\right)$, we have

LEMMA 5.3. $\left\{X_{h} ; h>0\right\}$ is totally L-bounded.

(ii) Finding a solution.

It is evident that $\left\{B_{h} \equiv B ; h>0\right\}$ and $\left\{X_{-, h} \equiv X_{-} ; h>0\right\}$ are 
On stationary solutions of a stochastic differential equation 17

totally $L$-bounded. Since $\left\{X_{h}: h>0\right\}$ is totally $L$-bounded by Lemma 5.3, $\left\{\left(X_{h}, B, X_{-}\right): h>0\right\}$ is also totally $L$-bounded by Lemma 3.1, so that we can find an $L$-Cauchy sequence $\left(X_{h(n)}, B, X_{-}\right)$, $n=1,2, \cdots$ with $h(n) \downarrow 0$. By Skorokhod's theorem (see Section 3), we can construct $\left(Y_{n}, B_{n}, Y_{-, n}\right), n=1,2, \cdots, \infty$ on a certain probability measure space such that

$$
\begin{gathered}
L\left(\left(Y_{n}, B_{n}, Y_{-, n}\right),\left(X_{h(n)}, B, X_{-}\right)\right)=0 \quad n=1,2, \cdots \\
P\left(\left(Y_{n}, B_{n}, Y_{-, n}\right) \rightarrow\left(Y_{\infty}, B_{\infty}, Y_{-, \infty}\right)\right)=1
\end{gathered}
$$

where the convergence is to be understood in the sense of the metric in $C \times C \times C_{-}$.

Since $L\left(\left(B_{n}, Y_{-, n}\right),\left(B, X_{-}\right)\right)=0$ by (5.11) and $P\left(\left(B_{n}, Y_{-, n}\right) \rightarrow\right.$ $\left.\left(B_{\infty}, Y_{-, \infty}\right)\right)=1$ by $(5.12)$, we get

$$
L\left(\left(B_{\infty}, Y_{-, \infty}\right),\left(B, X_{-}\right)\right)=0 .
$$

If we can prove that

(5.14) $\mathscr{B}_{-\infty t}\left(Y_{\infty}\right) \vee \mathscr{B}_{0 t}\left(d B_{\infty}\right)$ is independent of $\mathscr{B}_{t \infty}\left(d B_{\infty}\right)$,

(5.15) with probability 1 ,

$$
Y_{\infty}(t)=Y_{-, \infty}(t), \quad t \leq 0,
$$

(5.16) with proaability 1 ,

$$
Y_{\infty}(t)=Y_{\infty}(0)+\int_{0}^{t} a\left(\pi_{s} Y_{\infty}\right) d s+\int_{0}^{t} b\left(\pi_{s} Y_{\infty}\right) d B_{\infty}(s), \quad t \leq 0,
$$

and

$$
E\left(Y_{\infty}(t)^{4}\right) \leq \gamma e^{\gamma t}, \quad t \geq 0,
$$

then we can conclude that $X(t) \equiv Y_{\infty}(t)$ is a solution of (5.1) which is to be constructed, by identifying $\left(B_{\infty}, Y_{-, \infty}\right)$ with $\left(B, X_{-}\right)$as is justified by (5.13).

$\mathscr{B}_{-\infty, t}\left(X_{h}\right) \vee \mathscr{B}_{0 t}(d B)$ is independent of $\mathscr{B}_{t \infty}(d B)$ by the definition of $X_{h}$ and $\mathscr{B}_{-\infty t}\left(Y_{n}\right) \vee \mathscr{B}_{a s}\left(d B_{n}\right)$ is therefore independent of $\mathscr{B}_{t \infty}\left(d B_{n}\right)$ by virtue of (5.11) and so (5.14) holds by (5.12).

Since $Y_{\infty}(t)$ and $Y_{-, \infty}(t)$ are continuous with probability 1 , it is enough for the proof of (4.15) to prove

$$
P\left(Y_{\infty}(t)=Y_{-, \infty}(t)\right)=1 \quad \text { for each } t \leq 0 .
$$


But this is immediate from (5.11), (5.12) and the definition of $X_{h}$. By the same reason as above, it is enough for the proof of (5.16) to prove for each $t$ that

(5.18) with probability 1 ,

Set

$$
Y_{\infty}(t)=Y_{\infty}(0)+\int_{0}^{t} a\left(\pi_{s} Y_{\infty}\right) d s+\int_{0}^{t} b\left(\pi_{s} Y_{\infty}\right) d B_{\infty}(s) .
$$

$$
I_{n}=\int_{0}^{t} a\left(\pi_{s} Y_{n}\right) d s+\int_{0}^{t} b\left(\pi_{s} Y_{n}\right) d B_{n}(s)
$$

$$
\begin{aligned}
I_{n}(h)= & \int_{0}^{t} a\left(\pi_{\varphi_{h}}(s) Y_{n}\right) d s+\int_{0}^{t} b\left(\pi_{\varphi_{h}(s)} Y_{n}\right) d B_{n}(s) \\
= & \sum_{k=c}^{m=1} a\left(\pi_{k h} Y_{n}\right) \cdot h+a\left(\pi_{m h} Y_{n}\right) \cdot(t-m h) \quad(m h \leq t<(m+1) h) \\
& +\sum_{k=0}^{m-1} b\left(\pi_{k h} Y_{n}\right)[B((k+1) h)-B(k h)]+b\left(\pi_{m h} Y_{n}\right)[B(t)-B(m h)] \\
& (n=1,2, \cdots, \infty)
\end{aligned}
$$

Since $P\left(\rho\left(Y_{n}, Y_{\infty}\right) \rightarrow 0\right)=1$ by $(5.12), \quad\left\{Y_{n}, n=1,2, \cdots, \infty\right\}$ is totally $L$-bounded and we can use Lemma 4.1 to get $\delta(\varepsilon)$ such that $|h|<\delta(\varepsilon)$ implies

$$
P\left(\left|I_{n}(h)-I_{n}\right|>\varepsilon\right)<\varepsilon, \quad n=1,2, \cdots, \infty .
$$

Since $Y_{n}(t)=Y_{n}(0)+I_{n}(h(n))$ by (5.7), (5.11) and (5.20), we have

$$
\begin{aligned}
& P\left(\left|Y_{\infty}(t)-Y_{\infty}(0)-I_{\infty}\right|>6 \varepsilon\right) \\
& =P\left(\mid Y_{\infty}(t)-Y_{n}(t)\right)-\left(Y_{\infty}(0)-Y_{n}(0)\right)-\left(I_{\infty}-I_{n}(h(n)) \mid>6 \varepsilon\right) \\
& \leq P\left(\left|Y_{\infty}(t)-Y_{n}(t)\right|>\varepsilon\right)+P\left(\left|Y_{\infty}(0)-Y_{n}(0)\right|>\varepsilon\right) \\
& \quad+P\left(\left|I_{\infty}-I_{n}(h(n))\right|>4 \varepsilon\right)
\end{aligned}
$$

and so by $(5.12)$

$$
P\left(\left|Y_{\infty}(t)-Y_{\infty}(0)-I_{\infty}\right|>6 \varepsilon\right) \leq \lim _{n \rightarrow \infty} P\left(\left|I_{\infty}-I_{n}(h(n))\right|>4 \varepsilon\right) .
$$

But it holds by (5.21) that

$$
\begin{aligned}
& P\left(\left|I_{\infty}-I_{n}(h(n))\right|>4 \varepsilon\right) \\
& \leq P\left(\left|I_{\infty}-I_{\infty}(h)\right|>\varepsilon\right)+P\left(\left|I_{\infty}(h)-I_{n}(h)\right|>\varepsilon\right) \\
& \quad+P\left(\left|I_{n}(h)-I_{n}\right|>\varepsilon\right)+P\left(\left|I_{n}-I_{n}(h(n))\right|>\varepsilon\right) \\
& \leq 3 \varepsilon+P\left(\left|I_{\infty}(h)-I_{n}(h)\right|>\varepsilon\right)
\end{aligned}
$$


On stationary solutions of a stochastic differential equation 19 for $h=\delta(\varepsilon) / 2<\delta(\varepsilon)$ and $n \geq n_{0}(\varepsilon)$, where $n_{0}(\varepsilon)$ is the maximum number $n$ for which $h(n)<\delta(\varepsilon)$.

By (5.20), (5.12) and the continuity of $a\left(\pi_{s} f\right)$ and $b\left(\pi_{s} f\right)$ in $f$ (see Lemma 4.2 ), $I_{n}(h) \rightarrow I_{\infty}(h)$ with probability 1 as $n \rightarrow \infty$ by Lemma 4.1. Therefore

$$
\lim _{n \rightarrow \infty} P\left(\left|I_{\infty}-I_{n}(h(n))\right|>4 \varepsilon\right) \leq 3 \varepsilon
$$

and so

$$
P\left(\left|Y_{\infty}(t)-Y_{\infty}(0)-I_{\infty}\right|>6 \varepsilon\right) \leq 3 \varepsilon .
$$

Since $\varepsilon$ is arbitrary, this inequality implies (5.18).

Using Lemma 5.1, we have (5.17) as follows

$$
E\left(Y_{\infty}(t)^{4}\right) \leq \lim _{n \rightarrow \infty}\left(Y_{n}(t)^{4}\right)=\lim _{n \rightarrow \infty} E\left(X_{h(n)}(s)^{4}\right) \leq \gamma e^{\gamma s} .
$$

\section{Stationary Solutions (1)}

Consider a stochastic differential equation:

$$
d X(t)=a\left(\pi_{t} X\right) d t+b\left(\pi_{t} X\right) d B(t)
$$

under the assumptions (A. 1) and (A.2) (or (A. 2')) imposed in Section 5. Since $X_{-}(t) \equiv 0$ satisfies (A.3) and (A.4) in that section, Theorem 1 shows that there exists a one-sided solution of (6.1) with the past condition :

$$
X(t) \equiv 0 \quad(t \leq 0)
$$

The solution constructed in this theorem satisfies

$$
E\left[X(t)^{4}\right] \leq \gamma e^{\gamma t} \quad(t>0)
$$

with a constant $\gamma \in(0, \infty)$.

No we shall prove

THEOREM 2. If the one-sided solution $X(t)$ mentioned above is bounded in the 4-th moment, i.e.,

$$
E\left[X(t)^{4}\right] \leq \alpha \quad(t \geq 0)
$$

with a donstant $\alpha \in(0, \infty)$, then there exists a stationary solution of (6.1). (See Section 1 for the definition of a stationary solution.) 
PROOF: Write $X_{s}(t)$ and $B_{s}(t)$ for $X(s+t)$ and $B(s+t)-B(s)$, respectively. Then we have

$$
\begin{gathered}
d X_{s}(t)=a\left(\pi_{t} X_{s}\right) d t+b\left(\pi_{t} X_{s}\right) d B_{s}(t) \quad t \geq-s \\
X_{s}(t) \equiv 0 \quad t \leq-s
\end{gathered}
$$

and $B_{s}$ is clearly a Wiener process with $B_{s}(0)=0$.

Let $P_{s}$ denote the probability law of $\left(X_{s}, B_{s}\right)$ which is a probability measure on $C^{2}(=C \times C)$ and $\theta_{s}$ the shift operator on $C^{2}$, i.e.,

$$
(\tilde{f}, \tilde{g})=\theta_{s}(f, g)
$$

if and only if $\tilde{f}(t)=f(s+t), \tilde{g}(t)=g(s+t)-g(s)$. Noticing that $\left(X_{s}, B_{s}\right)=\theta_{s}(X, B) \equiv \theta_{s}\left(X_{0}, B_{0}\right)$, we have

$$
P_{s}(E)=P_{0}\left(\theta_{-s} E\right) .
$$

Since $\pi_{s} f$ is continuous in $(s, f) \in(-\infty, \infty) \times C$, it is easy to see that $P_{s}(E)$ is Borel-measurable in $s$ for every $E \in \mathscr{B}\left(C^{2}\right)$. Therefore

$$
Q_{T}(E)=\frac{1}{T} \int_{0}^{T} P_{s}(E) d s
$$

is also a probability measure on $\left(C^{2}, \mathscr{S}\left(C^{2}\right)\right)$.

$B_{s}$ is clearly totally $L$-bounded by Lemma 3.2 , because $E\left(B_{s}(0)^{4}\right)=0$ and

$$
E\left(\left(B_{s}(v)-B_{s}(u)\right)^{4}\right)=3(v-u)^{2} \leq 3 \sqrt{2 n}|v-u|^{3 / 2}
$$

for $|u|,|v| \leq n$.

To prove that $\left\{X_{s}, s>0\right\}$ is also totally $L$-bounded, we shall verify

$$
\begin{gathered}
E\left(X_{s}(t)^{4}\right) \leq \alpha \\
E\left(\left(X_{s}(v)-X_{s}(u)\right)^{4}\right) \leq \gamma|v-u|^{3 / 2}
\end{gathered}
$$

with some constant $\gamma . \quad(6.7)$ is clear by (S). Since $X_{s}(t)=X(s+t)$, it is enough to prove that

$$
E\left((X(v)-X(u))^{4}\right) \leq \gamma|v-u|^{3 / 2} .
$$

Noticing $X(t) \equiv 0$ for $t \leq 0$, it is also enough to prove (6.9) for 
On stationary solutions of a stochastic differential equation 21 $v>u \geq 0$. Since $X$ satisfies $(6.1)$, we have

$$
X(v)-X(u)=\int_{u}^{v} a\left(\pi_{t} X\right) d t+\int_{u}^{v} b\left(\pi_{t} X\right) d B(t) .
$$

Using Lemma 2.4, we get

$$
\begin{aligned}
& E\left((X(v)-X(u))^{4}\right) \\
& \quad \leq 8 \int_{u}^{\prime \prime} \sqrt{E\left((X(t)-X(u))^{4}\right)} \sqrt{E\left((X(t)-X(u))^{4}+a\left(\pi_{t} X\right)^{4}+b\left(\pi_{t} X\right)^{4}\right)} d t \\
& \quad \leq 8 \sqrt{(16+\|K\|) \alpha+M} \int_{u}^{v} \sqrt{E\left((X(t)-X(u))^{4}\right)} d t .
\end{aligned}
$$

But

$$
E\left((X(t)-X(u))^{4}\right) \leq 16 \alpha
$$

by (S).

Replacing the integrand in the above integral with $\sqrt{16 \alpha}$, we get

$$
E\left((X(v)-X(u))^{4}\right) \leq \gamma_{1}(v-u) \quad\left(\gamma_{1}=\text { constant }\right)
$$

and replacing the integrand with $\sqrt{\gamma_{1}(t-u)}$, we have

$$
E\left((X(v)-X(u))^{4}\right) \leq \gamma(v-u)^{3 / 2} \quad(\gamma=\text { constant }) .
$$

Thus we have proved that both $\left\{X_{s}, s \geq 0\right\}$ and $\left\{B_{s}, s \geq 0\right\}$ are totally $L$-bounded. Hence it follows by Lemma 3.1 that $\left\{\left(X_{s}, B_{s}\right), s \geq 0\right\}$ is also totally $L$-bounded. Therefore we can find a compact subset $K=K(\varepsilon)$ of $C^{2}$ independent of $s$ for every $\varepsilon>0$ that $P_{s}(K)>1-\varepsilon$, so that $Q_{T}(K)>1-\varepsilon$. Since $K$ depends only on $\varepsilon,\left\{R_{T}, T>0\right\}$ is also totally bounded. Therefore there exists $T_{n} \uparrow \infty$ such that $Q_{T_{n}}$ converges to a certain probability measure $Q$ on $C^{2}$ in the Prohorov distance.

Let $(\tilde{X}, \tilde{B})$ be a $C^{2}$-valued random variable whose probability measure is $Q$. Now we shall prove that

(6.10) $\widetilde{B}(t)$ is a Wiener process with $\widetilde{B}(0)=0$,

(6.11) $\tilde{X}(t)$ is strictly stationarily correlated to $d \widetilde{B}$,

(6.12) $\mathscr{B}_{-\infty t}(\widetilde{X}, d \widetilde{B})$ is independent of $\mathscr{B}_{t \infty}(d \widetilde{B})$,

and

$$
d \tilde{X}(t)=a\left(\pi_{t} \tilde{X}\right) d t+b\left(\pi_{t} \tilde{X}\right) d \tilde{B}(t) \quad(-\infty<t<\infty)
$$


Once we prove this, we can see that $\tilde{X}(t)$ is a stationary solution which is to be sought, by identifying $\widetilde{B}$ and $B$.

Taking any bounded continuous function $\mathcal{P}$ on the $m$-space and observing

$$
\begin{aligned}
& E\left[\mathcal{P}\left(\widetilde{B}\left(t_{1}\right), \cdots, \widetilde{B}\left(t_{m}\right)\right)\right] \\
& \quad=\lim _{n \rightarrow \infty} \frac{1}{T_{n}} \int_{0}^{T_{n}} E\left[\mathcal{P}\left(B_{s}\left(t_{1}\right), \cdots, B_{s}\left(t_{m}\right)\right)\right] d s \\
& \quad=E\left[\mathcal{P}\left(B\left(t_{1}\right), \cdots, B\left(t_{m}\right)\right)\right],
\end{aligned}
$$

we can see that $\widetilde{B}(t)$ has the same probability law as $B(t)$, so that $\widetilde{B}(t)$ is a Wiener process with $\widetilde{B}(0)=0$.

Observing also, for any bounded continuous $\varphi$ and $\psi$ and for any fixed $t$,

$$
\begin{aligned}
& E\left[\varphi\left(\tilde{X}\left(t_{1}+t\right), \cdots, \tilde{X}\left(t_{m}+t\right)\right) \psi\left(\widetilde{B}\left(v_{1}+t\right)-\widetilde{B}\left(u_{1}+t\right), \cdots, \widetilde{B}\left(u_{k}+t\right)-\widetilde{B}\left(u_{k}+t\right)\right)\right] \\
& \quad=\lim _{n \rightarrow \infty} \frac{1}{T_{n}} \int_{0}^{T_{n}} E\left[\mathcal{P}\left(X_{s}\left(t_{1}+t\right), \cdots\right) \psi\left(B_{s}\left(v_{1}+t\right)-B_{s}\left(u_{1}+t\right), \cdots\right)\right] d s \\
& \quad=\lim _{n \rightarrow \infty} \frac{1}{T_{n}} \int_{0}^{T_{n}} E\left[\mathcal{P}\left(X_{s+t}\left(t_{1}\right), \cdots\right) \psi\left(B_{s+t}\left(v_{1}\right)-B_{s+t}\left(u_{1}\right), \cdots\right)\right] d s \\
& \quad=\lim _{n \rightarrow \infty} \frac{1}{T_{n}} \int_{t}^{T_{n}+t} E\left[\mathcal{P}\left(X_{s}\left(t_{1}\right), \cdots\right) \psi\left(B_{s}\left(v_{1}\right)-B_{s}\left(u_{1}\right), \cdots\right)\right] d s \\
& \quad=\lim _{n \rightarrow \infty} \frac{1}{T_{n}} \int_{0}^{T_{n}} E\left[\mathcal{P}\left(X_{s}\left(t_{1}\right), \cdots\right) \psi\left(B_{s}\left(v_{1}\right)-B_{s}\left(u_{1}\right), \cdots\right)\right] d s \\
& \quad=E\left[\mathcal{P}\left(\tilde{X}\left(t_{1}\right), \cdots\right) \psi\left(\widetilde{B}\left(v_{1}\right)-\widetilde{B}\left(u_{1}\right), \cdots\right)\right],
\end{aligned}
$$

we can see that $\tilde{X}$ and $d \tilde{B}$ are strictly stationarily correlated.

In order to prove (6.12) and (6.13), it is enough to show that, for every $S$,

$\left(6.12^{\prime}\right) \mathscr{B}_{-\infty t}(\tilde{X}) \vee \mathscr{B}_{-s t}(\tilde{X}, d \widetilde{B})$ is independent of $\mathscr{B}_{t c o}(d \widetilde{B})$,

$$
-S \leq t<\infty
$$

and

$$
\begin{array}{r}
\tilde{X}(t)=\tilde{X}(-S)+\int_{-S}^{t} a\left(\pi_{u} \tilde{X}\right) d u+\int_{-S}^{t} b\left(\pi_{u} \tilde{X}\right) d \tilde{B}(u), \\
-S \leq t<\infty
\end{array}
$$

Use $P_{s}$ and $T_{n}$ determined above to define the probability measures on $C^{2}$ : 
On stationary solutions of a stochastic differential equation 23

$$
Q_{S, T_{n}}(E)=\frac{1}{T_{n}-S} \int_{S}^{T_{n}} P_{s}(E) d s, \quad n \geq n_{0}(S)
$$

where $n_{0}(S)$ is the minimum number $n$ for which $T_{r}>S$.

For any bounded continuous function $f$ on $C^{2}$, we have

$$
\begin{aligned}
& \lim _{n \rightarrow \infty} \int_{C^{2}} f(\omega) Q_{S, T_{n}}(d \omega) \\
& \quad=\lim _{n \rightarrow \infty} \frac{1}{T_{n}-S} \int_{S}^{T_{n}} \int_{C^{2}} f(\omega) P_{s}(d \omega) d s \\
& \quad=\lim _{n \rightarrow \infty} \frac{1}{T_{n}} \int_{0}^{T_{n}} \int_{C^{2}} f(\omega) P_{s}(d \omega) d s \\
& \quad=\lim _{n \rightarrow \infty} \int_{C^{2}} f(\omega) Q_{T_{n}}(d \omega) \\
& \quad=\int_{C^{2}} f(\omega) Q(d \omega) .
\end{aligned}
$$

$Q_{S, T_{n}} \rightarrow Q$ in the weak* convergence and so in the Prohorov distance. Using Skorokhod's theorem, we can construct $\left(Y_{n}, \beta_{n}\right), n=n_{0}(S)$, $n_{0}(S)+1, \cdots, \infty$ on a certain probability measure space such that (6.15) the probability law of $\left(Y_{n}, \beta_{n}\right)$ in $Q_{S, T_{n}}$ for $n=n_{0}(S)$, $n_{0}(S)+1, \cdots$ and that of $\left(Y_{\infty}, \beta_{\infty}\right)$ is $Q$, and

$$
\left(Y_{n}, \beta_{n}\right) \rightarrow\left(Y_{\infty}, \beta_{\infty}\right) \text { in the metric in } C^{2}
$$

with probability 1 .

$\left(Y_{\infty}, \beta_{\infty}\right)$ and $(\tilde{X}, \widetilde{B})$ have the same probability law $Q$ and therefore $\beta_{\infty}$ is a Wiener process with $\beta_{\infty}(0)=0$.

$\beta_{n}$ in also a Wiener process with $\beta_{n}(0)=0$ for $n \geq n_{0}(S)$, because we have for any bounded continuous function $\rho\left(\xi_{1}, \cdots, \xi_{m}\right)$,

$$
\begin{aligned}
& E\left[\mathcal{P}\left(\beta_{n}\left(t_{1}\right), \cdots, \beta_{n}\left(t_{m}\right)\right)\right] \\
& \quad=\frac{1}{T_{n}-S} \int_{S}^{T_{n}} E\left[\mathcal{P}\left(B_{s}\left(t_{1}\right), \cdots, B_{s}\left(t_{m}\right)\right)\right] d s \\
& \quad=E\left[\mathcal{P}\left(B\left(t_{1}\right), \cdots, B\left(t_{m}\right)\right)\right]
\end{aligned}
$$

Now we shall prove

$\left(6.12^{\prime \prime}\right) \mathscr{B}_{-\infty t}\left(Y_{n}\right) \vee \mathscr{B}_{-S t}\left(Y_{n}, d \beta_{n}\right)$ is independent of $\mathscr{S}_{t \infty}\left(d \beta_{n}\right)$, and

$$
-S \leq t<\infty
$$


(6. $\left.13^{\prime \prime}\right) \quad Y_{n}(t)=Y_{n}(-S)+\int_{-S}^{t} a\left(\pi_{u} Y_{n}\right) d u+\int_{-S}^{t} b\left(\pi_{u} Y_{n}\right) d \beta_{n}(u)$,

with probability 1 .

$$
-S \leq t<\infty
$$

Noticing that $\mathscr{B}_{-\infty t}\left(X_{s}\right) \vee \mathscr{B}_{-s t}\left(X_{s}, d B_{s}\right)$ is independent of $\mathscr{B}_{t \infty}\left(d B_{s}\right)$ for $-S \leq t<\infty$ as far as $s \geq S$, and observing, for any bounded continuous $\varphi\left(\xi_{1}, \cdots, \xi_{p}, \eta_{1}, \cdots, \eta_{q}\right)$ and $\psi\left(\zeta_{1}, \cdots, \zeta_{r}\right)$ and for $s_{1}, \cdots, s_{p}$ $\leq t,-S \leq t_{1}, \cdots, t_{q} \leq t$ and $t \leq u_{1}, \cdots, u_{r}$,

$$
\begin{aligned}
E\left\{\mathcal{P}\left(Y_{n}\left(s_{1}\right), \cdots, Y_{n}\left(s_{p}\right), \beta_{n}\left(t_{1}, t\right), \cdots, \beta_{n}\left(t_{q}, t\right)\right) \psi\left(\beta_{n}\left(t, u_{1}\right), \cdots, \beta_{n}\left(t, u_{r}\right)\right)\right] \\
\quad=\frac{1}{T_{n}-S} \int_{S}^{T_{n}} E\left[\mathcal{P}\left(X_{s}\left(s_{1}\right), \cdots, B_{s}\left(t_{1}, t\right), \cdots\right) \psi\left(B_{s}\left(t, u_{1}\right), \cdots\right)\right] d s \\
\quad=\frac{1}{T_{n}-S} \int_{S}^{T_{n}} E\left[\mathcal{P}\left(X_{s}\left(s_{1}\right), \cdots, B_{s}\left(t_{1}, t\right), \cdots\right)\right] E\left[\psi\left(B_{s}\left(t, u_{1}\right), \cdots\right)\right] d s \\
\quad=\frac{1}{T_{n}-S} \int_{S}^{T_{n}} E\left[\mathcal{P}\left(X_{s}\left(s_{1}\right), \cdots, B_{s}\left(t_{1}, t\right), \cdots\right)\right] d s \cdot E\left[\psi\left(\beta_{n}\left(t, u_{1}\right), \cdots\right)\right] \\
\quad=E\left[\mathcal{P}\left(Y_{n}\left(s_{1}\right), \cdots, \beta_{n}\left(t_{1}, t\right), \cdots\right)\right] E\left(\psi\left(\beta_{n}\left(t, u_{1}\right), \cdots\right)\right],
\end{aligned}
$$

we can see that $\left(6.12^{\prime \prime}\right)$ is true where $\beta_{n}(u, v)$ and $B_{s}(u, u)$ stand for $\beta_{n}(v)-\beta_{n}(u)$ and $B_{s}(v)-B_{s}(u)$ respectively; in the computation above, we used the fact that both $B_{s}$ and $\beta_{n}$ are Wiener processes and so have the same probability law.

It is clear by the definition of $\left(X_{s}, B_{s}\right)$ that

$$
X_{s}(t)=X_{s}(-S)+\int_{-s}^{t} a\left(\pi_{u} X_{s}\right) d u+\int_{-s}^{t} b\left(\pi_{u} X_{s}\right) d B_{s}(u)
$$

with probability 1 , as far as $s<S$.

Noticing that both sides of (6.17) are $\mathscr{B}\left(X_{s}, B_{s}\right)$-measurable by the definition, there exist Borel measurable functions $\mathcal{P}$ and $\psi$ defined on $C^{2}$ such that, with probability $1, \mathcal{P}\left(X_{s}, B_{s}\right)$ and $\psi\left(X_{s}, B_{s}\right)$ are equal to the left and right sides respectively for each $t>-S$, as far as $s>S$. Therefore we have

$E\left(\left|\varphi\left(Y_{n}, \beta_{n}\right)-\psi\left(Y_{n}, \beta_{n}\right)\right|\right)=\frac{1}{T_{n}-S} \int_{S}^{T_{n}} E\left(\left|\mathcal{P}\left(X_{s}, B_{s}\right)-\psi\left(X_{s}, B_{s}\right)\right|\right) d s=0$

by (6. 17). Thus $\varphi\left(Y_{n}, \beta_{n}\right)=\psi\left(Y_{n}, \beta_{n}\right)$ with probability 1 and so (6. $\left.13^{\prime \prime}\right)$ holds with probability 1 for each $t \in(-S, \infty)$. But both sides of $\left(6.13^{\prime \prime}\right)$ are continuous in $t$ with probability 1 , we can see 
that the exceptional set of probability 0 can be taken indently of $t$. Thus (6.13") is proved.

Noticing (6.16), we can derive $\left(6.12^{\prime}\right)$ and $\left(6.13^{\prime}\right)$ from $\left(6.12^{\prime \prime}\right)$ and $\left(6.13^{\prime \prime}\right)$ respectively by the argument we used at the end of Section 5.

\section{Stationary Solutions (2)}

We shall find another condition for the existence of a stationary solution of the stochastic differential equation (6.1).

(A. 1) and (A.2) are assumed here as in the previous section. We can replace (A.2) by a weaker condition:

$$
\text { (A. } \left.2^{\prime \prime}\right) \quad|a(t)|^{2}+|b(f)|^{2} \leq M+\int_{-\infty}^{0}|f(t)|^{2} d K(t)
$$

with a positive constant $M$ and a bounded measure $d K$ on $(-\infty, 0]$. Since (A. $2^{\prime \prime}$ ) is stronger than (A. $2^{\prime}$ ) (with different $M$ and $d K$ ) in Section 5 , we can construct a one-sided solution $X(t)$ of (6.1) with the past condition : $X(t) \equiv 0, t \leq 0$ under the assumption (A.1) and (A.2) (or (A. $\left.2^{\prime \prime}\right)$ ), by virtue of Theorem 1 and the remark immediately following it.

Now we shall prove

THEOREM 3. If the one-sided solution $X(t)$ mentioned above is bounded in the second moment, i.e.,

$$
E\left(X(t)^{2}\right) \leq \alpha \quad(t \geq 0)
$$

with a constant $\alpha$, then there exists a stationary solution, provided dK has a compact support.

ProOF: Take $\sigma>0$ such that the support of $d K$ is contained in $[-\sigma, 0]$. The proof goes in the same way as in the proof of Theorem 2 except in proving the fact that $\left\{X_{s} \equiv \theta_{s} X ; s>0\right\}$ $\left(\theta_{s} X(t) \equiv X(s+t)\right)$ is totally $L$-bounded. To prove this, we shall use Lemma 3.3.

We shall start with two lemmas.

LEMMA 7.1. There exists $G=G(\varepsilon, l)$ such that

$$
P\left(\sup _{s \leq t \leq s+l}|X(t)|>G\right) \leq \varepsilon \quad \text { for every } s>0 \text {. }
$$


PROOF: Introducing two processes

$$
\grave{a}(t)=a\left(\pi_{t} X\right) \quad(t \geq 0), \quad=0 \quad(t<0)
$$

and

$$
\tilde{b}(t)=b\left(\pi_{t} X\right) \quad(t \geq 0), \quad=0 \quad(t<0),
$$

we obtain

$$
X(t)=X(s)+\int_{s}^{t} \tilde{a}(u) d u+\int_{s}^{t} \tilde{b}(u) d B(u) \quad(=\infty<t<\infty)
$$

Since

$$
\begin{aligned}
S \equiv & \sup _{s \leq t \leq S^{s+l}}|X(t)| \\
\leq & |X(s)|+\int_{s}^{s+l}|\tilde{a}(u)| d u+\sup _{s \leq t \leq \leq^{s+l}}\left|\int_{s}^{t} \tilde{b}(u) d B(u)\right| \\
& \quad(=U+V+W),
\end{aligned}
$$

we have

$$
P(S>G) \leq P\left(U>\frac{G}{3}\right)+P\left(V>\frac{G}{3}\right)+P\left(W>\frac{G}{3}\right) .
$$

Using (A. $\left.2^{\prime}\right),(\widetilde{S})$ and the property of stochastic integrals, we have

$$
\begin{aligned}
P\left(U>\frac{G}{3}\right) & \leq \frac{3 E(U)}{G} \leq \frac{3 \sqrt{E\left(X(s)^{2}\right)}}{G} \leq \frac{3 \sqrt{\alpha}}{G} \\
P\left(V>\frac{G}{3}\right) & \leq \frac{3 E(V)}{G} \leq \frac{3}{G} \int_{s}^{s+l} \sqrt{E(\hat{a}(u))^{2}} d u \\
& \leq \frac{3 l}{G} \sqrt{M+\|K\| \alpha}
\end{aligned}
$$

and

$$
P\left(W>\frac{G}{3}\right) \leq\left(\frac{3}{G}\right)^{2} \int_{s}^{s+l} E\left(\tilde{b}(u)^{2}\right) d u \leq \frac{9 l}{G^{2}}(M+\|K\| \alpha) .
$$

Therefore we get

$$
P(s>G) \leq \frac{\delta_{1}}{G}+\frac{\delta_{2} l}{G}+\frac{\delta_{3} l}{G^{2}} \quad\left(\delta_{i}: \text { constant }\right)
$$

and so we can find $G=G(\varepsilon, l)$ for which (7.1) holds.

LEMMA 7.2. There exists $\mu=\mu(G, l)$ such that for every $s$ and for every $u, v \in[s, s+l]$, we have

$$
E\left[(X(v)-X(u))^{4}, \sup _{s-\gamma \leq t \leq s+l}|X(t)| \leq G\right] \leq \mu|u-u|^{2} .
$$


Proof: We shall use $\tilde{a}(t)$ and $\tilde{b}(t)$ in the same meaning as above. We shall define $X_{G}(t)$ by truncating $X(t)$ by $G$, i.e.,

$$
X_{G}(t)=X(t) \text { or } 0
$$

according as $|X(t)| \leq G$ or not. Similarly we shall define $\tilde{a}_{G}(t)$ and $\tilde{b}_{G}(t)$ respectively by truncating $\tilde{a}(t)$ and $\tilde{b}(t)$ by $\alpha_{G}=\sqrt{M+\|K\| G}$. Now we shall consider the stochastic integral:

$$
L_{G}(u, v)=\int_{u}^{v} \tilde{a}_{G}(t) d t+\int_{u}^{v} \tilde{b}_{G}(t) d B(t)
$$

and observe

$$
\begin{gathered}
E\left[I_{G}(u, v)^{4}\right] \leq 8 E\left[\left(\int_{u}^{v} \tilde{a}_{G}(u) d t\right)^{4}\right]+8 E\left[\left(\int_{u}^{v} \tilde{a}_{G}(t) d B(t)\right)^{4}\right] \\
E\left[\left(\int_{u}^{v} \tilde{a}_{G}(u) d t\right)^{2}\right] \leq \alpha_{G}^{4}(v-u)^{4}
\end{gathered}
$$

and

$$
\begin{aligned}
E\left[\left(\int_{u}^{v} \tilde{b}_{G}(t) d B(t)\right)^{4}\right] & \leq 6 \int_{u}^{v} E\left[\left(\int_{u}^{t} \tilde{b}_{G}(s) d B(s)\right)^{2} \tilde{b}_{G}(t)^{2}\right] d t \\
& \quad \leq 6 \alpha_{G}^{2} \int_{u}^{v} E\left[\left(\int_{u}^{t} \tilde{b}_{G}(s) d B(s)\right)^{2}\right] d t \\
& \leq 6 \alpha_{G}^{2} \int_{u}^{v} \int_{u}^{t} E\left(\tilde{b}_{G}(s)^{2}\right) d s d t=3 \alpha_{G}^{4}(v-u)^{2} .
\end{aligned}
$$

Thus we get

$$
E\left(I_{G}(u, v)^{4}\right) \leq 8 \alpha_{G}^{4}\left(l^{2}+3\right)(v-u)^{2} \quad(s \leq u<v \leq s+l)
$$

If $\sup _{s-\sigma \leq t \leq s+l}|X(t)| \leq G$, then we have $X_{G}=X(t), \tilde{a}_{G}(t)=\tilde{a}(t), \tilde{b}_{G}(t)$ $=\tilde{b}(t)$ and so $I_{G}(u, v)=X(v)-X(u)$, as far as $t, u, v \in[s, s+l]$, because the support $d K$ is contained in $[-\sigma, 0]$. Thus we get

$$
\begin{gathered}
E\left[(X(v)-X(u))^{4}, \sup _{s-\sigma \leq t \leq s+l}|X(t)| \leq G\right] \\
\quad \leq E\left[I_{G}(u, v)^{4}\right] \leq 8 \alpha_{G}^{4}\left(l^{2}+3\right)(v-u)^{2},
\end{gathered}
$$

which proves Lemma 7.2.

Now let us prove that $\left\{X_{s}, s>0\right\}$ is totally $L$-bounded. Using Lemma 7.1, we have, for $G_{n}=G\left(2^{-n}, 2 n+\sigma\right)$,

$$
P\left(\sup _{-n-\sigma \leq t \leq n}\left|X_{s}(t)\right| \leq G_{u}\right)=P\left(\sup _{s-n-\sigma \leq t \leq s+n}|X(t)|<G_{u}\right)<1-2^{-n} .
$$


Using Lemma 7.2, we have, for $u, v \in[s-n, s+n]$

and so, for $u, v \in[-n, n]$

$$
\begin{array}{r}
E\left[(X(v)-X(u))^{4}: \sup _{s-n-\sigma \leq t \leq s+n}|X(t)| \leq G_{n}\right] \leq \gamma_{n}(a-u)^{2} \\
\left(\gamma_{n}=\mu\left(G_{n}, 2 n+\sigma\right)\right),
\end{array}
$$

$$
E\left[\left(X_{s}(v)-X_{s}(u)\right)^{4}, \sup _{-n-\sigma \leq t \leq n} \mid X_{s}(t) \leq G_{n}\right] \leq \gamma_{n}(v-u)^{2} .
$$

Writing $A_{n}$ for the set $\left.\left\{f \in C: \sup _{-n-\sigma \leq t \leq n} \mid f\right) \mid \leq G_{n}\right\}$, we have

$$
E\left[\left(X_{s}(v)-X_{s}(u)\right)^{4}, X_{s} \in A_{n}\right] \leq \gamma_{n}(v-u)^{2} \quad(u, v \in[-n, n])
$$

by $(7.4)$ and

$$
P\left(X_{s} \in A_{n}\right)>1-2^{-n} \quad(s>0)
$$

by (7.3). It is clear by $(\widetilde{S})$ that $E\left(X_{s}(t)^{2}\right) \leq \alpha$ for every $t \in(-\infty, \infty)$ and $s>0$. Thus we have proved that $\left\{X_{s}, s>0\right\}$ satisfies the assumptions in Lemma 3.3, so that it is totally $L$-bounded.

\section{Stationary Solutions (3).}

In the previous section 6 and 7 we presented some sufficient conditions for the existence of a stationary solution of the stochastic differential equation:

$$
d X(t)=a\left(\pi_{t} X\right) d t+b\left(\pi_{t} X\right) d B(t),
$$

using a one-sided solution of this equation with the past conditions: $X(t) \equiv 0, t \leq 0$; the existence of a one-sided solution was discussed in Section 5 .

In the present section we shall use the results obtained in Sections 6 and 7 to give sufficient conditions for the existence of a stationary solution in terms of the coefficients $a$ and $b$.

We shall begin with three lemmas.

LEMMA 8.1. If $r(t)$ and $\xi(t)$ are continuous on $[0, \infty)$, and if

$$
r(t)-r(s) \leq-\beta \int_{s}^{t} r(u) d u+\int_{s}^{t} \xi(u) d u \quad(0 \leq s<t<\infty),
$$

where $\beta$ is a positive constant, then

$$
r(t) \leq r(0)+\int_{0}^{t} e^{-\beta(t-u)} \xi(u) d u .
$$


On stationary solutions of a stochastic differential equation 29

PROOF : Write $r_{0}(t)$ for the integral appearing in (8.2) and set $r_{1}(t)=r(t)-r_{0}(t)-r(0) . \quad r_{0}(t)$ satisfies

$$
r_{0}^{\prime}(t)=-\beta r_{0}(t)+\xi(t)
$$

and so

$$
r_{0}(t)-r_{0}(s)=-\beta \int_{s}^{t} r_{0}(u) d u+\int_{s}^{t} \xi(u) d u .
$$

It follows from this and (8.1) that $r_{1}(t)$ is continuous and

$$
r_{1}(t)-r_{1}(s) \leq-\beta \int_{s}^{t} r_{1}(u) d u \text { and } r_{1}(0)=0 .
$$

To prove (8.2), it is enough to prove that $r_{1}(t) \leq 0$. If $r_{1}(t)>0$ for some $t$, then $r_{1}(0)=0$ implies that there exists an interval $\left[s_{1}, t_{1}\right] \subset[0, \infty)$ with $r_{1}\left(t_{1}\right)>r_{1}\left(s_{1}\right)$ and $r_{1}(t)>0$ on $\left[s_{1}, t_{1}\right]$, which contradict (8.3).

LEMMA 8.2. Suppose that $\rho(t)$ is continuous and satisfies

$$
\rho(t) \leq \alpha+\beta \int_{0}^{t} e^{-\gamma(t-s)} \rho(s) d s \quad(0 \leq t<\infty),
$$

weere $\alpha, \beta$ and $\gamma$ are all positive constants. If

$$
\gamma>\beta,
$$

then $\rho(t)$ is bounded; in fact,

$$
\rho(t) \leq \frac{\alpha \gamma}{\gamma-\beta}
$$

PROOF: It is easy to verify that

$$
\rho_{0}(t) \equiv \frac{\alpha}{\gamma-\beta}\left(\gamma-\beta e^{-(\gamma-\beta) t}\right)
$$

satisfies

$$
\rho_{0}(t)=\alpha+\beta \int_{0}^{t} e^{-\gamma(t-s)} \rho_{0}(s) d s \quad(0 \leq t<\infty) .
$$

Therefore $\rho_{1}(t) \equiv \rho(t)-\rho_{0}(t)$ satisfies

$$
\rho_{1}(t) \leq \beta \int_{0}^{t} e^{-\gamma(t-s)} \rho_{1}(s) d s \quad(0 \leq t<\infty) .
$$

Hence it follows by the method of iteration that 


$$
\rho_{1}(t) \leq \frac{\beta^{n} t^{n}}{n !} \max _{0 \leq s \leq t}\left|\rho_{1}(s)\right| \rightarrow 0 \quad(n \rightarrow \infty) .
$$

Thus we get $\rho(t) \leq \rho_{0}(t) \leq \alpha \gamma /(\gamma-\beta)$.

LEMMA 8.3. Suppose that $r(t)$ is continuous and non-negative on $[0, \infty)$ and satisfies

$$
r(t)-r(s) \leq \int_{s}^{t}(-\alpha \gamma(u)+\beta \sqrt{r(u)}+\gamma) d u \quad(0 \leq s<t<\infty),
$$

where $\alpha, \beta$ and $\gamma$ are constants and

$$
\alpha>0, \quad \gamma>0 \text {. }
$$

Then $r(t)$ is bounded.

PROOF : It is clear that $Q(\theta) \equiv-\alpha \theta^{2}+\beta \theta+\gamma<0$ on a certain half line $\left[\theta_{0}, \infty\right)$. We shall prove that $r(t) \leq \max \left(r(0), \theta_{0}^{2}\right)$. If $r(t)>$ $\max \left(r(0), \theta_{0}^{2}\right)$ for some $t \geq 0$, then there exists an interval $\left[r_{1}, t_{1}\right] \subset$ $[0, \infty]$ such that " $r\left(t_{1}\right)>r\left(s_{1}\right)$ " and " $\sqrt{r(t)}>\theta_{0}$, i.e., $Q(\sqrt{r(t)})>0$ on $\left[s_{1}, t_{1}\right]$ ". It follows from the second condition and (8.9) that

$$
r\left(t_{1}\right)-r\left(s_{1}\right) \leq \int_{s_{1}}^{t_{1}} Q(\sqrt{r(u)}) d u \leq 0
$$

which contradicts the first condition $r\left(t_{1}\right)>r\left(s_{1}\right)$.

Now we shall use Theorem 2 to derive

THEOREM 4. Suppose that $a(f)$ is of the form

$$
a(f)=-a_{0}(f) f(0)+a_{1}(f)
$$

with $a_{0}(f)$ and $a_{1}(f)$ continuous in $f \in C_{-}$and that $b(f)$ is continuous iu $f \in C_{-}$. Furthermore we assume the existence of positive constants $m, M, M_{1}$ and $M_{2}$ and bounded measures $d K_{1}$ and $d K_{2}$ on $(-\infty, 0]$ for which

$$
\begin{gathered}
m \leq a_{0}(f) \leq M \\
a_{1}(f)^{4} \leq M_{1}+\int_{-\infty}^{0} f(t)^{4} d K_{1}(t),
\end{gathered}
$$

and

$$
b(f)^{4} \leq M_{2}+\int_{-\infty}^{0} f(t)^{4} d K_{2}(t) .
$$


On stationary solutions of a stochastic differential equation 31 Then there exists a stationary solution of provided

$$
d X(t)=a\left(\pi_{t} X\right) d t+b\left(\pi_{t} X\right) d B(t),
$$

$$
m>\left\|\left.K_{1}\right|^{1 / 4}+\frac{3}{2}\right\| K_{2} \|^{1 / 2} .
$$

Remark. (8.13) can be replaced by

$$
\left|a_{1}(f)\right| \leq M_{1}+\int_{-\infty}^{0}|f(t)| d K_{1}(t)
$$

or

$$
\left|a_{1}(f)\right|^{2} \leq M_{1}+\int_{-\infty}^{0}|f(t)|^{2} d K_{1}(t),
$$

because each of these conditions implies (8.13) with different $M_{1}$ and $d K_{1}$. Similarly for (8.14).

ProOF: It follows from (8.11), (8.12) and (8.13) that

$$
\begin{aligned}
a(f)^{4} & \leq 8 a_{0}(f)^{4} f(0)^{4}+8 a_{1}(f)^{4} \\
& \leq 8 M^{4} f(0)^{4}+8 M_{1}+8 \int_{-\infty}^{0} f(t)^{4} d K_{1}(t) \\
& =\tilde{M}+\int_{-\infty}^{0} f(t)^{4} d \tilde{K}(t),
\end{aligned}
$$

where $\tilde{M}=8 M_{1}$ and $d \tilde{K}=8 d K_{1}+8 M^{4} \delta_{0} \quad\left(\delta_{0}=\right.$ the $\delta$-measure concentrated at 0$)$. By virtue of (8.16) and (8.14) we can use Theorem 1 (and the remark immediately following it) to find a one-sided solution with the past condition: $X(t) \equiv 0, t \leq 0$ such that $E\left(X(t)^{4}\right)$ $\leq \gamma e^{\gamma t}(-\infty<t<\infty)$ for some $\gamma>0$. To prove the existence of a stationary solution, it is enough to verify $(S)$ in Theorem 2 for this one-sided solution.

Write $r(t)$ for $E\left(X(t)^{4}\right)$ and set

$$
\rho(t)=\sup _{s \leq t} r(s) .
$$

It is clear that $r(t) \leq \rho(t) \leq \gamma e^{\gamma t}$. Now we shall prove that $\rho(t)$ is bounded.

By an assumption we have

$$
E\left[a\left(\pi_{t} X\right)^{4}\right] \leq \tilde{M}+\|\widetilde{K}\| \rho(t),
$$




$$
E\left[a_{1}\left(\pi_{t} x\right)^{4}\right] \leq M_{1}+\left\|K_{1}\right\| \rho(t)
$$

and

$$
E\left[b\left(\pi_{t} X\right)^{4}\right] \leq M_{2}+\left\|K_{2}\right\| \rho(t) .
$$

Applying Lemma 2.1 to

$$
X(t)-X(s)=\int_{s}^{t} a\left(\pi_{u} X\right) d u+\int_{s}^{t} b\left(\pi_{u} X\right) d B(u) \quad(0 \leq s \leq t),
$$

we get

$$
\begin{aligned}
& E\left[(X(t)-X(s))^{4}\right] \\
& \leq 4 \int_{s}^{t} E\left[\left|(X(u)-X(t))^{3} a\left(\pi_{u} X\right)\right|\right] d u+6 \int_{s}^{t} E\left[(X(u)-X(t))^{2} b\left(\pi_{u} X\right)^{2}\right] d u .
\end{aligned}
$$

Using

$$
\begin{aligned}
& 4\left|(X-Y)^{3} Z\right| \leq 3(X-Y)^{4}+Z^{4} \leq 24 X^{4}+24 Y^{4}+Z^{4}, \\
& 2(X-X)^{2} Z^{2} \leq(X-Y)^{4}+Z^{4} \leq 8 X^{4}+8 Y^{4}+Z^{4},
\end{aligned}
$$

(8.17) and (8.19), we get

$$
E\left[(X(t)-X(s))^{4}\right] \leq \int_{s}^{t}\left(\gamma_{1}+\gamma_{2} e^{\gamma u}\right) d u
$$

with some constants $\gamma_{1}$ and $\gamma_{2}$, and so

$$
\left|r(t)^{1 / 4}-r(s)^{1 / 4}\right|^{4} \leq E\left((X(t)-X(s))^{4}\right) \leq \int_{s}^{t}\left(\gamma_{1}+\gamma_{2} e^{\gamma u}\right) d u .
$$

This shows that $r(t)$ is continuous in $t \geq 0$.

By similar arguments we can easily see that $E\left(\left[\left|X(u)^{3} a\left(\pi_{u} X\right)\right|\right]\right.$, $E\left[\left|X(u)^{4} a_{0}\left(\pi_{u} X\right)\right|\right], E\left[\left|X(u)^{3} a_{1}\left(\pi_{u} X\right)\right|\right]$ and $E\left[X(u)^{2} b\left(\pi_{u} X\right)^{2}\right]$ are all bounded by a function of the form $\gamma_{1}+\gamma_{2} e^{\gamma t}$. Therefore we can apply Lemma 2.2 to

$$
X(t)=X(s)+\int_{s}^{t} a\left(\pi_{u} X\right) d u+\int_{s}^{t} b\left(\pi_{u} X\right) d B(u)
$$

to get

$$
\begin{aligned}
E\left(X(t)^{4}\right)-E\left(X(s)^{4}\right) & \\
\leq-4 \int_{s}^{t} E\left[a_{0}\left(\pi_{u} X\right) X(u)^{4}\right] d u & +4 \int_{s}^{t} E\left[X(u)^{3} a_{1}\left(\pi_{u} X\right)\right] d u \\
& +6 \int_{s}^{t} E\left[X(u)^{2} b\left(\pi_{u} X\right)^{2}\right] d u
\end{aligned}
$$




$$
\begin{aligned}
\leq-4 m \int_{s}^{t} E\left(X(u)^{4}\right) d u & +\int_{s}^{t} E\left[3 c \cdot X(u)^{4}+\frac{1}{c^{3}} a_{1}\left(\pi_{n} X\right)^{4}\right] d u \\
& +3 \int_{s}^{t} E\left[d \cdot X(u)^{4}+\frac{1}{d} b\left(\pi_{u} X\right)^{4}\right] d u,
\end{aligned}
$$

where $c$ and $d$ are positive constants and will be determined later; we used $4 X^{3} Y \leq 3 X^{4}+Y^{4}$ and $2 X^{2} Y^{2} \leq X^{4}+Y^{4}$ in the above observation.

Using the results obtained above, we have

$$
\begin{aligned}
& r(t)-r(s) \\
& \quad \leq-(4 m-3 c-3 d) \int_{s}^{t} r(u) d u+\int_{s}^{t}\left[k+\left(\frac{\left\|K_{1}\right\|}{c^{3}}+\frac{3\left\|K_{2}\right\|}{d}\right) \rho(u)\right] d u
\end{aligned}
$$

where $k=M_{1} / c^{3}+3 M_{2} / d$ (= constant).

If we assume that

$$
4 m-3 c-3 d>0 \text {, }
$$

then we can apply Lemma 8.1 to get

$$
r(t) \leq \int_{0}^{t} \exp [-(4 m-3 c-3 d)(t-u)] \cdot\left[k+\left(\frac{\left\|K_{1}\right\|}{c^{3}}+\frac{3\left\|K_{2}\right\|}{d}\right) \rho(u)\right] d u .
$$

Noticing that if $\xi(t)$ is increasing, then

$$
\int_{0}^{t} e^{-\beta(t-s)} \xi(s) d s \equiv \frac{1-e^{-\beta t}}{\beta} \xi(0)+\int_{0}^{t} \frac{1-e^{-\beta(t-s)}}{\beta} d \xi(s)
$$

is also increasing in $t$, we can see that the right side of (8.21) is also increasing, so that the left side of (8.21) can be replaced by $r(s)$ for every $s \leq t$ and so we get, with some constant $\tilde{k}$,

$$
\rho(t) \leq \tilde{k}+\left(\frac{\left\|K_{1}\right\|}{c^{3}}+\frac{3\left\|K_{2}\right\|}{d}\right) \int_{0}^{t} \exp [-(4 m-3 c-3 d)(t-u)] \rho(u) d u .
$$

Applying Lemma 8.2 to this, we can see that $\rho(t)$ is bounded, provided

$$
m>\frac{1}{4}\left(3 c+\frac{\left\|K_{1}\right\|}{c^{3}}\right)+\frac{3}{4}\left(d+\frac{\left\|K_{2}\right\|}{d}\right) ;
$$

notice that (8.23) includes (8.20). 
Setting $c=\left\|K_{1}\right\|^{1 / 4}$ and $d=\left\|K_{2}\right\|^{1 / 2}$, we can see that $\rho(t)$ is bounded under the condition (8.15); in fact (8.15) is the best condition among all conditions of the form (8.23).

As an application of Theorem 3 we shall prove

THEOREM 5. Suppose that $a(f)$ and $b(f)$ are written in the form:

$$
a(f)=a_{0}(f) f(0)+a_{1}(f), \quad b(f)=b_{0}(f) f(0)+b_{1}(f)
$$

with $a_{0}(f), a_{1}(f), b_{0}(f)$ and $b_{1}(f)$ continuous and bouhded in $f \in C_{-}$. Then it is sufficient for the existence of a stationary solution of the stochastic differential equation:

$$
d X(t)=a\left(\pi_{t} X\right) d t+b\left(\pi_{t} X\right) d B(t)
$$

that there exists a constant $m>0$ such that

$$
2 a_{0}(f)+b_{0}(f)^{2} \leq-m
$$

for every $f \in C_{-}$.

ProOF : By our assumption we have a constant $M$ which bounds $\left|a_{0}(f)\right|,\left|a_{1}(f)\right|,\left|b_{0}(f)\right|$ and $\left|b_{1}(f)\right|$ from above. Therefore

$$
|a(f)|+|b(f)| \leq 2 M+2 M|f(0)|,
$$

and so the conditions (A.1) and (A.2) in Theorem 3 are satisfied. To prove our theorem, it is enough to prove that a one-sided solution $X(t)$ with $X(t) \equiv 0, t \leq 0$, satisfies the condition that $E\left(X(t)^{2}\right)$ is bounded in $t \geq 0$; we can assume that $X(t)$ satisfies $E\left(X(t)^{4}\right) \leq \gamma e^{\gamma_{t}}$ for some constant $\gamma>0$.

Using a formula on stochastic integral [4], we have

$$
\begin{aligned}
X(t)^{2}-X(s)^{2}= & 2 \int_{s}^{t} X(u) a\left(\pi_{u} X\right) d u+2 \int_{s}^{t} X(u) b\left(\pi_{u} X\right) d B(u) \\
& +\int_{s}^{t} b\left(\pi_{u} X\right)^{2} d u
\end{aligned}
$$

Taking the expectations of both sides of (8.27) in view of

$$
\begin{aligned}
& E\left(\left|X(u) a\left(\pi_{u} X\right)\right|\right)+E\left(\left|X(u) b\left(\pi_{u} X\right)\right|\right) \\
& \quad \leq 2 M E\left(X(u)^{2}\right)+2 M E(|X(u)|) \leq M E\left(X(u)^{4}+1\right)+\frac{1}{2} M E\left(X(u)^{4}+3\right) \\
& \quad \leq \frac{5}{2} M+\frac{3}{2} M E\left(X(u)^{4}\right) \leq \frac{5}{2} M+\frac{3}{2} \gamma e^{\gamma u},
\end{aligned}
$$


we get, for $0 \leq s \leq t<\infty$,

(8.28) $E\left(X(t)^{2}\right)-E\left(X(s)^{2}\right)$

$$
\begin{aligned}
& =2 \int_{s}^{t} E\left(X(u) a\left(\pi_{u} X\right)\right) d u+\int_{s}^{t} b\left(\pi_{u} X\right)^{2} d u \\
& =\int_{s}^{t} E\left[\left(2 a_{0}\left(\pi_{u} X\right)+b_{0}\left(\pi_{u} X\right)^{2}\right) X(u)^{2}\right] d u \\
& \quad+2 \int_{s}^{t} E\left[\left(a_{1}\left(\pi_{u} X\right)+b_{1}\left(\pi_{u} X\right) b_{0}\left(\pi_{u} X\right)\right) X(u)\right] d u \\
& \quad+\int_{s}^{t} E\left[b_{1}\left(\pi_{u} X\right)^{2}\right] d u \\
& \leq \int_{s}^{t}\left[-m E\left(X(u)^{2}\right)+2\left(M+M^{2}\right) E(|X(u)|)+M^{2}\right] d u \\
& \leq \int_{s}^{t}\left[-m E\left(X(u)^{2}\right)+2\left(M+M^{2}\right) \sqrt{E\left(X(u)^{2}\right)}+M^{2}\right] d u .
\end{aligned}
$$

The continuity of $E\left(X(t)^{2}\right)$ follows from

$$
\begin{aligned}
& \left|E\left(X(t)^{2}\right)^{1 / 2}-E\left(X(s)^{2}\right)^{1 / 2}\right|^{2} \leq E\left[(X(t)-X(s))^{2}\right] \\
& \quad=2 \int_{s}^{t} E\left[(X(u)-X(s)) a\left(\pi_{u} X\right)\right] d u+\int_{s}^{t} E\left[b\left(\pi_{u} X\right)^{2}\right] d u
\end{aligned}
$$

which can be verified in the same way as above.

Therefore we can apply Lemma 8.3 to $r(t) \equiv E\left(X(t)^{2}\right)$ to see that $E\left(X(t)^{2}\right)$ is bounded.

As a second application of Theorem 3 we can prove the following theorem which is similar to Theorem 4 .

THEOREM 6. Under the same assumptions as in Theorem 4 except

$$
\begin{aligned}
& a_{1}(f)^{2} \leq M_{1}+\int_{-\infty}^{0} f(t)^{2} d K_{1}(t) \\
& b(f)^{2} \leq M_{2}+\int_{-\infty}^{0} f(t)^{2} d K_{2}(t)
\end{aligned}
$$

instead of (8.13) and (8.14), there exists a stationary solution of the same stochastic differential equation, provided

$$
m>\left\|K_{1}\right\|^{1 / 2}+\frac{1}{2}\left\|K_{2}\right\|
$$

and

(C) the supports of $d K_{1}$ and $d K_{2}$ are bounded. 
PROOF: Since the integrability of the integrals appearing here will be proved in the same way as in Theorem 4, we shall carry out only the formal computations.

Using Theorem 3 in view of $(C)$, we can see that it is enough to show the boundedness of $E\left(X(t)^{2}\right)$.

By virtue of a formula on stochastic integrals [4] we get $X(t)^{2}-X(s)^{2}=2 \int_{s}^{t} X(u) a\left(\pi_{u} X\right) d t+2 \int_{s}^{t} X(u) b\left(\pi_{u} X\right) d B(u)+\int_{s}^{t} b\left(\pi_{u} X\right)^{2} d u$ and so

$$
\begin{gathered}
E\left(X(t)^{2}-E\left(X(s)^{2}\right)=\int_{s}^{t} E\left[2 X(u) a\left(\pi_{u} X\right)+b\left(\pi_{u} X\right)^{2}\right] d u\right. \\
\leq-2 m \int_{s}^{t} E\left[X(u)^{2}\right] d u+\int_{s}^{t} E\left[2 X(u) a_{1}\left(\pi_{u} X\right)+b\left(\pi_{u} X\right)^{2}\right] d u \\
\leq-2 m \int_{s}^{t} E\left[X(u)^{2}\right] d u+\int_{s}^{t} E\left[c X(u)^{2}+\frac{1}{c} a_{1}\left(\pi_{u} X\right)^{2}+b\left(\pi_{u} X\right)^{2}\right] d u \\
\quad\left(c=\left\|K_{1}\right\|^{1 / 2}\right) .
\end{gathered}
$$

Setting $r(t)=E\left(X(t)^{2}\right)$ and $\rho(t)=\max _{0 \leq s \leq t} r(s)$, we get

$$
\begin{gathered}
r(t)-r(s) \\
\leq-2 m \int_{s}^{t} r(u) d u+\int_{s}^{t}\left[\left(c+\frac{1}{c}\left\|K_{1}\right\|+\left\|K_{2}\right\|\right) c(u)+\gamma\right] d u \\
\left(\gamma=\frac{1}{c} M_{1}+M_{2}\right) \\
=-2 m \int_{s}^{t} r(u) d u+\int_{s}^{t}\left[\left(2\left\|K_{1}\right\|^{1 / 2}+\left\|K_{2}\right\|\right) \rho(u)+\gamma\right] d u .
\end{gathered}
$$

Hence it follows by Lemma 8.1 that

$$
\begin{aligned}
r(t) & \leq \int_{0}^{t} e^{-2 m(t-s)}\left[\left(2\left\|K_{1}\right\|^{1 / 2}+\left\|K_{2}\right\|\right) \rho(s)+\gamma\right] d s \\
& \leq \frac{\gamma}{2 m}+\left(2\left\|K_{1}\right\|^{1 / 2}+\left\|K_{2}\right\|\right) \int_{0}^{t} e^{-2 m(t-s)} \rho(s) d s .
\end{aligned}
$$

Now use Lemma 8.2 to conclude that $r(t)$ is bounded if $m>\left\|K_{1}\right\|^{1 / 2}+\left\|K_{2}\right\| / 2$.

The following theorem is an immediate result from Theorem 4 and Theorem 6.

THEOREM 7. Under the same assumptions as in Theorem 4 except 
On stationary solutions of a stochastic differential equation 37

$$
\begin{aligned}
& \left|a_{1}(f)\right| \leq M_{1}+\int_{-\infty}^{0}|f(t)| d K_{1}(t) \\
& |b(f)| \leq M_{2}+\int_{-\infty}^{0}|f(t)| d K_{2}(t)
\end{aligned}
$$

instead of (8.13) and (8.14), there exists a stationary solution of the same stochastic differential equation, if

$$
m>\left\|K_{1}\right\|+\frac{3}{2}\left\|K_{2}\right\|^{2} .
$$

In case the supports of $d K_{1}$ and $d K_{2}$ are bounded, (8.15") can be replaced by a weaker condition:

$$
m>\left\|K_{1}\right\|+\frac{1}{2}\left\|K_{2}\right\|^{2} .
$$

Proof : By virtue $(X+Y)^{2}<\left(1+\frac{1}{\varepsilon}\right) X^{2}+(1+\varepsilon) Y^{2}(\varepsilon>0)$ and the Schwartz inequality, $\left(8.13^{\prime \prime}\right)$, and $\left(8.14^{\prime \prime}\right)$ imply

$$
\begin{aligned}
& \left|a_{1}(f)\right|^{2} \leq\left(1+\frac{1}{\varepsilon}\right) M_{1}^{2}+(1+\varepsilon)\left\|K_{1}\right\| \int_{-\infty}^{0}|f(t)|^{2} d K_{1}(t), \\
& |b(f)|^{2} \leq\left(1+\frac{1}{\varepsilon}\right) M_{2}^{2}+(1+\varepsilon)\left\|K_{2}\right\| \int_{-\infty}^{0}|f(t)|^{2} d K_{2}(t), \\
& \left|a_{1}(f)\right|^{4} \leq\left(1+\frac{1}{\varepsilon}\right)^{3} M_{1}^{4}+\left.(1+\varepsilon)^{3}|| K_{1}\right|^{3} \int_{-\infty}^{0}|f(t)|^{4} d K_{1}(t)
\end{aligned}
$$

and

$$
|b(f)|^{4} \leq\left(1+\frac{1}{\varepsilon}\right)^{3} M_{2}^{4}+\left.(1+\varepsilon)^{3}|| K_{2}\right|^{3} \int_{-\infty}^{0}|f(t)|^{4} d K_{2}(t) .
$$

If $\left(8.15^{\prime \prime}\right)$ holds, then we have

$$
m>(1+\varepsilon)^{3 / 4}|| K_{1}\left\|+\frac{3}{2}(1+\varepsilon)^{3 / 2}\right\| K_{2} \|^{2}
$$

i.e.,

$$
m>\left(\left.(1+\varepsilon)^{3}|| K_{1}\right|^{4}\right)^{1 / 4}+\frac{3}{2}\left[(1+\varepsilon)^{3} \|\left. K_{2}\right|^{4}\right]^{1 / 2}
$$

by taking small $\varepsilon>0$. Now we can apply Theorem 4 to prove first part of our theorem.

The second part will be proved similarly by Theorem 6 . 


\section{Borel Algebras Related to the Stationary Solution}

Let $X(t)$ be a stationary solution of the stochastic differential equation :

$$
d X(t)=a\left(\pi_{t} X\right) d t+b\left(\pi_{t} X\right) d B(t) .
$$

We shall prove some facts concerning the Borel algebras related to the solution $X$ and the Wiener process $B$.

The following three relations seem to be interesting:

$$
\begin{gathered}
\mathscr{B}_{-\infty t}(X) \subset \mathscr{B}_{-\infty t}(d B) \quad(-\infty<t<\infty), \\
\widehat{A}_{t} \mathscr{B}_{-\infty t}(X)=\mathscr{N}, \\
\mathscr{B}_{-\infty t}(X) \subset \mathscr{B}_{-\infty s}(X) \vee \mathscr{B}_{s t}(d B) \quad(-\infty<s<t<\infty),
\end{gathered}
$$

where $\Re$ is a triuial algebra which contains only sets of probability 0 or 1 .

It is evident that (9.2) implies (9.3).

In the general cases discussed in the previous sections we neither proved nor disproved these relations. In the next three sections we shall discuss some special cases in which (9.2) is true. In Section 13 we shall discuss the diffusion defined by a stochastic differential equation with coefficients satisfying the Lipschitz condition for which (9.4) holds but (9.3) does not always hold. In Section 14 we shall mention an example for which (9.4) does not hold. In Section 16 we shall give a two-dimensional stochastic differential equation for which both (9.3) and (9.4) are true but (9.2) is false.

If $X$ is a stationary solution of (9.1), then $X$ is strictly stationarily correlated to $d B$. In the following Theorems 8 and 9 we shall only assume this property.

The following theorem will be used in Section 16 and a similar fact for the discrete time parameter case was discussed by $M$. Rosenblatt [14] [15] [16].

THEOREM 8. Assume that $X$ is strictly stationarily correlated to $d B$.

(i) If (9.2) holds, then 
On stationary solutions of a stochastic differential equation 39

$$
E\left[\left|h(X(t))-E\left[h(X(t)) \mid \mathscr{S}_{0 t}(d B)\right]\right|\right] \rightarrow 0 \quad(t \rightarrow \infty)
$$

for every bounded Borel measurable $h(\xi)$ defined on $R^{1}$.

(ii) If (9.5) holds for one bounded monotone function $h$ (for example $h(\xi) \equiv \arctan \xi)$, then (9.2) holds.

Proof: It is enough to observe that Lévy-Doob's theorem shows

$$
E\left[h(X(0)) \mid \mathscr{B}_{-\infty 0}(d B)\right]=\lim _{t \rightarrow \infty} E\left[h\left(X(0) \mid \mathscr{B}_{-t 0}(d B)\right]\right.
$$

with probability 1 and that the strictly stationary correlation between $X$ and $d B$ implies

$$
\begin{aligned}
& E\left[\left|h(X(t))-E\left[h(X(t)) \mid \mathscr{B}_{0 t}(d B)\right]\right|\right] \\
& \left.\quad=E[\mid h(0))-E\left[h(X(0)) \mid \mathscr{B}_{-t_{0}}(d B)\right] \mid\right] .
\end{aligned}
$$

As an immediate result from the definitions we get

THEOREM 9. Assume that $X$ is strictly stationarily correlated to $d B$. If (9.2) holds, then there exists a Borel measurable functional $F$ on $C_{-}\left(\mathscr{B}\left(C_{-}\right)\right)$such that

$$
X(t)=F\left(\pi_{t} B\right)
$$

where

$$
\pi_{t} B(s)=B(s+t)-B(t), \quad s \leq 0 .
$$

Furthermore, if $E\left[X(0)^{2}\right]<\infty$, then $X(t)$ can be expanded in multiple Wiener integrals (see K. Itô [5] [6]):

(9. $\left.8^{\prime}\right) \quad X(t)=\sum_{n \geq 0} \int_{t_{1} \leq \cdots \leq i_{n} \leq n} \cdots \int_{n} f\left(t-t_{1}, t-t_{2}, \cdots, t-t_{n}\right) d B\left(t_{1}\right) \cdots d B\left(t_{n}\right)$.

We shall call (9.8) a backward representation of $X(t)$ and (9. $\left.8^{\prime}\right)$ a backward representation by multiple Wiener integrals (see N. Wiener [19]).

The representation (9.8) or (9.8') is called properly canonical (see M. Nisio [12]) if it holds that

$$
\mathscr{B}_{-\infty t}(X)=\mathscr{B}_{-\infty t}(d B) \quad(-\infty<t<\infty)
$$

By a theorem of Doob on stochastic integrals we can easily prove 
THEOREM 10. If $X(t)$ is a stationary solution of (9.1) satisfying (9.2) and if $b(f)>0$ (or at least $P\left(b\left(\pi_{0} X\right)>0\right)=1$ ), then $X(t)$ has a properly canonical backward representation. Furthermore if $E\left(X(0)^{2}\right)<\infty$, then $X(t)$ has a properly canonical backward representa tion by multiple Wiener integrals.

PROOF: It is enough to prove that

$$
B(t)-B(s)=\int_{s}^{t} b\left(\pi_{u} X\right)^{-1} d X(u)
$$

following Doob (see page 448 in his book [1]).

\section{Lipschitz Conditions}

In this section we shall discuss the stochastic differential equation :

$$
d X(t)=a\left(\pi_{t} X\right) d t+b\left(\pi_{t} X\right) d B(t)
$$

in case $a(f)$ and $b(f)$ satisfy the Lipschitz condition.

Let $C^{-}\left(d K_{1} d K_{2}\right)$ denote the subspace of $C_{-}$:

$$
\left\{f \in C_{-}: \int_{-\infty}^{0}\left|f(t) d K_{1}(t)+\int_{-\infty}^{0}\right| f(t) \mid d K_{2}(t)<\infty\right\}
$$

and $\mathscr{B}_{\rho_{-}}\left(C_{-}\left(d K_{1} d K_{2}\right)\right)$ the Borel algebra of sets of the form $C_{-}\left(d K_{1} d K_{2}\right) \wedge B, B \in \mathscr{B}_{\rho_{-}}\left(C_{-}\right)$. Notice that $C_{-}\left(d K_{1} d K_{2}\right)=C_{-}$in case the supports of $d K_{1}$ and $d K_{2}$ are bounded.

We shall first discuss one-sided solutions.

THEOREM 11. Suppose that $a(f)$ and $b(f)$ are measurable in $f \in C_{-}\left(d K_{1} d K_{2}\right)$ with respect to $\mathscr{B}_{\rho_{-}}\left(C_{-}\left(d K_{1} d K_{2}\right)\right)$ and satisfy the Lipschitz condition:

$$
|a(f)-a(g)|^{2} \leq \int_{-\infty}^{0}|f(t)-g(t)|^{2} d K_{1}(t)
$$

and

$$
|b(f)-b(g)|^{2} \leq \int_{-\infty}^{0}|f(t)-g(t)|^{2} d K_{2}(t)
$$

with bounded measures $d K_{1}$ and $d K_{2}$ on $(-\infty, 0]$. Then there exists one and only one solution of (10.1) with the past condition: 


$$
X(t)=X_{-}(t) \quad t \leq 0,
$$

where $X_{-}(t), t \leq 0$, is a given stochastic process independent of $\mathscr{B}_{00}(d B)$ and is bounded in the second order moment, i.e.,

$$
E\left[X_{-}(t)^{2}\right] \leq \alpha \quad(\alpha=\text { constant })
$$

Furthermore this solution satisfies

$$
\mathscr{B}_{-\infty t}(X) \subset \mathscr{B}_{-\infty 0}\left(X_{-}\right) \vee \mathscr{B}_{0 t}(d B)
$$

Remark 1. (L. 1) can be replaced by

$$
|a(f)-a(g)| \leq \int_{-\infty}^{0}|f(t)-g(t)| d \tilde{K}_{1}(t),
$$

because (L.1') implies (L.1) with $d K_{1}=\left\|\widetilde{K}_{1}\right\| d K_{1}$. Similarly for (L.2).

Remark 2. If $d K_{1}$ and $d K_{2}$ are concentrated at 0 , then $a\left(\pi_{s} X\right)$ and $b\left(\pi_{t} X\right)$ can be written as $\alpha(X(t))$ with $\beta(X(t))$ with $\alpha(\xi)$ and $\beta(\xi)$ satisfying the usual Lipschitz condition. This case was treated by K. Itô [8] in connection with the construction of diffusion processes attached to the infinitesimal generator:

$$
\mathcal{G}=a(\xi) \frac{d}{d \xi}+\frac{1}{2} b(\xi)^{2} \frac{d^{2}}{d \xi^{2}} .
$$

Since the method of the proof for this special case will work in our present case with no essential change, we shall give an outline of the proof of the existence only.

Proof of the existence part of Theorem 11. It is enough to solve the the stochastic integral equation

$$
X(t)=X(0)+\int_{0}^{t} a\left(\pi_{s} X\right) d s+\int_{0}^{t} b\left(\pi_{s} X\right) d B(s)
$$

with (10.2). To do this, we shall use the successive approximation.

Let us define a sequence of approximate solutions $X_{n}(t), n=0$, $1,2, \cdots$ as follows :

$$
\begin{aligned}
& \text { (10.6) } \quad X_{0}(t)=X_{-}(t) \\
& (t \leq 0) \\
& =X_{-}(0) \\
& (t \geq 0) \\
& \text { (10.7) } \quad X_{n}(t)=X_{-}(t) \\
& (t \leq 0) \\
& =X_{-}(0)+\int_{0}^{t} a\left(\pi_{s} X_{n-1}\right) d s+\int_{0}^{t} b\left(\pi_{s} X_{n-1}\right) d B(s) \quad(t \geq 0)
\end{aligned}
$$


The existence of the integral in (10.7) and the following inequality will be proved by induction:

$$
E\left[\left|X_{n}(t)-X_{n-1}(t)\right|^{2}\right] \leq \frac{\gamma_{1} \gamma_{2}^{n}(t+1)^{n} t^{n}}{n !} \quad(t>0, n=1,2, \cdots),
$$

where $\gamma_{1}$ and $\gamma_{2}$ are some constants determined by $\alpha,\left\|K_{1}\right\|,\left\|K_{2}\right\|$, $|a(0)|$ and $|b(0)|(0$ in $a(0)$ and $b(0)$ stands for the function identically equal to 0 on $(-\infty, 0])$.

Using the property of stochastic integrals, we get

$$
\begin{aligned}
& P\left(\sup _{0 \leq s \leq t}\left|X_{n+1}(t)-X_{r}(s)\right|>2 \varepsilon\right) \\
& \leq P\left(\int_{0}^{t}\left|a\left(\pi_{s} X_{n}\right)-a\left(\pi_{s} X_{n-1}\right)\right| d s>\varepsilon\right) \\
& \quad+P\left(\sup _{0 \leq^{s} \leq t}\left|\int_{0}^{t}\left(b\left(\pi_{s} X_{n}\right)-b\left(\pi_{s} X_{n-1}\right)\right) d B(s)\right|>\varepsilon\right) \\
& \leq \varepsilon^{-2} t \int_{0}^{t} E\left[\left(a\left(\pi_{s} X_{n}\right)-a\left(\pi_{s} X_{n-1}\right)\right)^{2}\right] d s \\
& \quad+\varepsilon^{-2} \int_{0}^{t} E\left[\left(b\left(\pi_{s} X_{n}\right)-\left(b\left(\pi_{s} X_{n-1}\right)\right)^{2}\right] d s\right. \\
& \leq \frac{\varepsilon^{-2} \gamma_{3} \gamma_{2}^{n}(t+1)^{n+1} t^{n+1}}{(n+1) !}
\end{aligned}
$$

with some constant $\gamma_{3}>0$.

Setting $\varepsilon=n^{-2}$ and $t=\log n$ in this inequality, we get

$$
\begin{aligned}
& P\left(\sup _{0 \leq s \log n}\left|X_{n+1}(s)-X_{n}(s)\right| \geq 2 n^{-2}\right) \\
& \quad \leq \frac{\gamma_{4} \gamma_{2}^{n+1}(\log n)^{n+1} n^{2}(1+\log n)^{n+1}}{(n-1) !} \quad\left(\gamma_{4}=\text { constant }>0\right)
\end{aligned}
$$

It is clear by Stirling's formula on $n$ ! that the right side of (10.9) is the $n$-th term of a convergent series. Hence it follows by Borel-Cantelli's lemma that

$$
P\left(\sum_{n} \sup _{r \leq s \leq \log n}\left|X_{n+1}(s)-X_{n}(s)\right|<\infty\right)=1,
$$

which shows that, with probability 1 ,

$$
X_{n}(s) \equiv X_{0}(s)+\sum_{k=1}^{n}\left(X_{k}(s)-X_{k-1}(s)\right), \quad n=1,2, \cdots
$$

is convergent uniformly on every bounded subinterval of $[0, \infty)$. 
Therefore the limit process $X(t) \equiv \lim _{n \rightarrow \infty} X_{n}(t)$ exists and is continuous in $t$ with probability 1.

(10.8) implies that

$$
\sum_{n}\left[E\left[\left(X_{n}(t)-X_{n-1}(t)\right)^{2}\right]\right]^{1 / 2}<\infty,
$$

so that

$$
E\left(\left(X(t)-X_{n}(t)\right)^{2} \rightarrow 0 \quad(n \rightarrow \infty) .\right.
$$

Letting $n$ tend to $\infty$ in (10.7) in view of (10.8), (10.9) and (10.10), we can conclude that $X(t)$ satisfies our stochastic integral equation with (10.2).

Since $\mathscr{B}_{-\infty t}\left(X_{n}\right) \subset \mathscr{B}_{-\infty t}\left(X_{-}\right) \vee \mathscr{B}_{t \infty}(d B)$ by the definition of $X_{n}$, (10.4) is obvious.

Now we shall discuss stationary solutions.

THEOREM 12. Suppose that $a(f)$ is of the form:

$$
a(f)=-c f(0)+a_{1}(f) \quad(c=\text { positive constant })
$$

and that $a_{1}(f)$ and $b(f)$ are measurable in $f \in C_{-}\left(d K_{1} d K_{2}\right)$ with respect to $\mathscr{B}_{\rho_{-}}\left(C_{-}\left(d K_{1} d K_{2}\right)\right)$. If following Lipschitz conditions are satisfied for $a_{1}$ and $b$ :

$$
\left|a_{1}(f)-a_{1}(g)\right|^{2} \leq \int_{-\infty}^{0}|f(t)-g(t)|^{2} d K_{1}(t)
$$

and

$$
|b(f)-b(g)|^{2} \leq \int_{-\infty}^{0}|f(t)-g(t)|^{2} d K_{2}(t)
$$

and if

$$
c>\left\|K_{1}\right\|^{1 / 2}+\frac{1}{4}\left\|K_{2}\right\|+\frac{1}{4}\left(\left\|K_{2}\right\|^{2}+8\left\|K_{1}\right\|^{1 / 2}\left\|K_{2}\right\|\right)^{1 / 2},
$$

then there exists one and only one stationary solution of the stochastic differential equation (10.1) with

$$
E\left(X(0)^{2}\right)<\infty .
$$

This solution satisfies $\mathscr{B}_{-\infty t}(X) \subset \mathscr{B}_{-\infty t}(d B)$ and has a backward representation by multiple Wiener integrals on $d B$ (see Section 9 for the definition of representations). 
ProOF :

(i) Existence. Consider the stochastic integral equation:

$$
X(t)=\int_{-\infty}^{t} e^{-c(t-s)} a_{1}\left(\pi_{s} X\right) d s+\int_{-\infty}^{t} e^{-c(t-s)} b\left(\pi_{s} X\right) d B(s) .
$$

Any solution of this equation satisfies (10.1), because it follows from (10.14) that

$$
\begin{gathered}
X(t)=e^{-c(t-s)} X(s)+e^{-c t} \int_{s}^{t} e^{c u} a_{1}(\pi X) d u+e^{-c t} \int_{s}^{t} e^{c u} b\left(\pi_{u} X\right) d B(u) \\
(s<t),
\end{gathered}
$$

so that a formula on stochastic differentials [4] shows that

$$
\begin{aligned}
d X(t)= & -c e^{-c(t-s)} X(s) d t-c e^{-c t} d t \int_{s}^{t} e^{c u} a_{1}\left(\pi_{u} X\right) d u+e^{-c t} e^{c t} a_{1}\left(\pi_{t} X\right) d t \\
& -c e^{-c t} d t \int_{s}^{t} e^{c u} b\left(\pi_{u} X\right) d B(u)+e^{-c t} e^{c t} b\left(\pi_{t} X\right) d B(t) \\
= & -c X(t) d t+a_{1}\left(\pi_{t} X\right) d t+b\left(\pi_{t} X\right) d B(t) \\
= & a\left(\pi_{t} X\right) d t+b\left(\pi_{t} X\right) d B(t) .
\end{aligned}
$$

Therefore if we can find a stationary process $X(t)$ which statisfies (10.14), then it will be a solution of (10.1).

In order to solve (10.14) we shall use the method of successive approximation. Define $X_{n}(t), n=0,1,2, \cdots$ as

$$
X_{0}(t) \equiv 0 \text {, }
$$

(10.10) $X_{n}(t)=\int_{-\infty}^{t} e^{-c(t-s)} a_{1}\left(\pi_{s} X_{n-1}\right) d s+\int_{-\infty}^{t} e^{-c(t-s)} b\left(\pi_{s} X_{n-1}\right) d B(s)$.

Let $\theta_{\tau}$ be the shift operator on the space of all random variables measurable with respect to $\mathcal{B}(d B)$ that is determined by

$$
\theta_{\tau} B(s, t)=B(s+\tau, t+\tau) \quad(B(s, t)=B(t)-B(s))
$$

The precise definition of $\theta_{\bar{\tau}}$ was given in Section 9 .

We shall easily verify the existence of the stochastic integral in (10.16) and the following properties of ${ }_{1} X_{n}(t)$ by induction:

$$
\begin{gathered}
\mathscr{B}_{-\infty t}\left(X_{n}\right) \subset \mathscr{B}_{-\infty t}(d B) \\
\theta_{\tau} X(t)=X_{n}(t+\tau)
\end{gathered}
$$

and 
On stationary solutions of a stochastic differential equation 45

$$
E\left[\left(X_{n}(t)-X_{n-1}(t)\right)^{2}\right]<\infty
$$

Setting $\rho_{n}=E\left[\left(X_{n}(t)-X_{n-1}(t)\right)^{2}\right]$, noticing that $\rho_{n}$ is finite by (10.21) and independent of $t$ by (10.21) and using $(X+Y)^{2} \leq(1+\varepsilon) X^{2}$ $+\left(1+\varepsilon^{-1}\right) Y^{2}$ and the Schwarz inequality, we get

$$
\begin{aligned}
\rho_{n+1}= & E\left[\left(X_{n+1}(t)-X_{n}(t)\right)^{2}\right] \\
\leq & E\left\{(1+\varepsilon)\left[\int_{-\infty}^{t} e^{-c(t-s)}\left(a\left(\pi_{s} X_{n}\right)-a\left(\pi_{s} X_{n-1}\right)\right) d s\right]^{2}\right\} \\
& +E\left\{\left(1+\varepsilon^{-1}\right)\left[\int_{-\infty}^{t} e^{-c(t-s)}\left(b\left(\pi_{s} X_{n}\right)-b\left(\pi_{s} X_{n-1}\right)\right) d B(s)\right]^{2}\right\} \\
\leq & {\left[(1+\varepsilon)\left(c^{2}\right)^{-1}\left\|K_{1}\right\|+\left(1+\varepsilon^{-1}\right)(2 c)^{-1}\left\|K_{2}\right\|\right] \rho_{n} \quad(\varepsilon>0) }
\end{aligned}
$$

Setting $\varepsilon=\left(c\left\|K_{2}\right\| / 2\left\|K_{1}\right\|\right)^{1 / 2}$ to get the best estimate :

$$
\rho_{n+1} \leq\left(\frac{1}{c}\left\|K_{1}\right\|^{1 / 2}+\frac{1}{\sqrt{2 c}}\left\|K_{2}\right\|\right)^{2} \rho_{n},
$$

we obtain

$$
\rho_{n+1} \leq A^{2 n} \rho_{1} \quad\left(A=\frac{1}{c}\left\|K_{1}\right\|^{1 / 2}+\frac{1}{\sqrt{2 c}}\left\|K_{2}\right\|^{1 / 2}\right)
$$

It is easy to see that " $A<1$ " is equivalent to $(10.12)$, so that $\rho_{n}$ tends to 0 exponentially fast under the assumption (10.22).

Noticing

$$
\begin{aligned}
& \sup _{|t| \leq T}\left|X_{n+1}(t)-X_{n}(t)\right| \\
& \leq e^{2 c T} \int_{-\infty}^{T} e^{-c(T-s)} \mid a_{1}\left(\pi_{s} X_{n}\right)-a_{1}\left(\pi_{s}\left(X_{n-1}\right) \mid d s\right. \\
& \quad+e^{2 c T} \sup _{-\infty \leq t \leq T}\left|\int_{-\infty}^{t} e^{-c(T-s)}\left(b\left(\pi_{s} X_{n}\right)-b\left(\pi_{s} X_{n-1}\right)\right) d B(s)\right|,
\end{aligned}
$$

we can easily see

$$
\begin{array}{r}
P\left(\sup _{|s| \leq \log n}\left|X_{n}(t)-X_{n-1}(t)\right|>n^{2 c} A^{n / 2}\right) \leq \gamma A^{-n} \rho_{n} \leq \gamma A^{n} \rho_{1} \\
(\gamma=\text { constant }) ;
\end{array}
$$

we should use here the fact that

$$
P\left[\sup _{s<t \leq t}\left|\int_{S}^{t} Y(s) d B(s)\right|>\varepsilon\right] \leq \varepsilon^{-2} \int_{S}^{T} E\left(Y(t)^{2}\right) d t
$$

is true even if $S=-\infty$, which can be easily seen as a limit of the usual case that $S>-\infty$. 
Since $\sum_{n} \gamma A^{n} \rho_{1}<\infty$ and $\sum_{n} n^{2 c} A^{n / 2}<\infty$, we can use PorelCantelli's lemma to see that with probability $1, X_{n}(t)$ converges uniformly on every bounded interval and that the limit process $X(t)$ is a solution of (10.14).

It follows at once from (10.18), (10.19) and (10.21) that $\mathscr{B}_{-\infty t}(X) \subset \mathcal{B}_{-\infty t}(d B), \theta_{\tau} X(t)=X(t+\tau)$ and $E\left(X(0)^{2}\right)<\infty$, so that $X(t)$ has a backward non-linear representation by multiple Wiener integrals.

Uniqueness. Let $X(t)$ be the stationary solution obtained above and $Y(t)$ any stationary solution. Using a formula on stochastic integrals [2], we can verify

$$
X(t)=e^{-c(t-s)} X(s)+\int_{s}^{t} e^{-c(t-u)} a_{1} \pi_{u}(X) d u+\int_{s}^{t} e^{-c(t-u)} b\left(\pi_{u} X\right) d B(u)
$$

and the same equation for $Y(t)$, from which we get

$$
\begin{aligned}
Y(t)-X(t)= & e^{-c(t-s)}(Y(s)-X(s))+\int_{s}^{t} e^{-c(t-s)}\left[a_{1}\left(\pi_{u} Y\right)-a_{1}\left(\pi_{u} X\right)\right] d u \\
& +\int_{s}^{t} e^{-c(t-s)}\left[b\left(\pi_{u} Y\right)-b\left(\pi_{u} X\right)\right] d B(u) ;
\end{aligned}
$$

notice that the integrals are meaningful, because $\mathscr{B}_{-\infty t}(X, Y, d B)$ $\left(\subset \mathscr{B}_{-\infty t}(Y, d B)\right)$ is independent of $\mathscr{B}_{t \infty}(d B)$,

$$
\begin{aligned}
E\left[\left|a_{1}\left(\pi_{u} Y\right)-a_{1}\left(\pi_{u} X\right)\right|^{2}\right] & \leq \int_{-\infty}^{0} E\left[|Y(u+v)-X(u+v)|^{2}\right] d K_{1}(u) \\
& \leq 2\left\|K_{1}\right\|\left[E\left(Y(0)^{2}\right)+E\left(X(0)^{2}\right)\right]
\end{aligned}
$$

and similarly

$$
E\left[\left|b\left(\pi_{u} Y\right)-b\left(\pi_{u} X\right)\right|^{2}\right] \leq 2\left\|K_{2}\right\|\left[E\left(Y(0)^{2}\right)+E\left(X(0)^{2}\right)\right] .
$$

Using (10.22) and the same method as in the proof of (10.21), we can see that $r(t)=E\left[(Y(t)-X(t))^{2}\right]$ and $\rho(t) \equiv \sup _{s \leq t} r(s)$ $\left(\leq 2\left[E\left(Y(0)^{2}\right)+E\left(X(0)^{2}\right)\right]<\infty\right)$ satisfy

$$
\begin{gathered}
r(t) \leq e^{-(t-t)} \rho(t)+(1+\varepsilon) \frac{1}{c^{2}}\left\|K_{1}\right\| \rho(t)+\left(1+\varepsilon^{-1}\right) \frac{1}{2 c}\left\|K_{2}\right\| \rho(t) \\
(t>s, \varepsilon>0) .
\end{gathered}
$$


On stationary solutions of a stochastic differential equation 47 Now letting $s$ tend to $-\infty$ in this inequality and taking the same $\varepsilon$ as before, we get

$$
r(t) \leq A^{2} \rho(t) \quad\left(A \equiv \frac{1}{c}\left\|K_{1}\right\|^{1 / 2}+\frac{1}{\sqrt{2 c}}\left\|K_{2}\right\|^{1 / 2}<1\right)
$$

and so

i.e.,

$$
r(s) \leq A^{2} \rho(s) \leq A^{2} \rho(t) \quad \text { for } \quad s \leq t
$$

$$
\rho(t) \leq A^{2} \rho(t) .
$$

Since $A<1$, we get $\rho(t)=0$, which shows $P(Y(t)=X(t))=1$ for each $t$. Hence it follows that

$$
P(Y(t)=X(t) \text { for every } t)=1,
$$

because $Y(t)$ and $X(t)$ are continuous, in $t$ with probability 1 . This completes the proof of Theorem 12 .

The following theorem which will be useful later is an immediate consequence of Theorem 12 by virtue of the Schwartz inequality.

THEOREM 13. In Theorem 9 we can replace (L. 1'), (L.2) and (10.14) with the following conditions

$$
\begin{aligned}
& \left|a_{1}(f)-a_{1}(g)\right| \leq \int_{-\infty}^{0}|f(t)-g(t)| d K_{1}(t) \\
& |b(f)-b(g)| \leq \int_{-+}^{0}|f(t)-g(t)| d K_{2}(t)
\end{aligned}
$$

and

$\left(10.14^{*}\right) \quad c>\left\|K_{1}\right\|+\frac{1}{4}\left\|K_{2}\right\|^{2}+\frac{1}{4}\left[\left\|K_{2}\right\|^{4}+8\left\|K_{1}\right\|\left\|K_{2}\right\|^{2}\right]^{1 / 2}$.

\section{Linear Coefficients (1).}

In this section we shall solve the stochastic differential equation :

$$
d X(t)=a\left(\pi_{t} X\right) d t+b\left(\pi_{t} X\right) d B(t)
$$

in case $a(f)$ and $b(f)$ are linear, i.e.,

$$
\begin{aligned}
& a(f)=M_{1}+\int_{-\infty}^{0} f(t) d K_{1}(t), \\
& b(f)=M_{2}+\int_{-\infty}^{0} f(t) d K_{2}(t),
\end{aligned}
$$


where $d K_{1}$ and $d K_{2}$ are bounded signed measures on $(-\infty, 0]$. $a(f)$ is defined for every Borel measurable function $f$ integrable on $(-\infty, 0]$ with respect to the total variation measure $\left|d K_{1}\right|$ of $d K_{1}$. Similarly for $b(f)$.

It is obvious that

$$
\begin{aligned}
& |a(f)| \leq\left|M_{1}\right|+\int_{-\infty}^{0}|f(t)|\left|d K_{1}(t)\right|, \\
& |b(f)| \leq\left|M_{1}\right|+\int_{-\infty}^{0}|f(t)|\left|d K_{2}(t)\right|,
\end{aligned}
$$

and

$$
\begin{aligned}
& |a(f)-a(g)| \leq \int_{-\infty}^{0}|f(t)-g(t)|\left|d K_{1}(t)\right|, \\
& |b(f)-b(g)| \leq \int_{-\infty}^{0}|f(t)-g(t)|\left|d K_{2}(t)\right| .
\end{aligned}
$$

Since the Lipschitz conditions for $a(f)$ and $b(f)$ are satisfied by (11.4), all the results obtained in the previous section hold here.

If the supports of $d K_{1}$ and $d K_{2}$ are bounded, then $a(f)$ and $b(f)$ are defined for every $f \in C_{\text {- }}$ and the condition (A.2) (or (A. $\left.2^{\prime}\right)$ ) is satisfied, so that the results in Section 5, 6, 7 and 8 hold here.

The following theorem for the stationary solutions holds only in our case of linear coefficients.

THEOREM 14. If $x(t)$ is any stationary solution of the stochastic differential equation (11.1) with $a(f)$ and $b(f)$ linear in $f$ and if $\gamma_{0}=E\left(X(0)^{2}\right)<\infty$, then $Y(t)=E[X(t) \mid \mathscr{B}(d B)]$ has a version with continuous paths which is a stationary solution of (11.1) and has a backward representation by multiple Wiener integrals.

PROOF: We shall use the following facts on the conditional expectation :

(C. 1) if $E\left(X^{2}\right)<\infty$, then $E\left[E(X \mid \mathcal{C})^{2}\right] \leq E\left(X^{2}\right)$,

(C.2) if $C \supset \mathcal{D}$, if $\theta$ is a measure preserving set transformation (up to measure 0 ) of $\mathcal{C}$ onto itself ( $\theta$ denoting also the transformation of the space of $C$-measurable function onto itself induced by the set transformation $\theta$ ) and if $X$ is $\mathcal{C}$-measurable and integrable, then

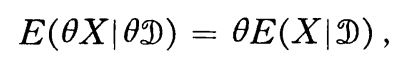




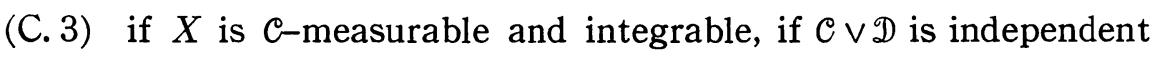
of $\mathscr{E}$, then $E(X \mid \mathcal{D} \vee \mathbb{E})=E(X \mid \mathcal{D})$.

(C.1) and (C.2) follow at once from the definitions. To prove (C.3), let $Y$ be bounded and $D$-measurable and $Z$ bounded and $\varepsilon$-measurable. Then

$$
\begin{aligned}
E[E(X \mid \mathcal{D} \vee \mathscr{E}) Y Z] & =E(X Y Z)=E(X Y) E(Z)=E[E(X \mid \mathcal{D}) Y] E(Z) \\
& =E[E(X \mid \mathfrak{D}) Y Z]
\end{aligned}
$$

which proves (C. 3 ).

Since $E\left[X(t)^{2}\right]=E\left[X(0)^{2}\right]=\gamma_{0}<\infty$, we have

$$
E\left[a\left(\pi_{t} X\right)^{2}\right] \leq \gamma_{1}, \quad E\left[b\left(\pi_{t} X\right)^{2}\right] \leq \gamma_{1}
$$

by $(11.3) ; \gamma_{1}, \gamma_{2}, \cdots$ stand for positive constants in this proof.

Using (11.5) and

$$
\begin{array}{r}
X(t)-X(s)=\int_{s}^{t} a\left(\pi_{u} X\right) d u+\int_{s}^{t} b\left(\pi_{u} X\right) d B(u) \\
(-\infty<s<t<\infty),
\end{array}
$$

we get

$$
E\left[|X(t)-X(s)|^{2}\right] \leq \gamma_{2}|t-s| \quad(|t-s|<1)
$$

with some constant $\gamma_{2}$ and so

(11. 8) $E\left[\left|a\left(\pi_{t} X\right)-a\left(\pi_{s} X\right)\right|^{2}\right] \leq \gamma_{3}|t-s|$;

$$
E\left[\left|b\left(\pi_{c} X\right)-b\left(\pi_{c} X\right)\right|^{2}\right] \leq \gamma_{3}|t-s| \quad(|t-s|<1),
$$

by virtue of (11.2).

Since $E\left[X(t)^{2}\right]=E\left[\left(X(0)^{2}\right]=\gamma_{0}<\infty, Y(t) \equiv E[X(t) \mid \mathscr{B}(d B)]\right.$ is well defined and so

$$
\begin{aligned}
& E\left[Y(t)^{2}\right] \leq E\left[X(t)^{2}\right]=\gamma_{0} \\
& E\left[|Y(t)-Y(s)|^{2}\right] \leq \gamma_{2}|t-s|
\end{aligned}
$$

by (C. 1$)$ and (11.7), so that $Y(t)$ has a measurable version which we shall denote with $Y(t)$ again. Because of

$$
\begin{aligned}
& E\left\{\left[\int_{-\infty}^{0}\left|\pi_{t} Y(s)\right|\left|d K_{i}(s)\right|\right]^{2}\right\} \\
& \quad \leq\left\|K_{i}\right\| \int_{-\infty}^{0} E\left[Y(t+s)^{2}\right]\left|d K_{i}(s)\right| \leq\left\|K_{i}\right\|^{2} \gamma_{0}<\infty,
\end{aligned}
$$


$\pi_{t} Y(s)$ is integrable with respect to $\left|d K_{i}(s)\right|$ for every $t$ and $i=1,2$. Therefore $a\left(\pi_{t} Y\right)$ and $b\left(\pi_{t} Y\right)$ are well defined and we have (11. 10) $\quad a\left(\pi_{t} Y\right)=E\left[a\left(\pi_{t} X\right) \mid \mathscr{B}(d B)\right], \quad b\left(\pi_{t} Y\right)=E\left[b\left(\pi_{t} X\right) \mid \mathscr{B}(d B)\right]$ by (11.2).

Using (C. 1) and (11.10), we can derive

$$
\begin{aligned}
& E\left[a\left(\pi_{t} Y\right)^{2}\right] \leq \gamma_{1}, \quad E\left[b\left(\pi_{t} Y\right)^{2}\right] \leq \gamma_{1} \\
& E\left[\left|a\left(\pi_{t} Y\right)-a\left(\pi_{s} Y\right)\right|^{2}\right] \leq \gamma_{3}|t-s| \\
& E\left[\left|b\left(\pi_{t} Y\right)-b\left(\pi_{s} Y\right)\right|^{2}\right] \leq \gamma_{3}|t-s|
\end{aligned}
$$

from (11.5) and (11.8). This completes the proof of Theorem 14.

Setting $X=X(t), \mathcal{C}=\mathscr{B}_{-\infty t}(X), \mathscr{D}=\mathscr{B}_{-\infty t}(d B)$ and $\mathscr{E}=\mathscr{B}_{t \infty}(d B)$ in (C. 3), we have

$$
Y(t)=E\left[X(t) \mid \mathscr{B}_{-\infty t}(d B)\right],
$$

so that $Y(t)$ is measurable with respect to $\mathscr{B}_{-\infty t}(d B)$ and $\mathscr{B}_{-\infty t}(Y, d B)$ is therefore independent of $\mathscr{B}_{t \infty}(d B)$. Thus we can see that

$$
I(Y) \equiv \int_{s}^{t} a\left(\pi_{u} Y\right) d u+\int_{s}^{t} b\left(\pi_{u} Y\right) d B(u)
$$

is well-defined.

Let $I(X)$ denote the right side of (11.6), $I_{\Lambda}(X)$ be the approximate sum for $I(X)$ with respect to the division $\Delta \equiv\left(s=u_{0}<u_{1}<\right.$ $\left.\cdots<u_{u}=t\right)$ and $I_{\Lambda}(Y)$ that for $I(Y)$. Then (11.8) and (11.8') imply that, as $\|\Delta\| \equiv \max \left(u_{i}-u_{-1}\right) \rightarrow 0$,

$$
\begin{aligned}
& E\left[\left|I_{\Lambda}(X)-I(X)\right|^{2}\right] \rightarrow 0, \\
& E\left[\left|I_{\Lambda}(Y)-I(Y)\right|^{2}\right] \rightarrow 0 .
\end{aligned}
$$

Observing the forms of $I_{\Lambda}(X)$ and $I_{\Lambda}(Y)$, we get

$$
I_{\Lambda}(Y)=E\left[I_{\Lambda}(X) \mid \mathscr{B}(d B)\right]
$$

and so

$$
\begin{gathered}
E\left\{\left[I_{\Lambda}(Y)-E(I(X) \mid \mathscr{B}(d B))\right]^{2}\right\} \\
\quad \leq E\left[\left(I_{\Lambda}(X)-I(X)\right)^{2}\right] \rightarrow 0
\end{gathered}
$$

by (C. 1) and (11.13), as $\|\Delta\| \rightarrow 0$. Hence it follows by (11.13') and (11.6) that 
On stationary solutions of a stochastic differential equation 51

$$
I(Y)=E[I(X) \mid \mathcal{B}(d B)]=E[X(t)-X(s) \mid \mathcal{B}(d B)]=Y(t)-Y(s) .
$$

Since $X$ and $d B$ are strictly stationarily correlated, the shift operator $\theta_{\tau}$ on $\mathscr{B}(X, d B)$ is measure preserving and $\theta_{\tau}(t)=X(t+\tau)$ and $\theta_{\tau} \mathscr{S}(d B)=\mathscr{B}(d B)$. Hence it follows by (C.2) that

$$
Y(t+\tau)=E(X(t+\tau) \mid \mathscr{B}(d B))=E\left(\theta_{\tau} X(t) \mid \theta_{\tau} \mathscr{B}(d B)\right)=\theta_{\tau} Y(t),
$$

so that $Y(t)$ has a baceward representation by multiple Wiener integrals by virtue of $\mathscr{B}_{-\infty t}(Y) \subset \mathscr{B}_{-\infty t}(d B)$ and $E\left(Y(0)^{2}\right)<\infty$.

Now we shall combine Theorem 14 with Theorem 8 and Theorem 13 to prove

THEOREM 15. Suppose that $a(f)$ and $b(f)$ are of the form (11.2) and set

$$
\begin{gathered}
-c=\text { the jump of } d K_{1} \text { at } 0 \\
c_{1}=\int_{-\infty}^{0-}\left|d K_{1}(t)\right|, \quad c_{2}=\int_{-\infty}^{0}\left|d K_{2}(t)\right| .
\end{gathered}
$$

Then there exists a stationary solution of (11.1) with a backward representation by multiple Wiener integrals integrals in each of the cases:

(i) $c>c_{1}+\frac{1}{2} c_{2}^{2}$ and the supports of $d K_{1}$ and $d K_{2}$ are bounded,

(ii) $c>c_{1}+\frac{1}{4} c_{2}^{2}+\frac{1}{4}\left(c_{2}^{4}+8 c_{1} c_{2}^{2}\right)^{1 / 2}$.

In the case (ii) this solution is the only one solution of (11.1) with $\sup _{t} E\left(X(t)^{2}\right)<\infty$.

Proof: The assertion in the case (i) follows at once from Theorem 8 and Theorem 14 ; notice that $a(f)$ and $b(f)$ are continuous with respect to the $\rho_{-}$-topology, since $d K_{1}$ and $d K_{2}$ have bounded supports. The assertion in the case (ii) and the second part of our theorem follow from Theorem 13 and Theorem 14 (see also Remarks 3 and 4 at the end of Section 10).

\section{Linear Coefficients (2).}

In this section we shall discuss the case that $a(f)$ is linear and $b(f) \equiv 1$. Then our equation turns out to be 


$$
d X(t)=\left[\mu+\int_{-\infty}^{0} X(t+s) d K(s)\right] d t+d B(t)
$$

Before stating our results we shall examine this problem heuristically.

First of all it should be noted that once we get a solution with a backward moving average representation, then it should be canonical, i.e., $\mathscr{B}_{t}(X)=\mathscr{B}_{t}(d B)$ by virtue of Theorem 10 .

Let $X(t)$ be any stationary solution of $(12.1)$ with $E\left(X(0)^{2}\right)<\infty$. If we assume

$$
k \equiv \int_{-\infty}^{0} d K(s) \neq 0,
$$

then the stationary process

$$
Y(t) \equiv X(t)+\frac{\mu}{k}
$$

satisfies

$$
d Y(t)=\left(\int_{-\infty}^{0} Y(t+s) d K(s)\right) d t+d B(t) .
$$

Using the properties of stationary random distributions [7] we can write $(12.1)$ as

$$
\left(12.1^{\prime \prime}\right) \quad D Y(\varphi)=\int_{-\infty}^{\infty} \varphi(t) \int_{-\infty}^{0} Y(t+s) d K(s) d t+D B(\mathscr{P}),
$$

where $D$ is the Schwartz derivative and $\varphi$ moves over the space of all $C^{\infty}$-functions of compact support.

Express the stationary process $Y(t)$ and the stationary random distribution $D B$ in the Fourier transforms :

$$
Y(t)=c+\int_{-\infty}^{\infty} e^{i \lambda t} d M(\lambda)
$$

and

$$
D B(\mathscr{P})=\int_{-\infty}^{\infty} \mathcal{H} \rho(\lambda) d U(\lambda)
$$

where $d M(\lambda)$ is an orthogonal random measure with

$$
\int_{-\infty}^{\infty} E\left[|d M(\lambda)|^{2}\right]<\infty,
$$

$d U(\lambda)$ is a complex Gaussian orthogonal random measure with 
On stationary solutions of a stochastic differential equation 53

$$
\begin{gathered}
E(\mid d U) \lambda)\left.\right|^{2}=\frac{1}{2 \pi} d \lambda \\
U(\Lambda)=\overline{U(-\Lambda)}
\end{gathered}
$$

and $\mathcal{F} \varphi(\lambda)$ is the Fourier transform of $\varphi$ :

$$
\mathcal{F} \mathcal{P}(\lambda)=\int_{-\infty}^{\infty} e^{i \lambda t} \mathcal{P}(\lambda) d \lambda
$$

Then it is easy to see that

and

$$
D Y(\rho)=\int_{-\infty}^{\infty} \mathcal{F} \varphi(\lambda) i \lambda d M(\lambda)
$$

$(12.9)$

$$
\begin{aligned}
\int_{-\infty}^{\infty} & \rho(t) \int_{-\infty}^{0} Y(t+s) d K(s) d t \\
& =\int_{-\infty}^{\infty} \mathcal{F}(\lambda) \mathcal{F} K(\lambda) d M(\lambda)+c \cdot k \int_{-\infty}^{\infty} \mathcal{P}(t) d t,
\end{aligned}
$$

where $\mathscr{F} K(\lambda)$ is the Fourier transform of the signed measure $d K$ :

$$
\mathscr{F} K(\lambda)=\int_{-\infty}^{0} e^{i \lambda t} d K(t)
$$

notice that $\mathscr{F} K(\lambda)$ is bounded:

$$
|\mathcal{F} K(\lambda)| \leq \int_{-\infty}^{0}|d K(t)| \equiv k_{0}<\infty .
$$

Putting (12.8) and (12. $\left.1^{\prime \prime}\right)$, we get

$$
\int \mathcal{F} \mathscr{P}(\lambda) i \lambda d M(\lambda)=\int \mathcal{F}(\lambda) \mathscr{F} K(\lambda) d M(\lambda)+c k \int \mathcal{P}(t) d t+\int \mathcal{F} \mathscr{P}(\lambda) d U(\lambda) \text {, }
$$

i.e.

$$
\begin{aligned}
& c k \int \mathcal{P}(t) d t=0 \quad \text { and so } \quad c=0, \\
& i \lambda d M(\lambda)=\mathcal{F} K(\lambda) d M(\lambda)+d U(\lambda)
\end{aligned}
$$

and so

$$
(i \lambda-\mathscr{F} K(\lambda)) d M(\lambda)=d U(\lambda)
$$

If

$$
i \lambda-\mathcal{F} K(\lambda) \neq 0,
$$

then we get

$$
d M(\lambda)=(i \lambda-\mathscr{F} K(\lambda))^{-1} d U(\lambda)
$$


and it is conceivable that our solution $X(t)$ is expressible in

$$
X(t) \equiv-\frac{M}{k}+\int_{-\infty}^{\infty} e^{i \lambda t}(i \lambda-\mathscr{F} K(\lambda))^{-1} d U(\lambda)
$$

In order for $X(t)$ to be a stationary process, we need the assumption :

$$
\int_{-\infty}^{\infty}|i \lambda-\mathcal{G} k(\lambda)|^{-2} d \lambda<\infty
$$

The following Lemma will give reasonable sufficient conditions for (12.14).

\section{LEMMA 12.1. If}

$$
k=\int_{-\infty}^{0} d K(t)<0
$$

and

$$
k_{1}=\int_{-\infty}^{0}|t||d K(t)|<1,1
$$

then $H(\nu)=i \nu-\int_{-\infty}^{0} e^{i \nu t} d K(t)(\operatorname{Im} \nu \leq 0)$ has the following properties:

(H. 1) $H(\nu)$ is continuous in $\operatorname{Im} \nu \leq 0$ and analytic in $\operatorname{Im} \nu<0$

(H. 2) $H(\nu) \neq 0$ in $\operatorname{Im} \nu \leq 0$ and $\int_{-\infty}^{\infty}|H(\lambda+i \nu)|^{-2} d \lambda$ is bounded in $\mu \leq 0$

(H.3) The inverse Fourier transform of $H(\lambda)$ vanishes on $t \leq 0$.

ProOF : (H. 1) is clear. To prove (H.2), denote the real and imaginary parts of $H(\lambda+i \mu)$ by $R(\lambda, \mu)$ and $I(\lambda, \mu)$ respectively. Then

$$
R(\lambda, \mu)=-\mu-\int_{-\infty}^{0} \cos \lambda t e^{-\mu t} d K(t)
$$

and

$$
I(\lambda, \mu)=\lambda-\int_{-\infty}^{0} \sin \lambda t e^{-\mu t} d K(t) .
$$

It holds for $\mu \leq 0$ that

$$
\frac{\partial R}{\partial \mu}=-1+\int_{-\infty}^{0} t \cos \lambda t e^{-\mu t} d K(t) \leq-1+k_{1}<0
$$

1) These conditions, (K.1) and (K.2), were sought by our conversation with J. McGregor. 
and

$$
\frac{\partial R}{\partial \lambda}=\left|\int_{-\infty}^{0} t \sin \lambda t e^{-\mu t} d K(t)\right| \leq k_{1}
$$

so that

$$
|H(\lambda+i \mu)| \geq R(\lambda, \mu) \geq R(\lambda, 0) \geq R(0,0)-k_{1}|\lambda|=-k-k_{1}(\lambda)
$$

On the other hand

$$
|H(\lambda+i \mu)| \geq|I(\lambda, \mu)| \geq|\lambda|\left(1-k_{1}\right) \quad(\text { by }|\sin \lambda t| \leq|\lambda||t|)
$$

Taking a combination, we get

$$
|H(\lambda+i \mu)| \geq \frac{1-k_{1}}{2}\left(-k-k_{1}|\lambda|\right)+\frac{1+k_{1}}{2}|\lambda|\left(1-k_{1}\right)=\frac{1-k_{1}}{2}(-k+|\lambda|)
$$

This proves (H.2). (H. 3) follows at once from (H.1) and (H.2) by Paley-Wiener's theorem.

Using this Lemma we shall prove

THEOREM 16. Under the conditions (K.1) and (K.2), there exists one and only one stationary solution of $(12.1)$ with $E\left(X(0)^{2}\right)<\infty$.

The solution has a canonical linear backward representation [10]

$$
X(t)=-\frac{\mu}{k}+\int_{-\infty}^{t} g(s-t) d B(s),
$$

where $g(t)$ is a real valued square summable function vanishing on $t \geq 0$ and is given by

$$
g(t)=\frac{1}{2 \pi} \lim _{A \rightarrow \infty} \int_{-\Lambda}^{\Lambda} e^{-i \lambda t} H(\lambda)^{-1} d \lambda \quad(H(\lambda)=i \lambda-\mathscr{F} K(\lambda))
$$

PROOF: The proof of the first part of the theorem is included in the above explanation. The only point we should check is the equivalence of $\left(12.1^{\prime \prime}\right)$ with $\left(12.1^{\prime}\right)$, but we can prove it by noticing that $\left(12.1^{\prime}\right)$ means

$$
Y(t)-Y(s)=\int_{s}^{t} d u \int_{-\infty}^{0} Y(u+v) d K(v)+B(t)-B(s)
$$

and $\left(12.1^{\prime \prime}\right)$ means

$$
\int_{-\infty}^{\infty} Y(u) \mathcal{P}^{\prime}(u) d u=\int_{-\infty}^{\infty} \boldsymbol{P}(u) \int_{-\infty}^{0} Y(u+v) d K(v) d u+\int_{-\infty}^{\infty} B(u) \boldsymbol{P}^{\prime}(u) d u
$$


which can be expressed as the limit of the Riemann sum over $(k / n, k=\cdots-2,-1,0,1,2, \cdots)$ as $n \rightarrow \infty$.

We can also prove the second part by the usual argument except the fact that $g(t)$ is real and vanishes on $t \geq 0$. $g(t)$ is real because

$$
H(-\lambda)=-i \lambda-\int e^{-i \tau t} d K(t)=\overline{H(\lambda)}
$$

and $g(t)$ vanishes on $t \geq 0$, as we can see from (H.3) in Lemma 2.1.

We should mention one word on the continuity of almost all paths of the solution $X(t)$ obtained above. For any given pair $s<t$, we have

(12.17) $X(t)-X(s)=\int_{s}^{t}\left(M+\int_{-\infty}^{0} X(u+v) d K(v)\right) d U+B(t)-B(s)$

with probability 1 , but the usual argument will show that $X(t)$ has a version with continuous paths for which, with probability 1 , (12.17) holds simultaneously for every pair $s<t$.

\section{Diffusions}

In this section we consider the special case that $a(f)$ and $b(f)$ depend only on the value $f(0)$, i.e.

$$
a(f)=\alpha(f(0)), \quad b(f)=\beta(f(0))
$$

Therefore our stochastic differential equation will be of the form:

$$
d X(t)=\alpha(X(t)) d t+\beta(X(t)) d B(t) .
$$

We shall assume the Lipschitz condition

$$
|\alpha(\xi)-\alpha(\eta)|+|\beta(\xi)-\beta(\eta)| \leq K|\xi-\eta| .
$$

The one-sided solution of this equation was treated by K. Itô [8] in connection with the theory of diffusions. Now we shall discuss its stationary solutions.

Before entering our problem, let us examine the nature of the one-sided solutions, using Feller's theory of general one-dimensional diffusions.

Let $P_{\xi}$ be the probability law governing the solution $X(t)$, 
On stationary solutions of a stochastic differential equation 57

$t \geq 0$ with the initial condition $X(0) \equiv \xi$, where $\xi$ is a real constant. Then the system $\left(P_{\xi}, \xi \in R^{1}\right)$ defines a diffusion (=strict Markov process with continuous paths) on $R^{1}$, the domain $\mathscr{D}(\mathcal{G})$ of its generator $\mathcal{G}$ contains the space $\mathcal{C}_{0}^{2}$ of all twice continuously differentiable functions of compact support and

$$
\mathcal{G} u(\xi)=\frac{1}{2} \beta(\xi)^{2} \frac{d^{2} u}{d \xi^{2}}+\alpha(\xi) \frac{d u}{d \xi} \quad u \in \mathcal{C} .
$$

(see K. Itô [8], K. Itô and H. P. McKean, Jr. [9] and E. B. Dynkin [2]).

Our diffusion does not exhibit all different behaviors of Feller's general diffusion; for example, no shunt of the reflecting boundary type can occur, as we can see in

THEOREM 17. Denote the path of our diffusion $\left(P_{\xi}, \xi \in R^{1}\right)$ with $x_{t}$. Then we have the following cases.

( i ) If $\beta(\xi) \neq 0$, then $\xi$ is a non-singular point, i.e.

$$
P_{\xi}\left(x_{t}>\xi \text { and } x_{s}<\xi \text { for some } t, s>0\right)=1 \text {. }
$$

(ii) If $\beta(\xi)=0$ and $(\alpha(\xi)>0$, then $\xi$ is a strict right shunt, i.e.

$$
P_{\xi}\left(x_{t}>\xi \text { for every } t>0\right)=1 \text {. }
$$

(iii) If $\beta(\xi)=0$ and $\alpha(\xi)<0$, then $\xi$ is a strict left shunt, i.e.

$$
P_{\xi}\left(x_{t}<\xi \text { for every } t>0\right)=1 \text {. }
$$

(iv) If $\beta(\xi)=\alpha(\xi)=0$, then $\xi$ is a trap, i.e.

$$
P_{\xi}\left(x_{t}=\xi \text { for every } t \geq 0\right)=1 \text {. }
$$

Proof: As we mentioned above, $P_{\xi}$ is the probability law of of the sample path of the unique solution of the stochastic integral equation:

$$
X(t)=\xi+\int_{0}^{t} \alpha(X(s)) d s+\int_{0}^{t} \beta(X(s)) d B(s) .
$$

In this proof $c_{1}, c_{2}, \cdots$ stand for positive constants and $t$ and $s$ move only on $[0,1]$, unless stated otherwise. For example, $F(t) \leq c_{1} G(t)$ means that there exists a positive constant $c_{1}$ such that $F(t) \leq c_{1} G(t)$ for every $t \in[0,1]$. 
Case $(i) . \quad \beta(\xi) \neq 0$.

Consider a Gaussian process $Y(t)$ :

(13. 6) $Y(t) \equiv \xi+\int_{0}^{t} \alpha(\xi) d s+\int_{0}^{t} \beta(\xi) d B(s)=\xi+\alpha(\xi) t+\beta(\xi) B(t)$.

Then it is clear that

$$
\begin{aligned}
& P\left(Y(t)>\xi+\alpha(\xi) t+|\beta(\xi)| t^{1 / 2}\right) \\
& \quad=P\left(Y(t)<\xi+\alpha(\xi) t-|\beta(\xi)| t^{1 / 2}\right) \\
& \quad=\frac{1}{\sqrt{2 \pi}} \int_{1}^{\infty} \exp \left(-y^{2} / 2\right) d y \equiv c_{1}>0 .
\end{aligned}
$$

If follows from (13.5) that

$$
E\left[(X(t)-\xi)^{2}\right] \leq c_{2} t
$$

as we saw in Section 10 , and so

$$
E\left[(X(t)-Y(t))^{2}\right] \leq c_{3} t^{2}
$$

by virtue of the Lipschitz condition. Hence it follows that

$$
P\left(|X(t)-Y(t)|>t^{3 / 4}\right) \leq c_{3} t^{1 / 2} .
$$

Using (13.7) and (13.10), we get

$$
P\left(X(t)>\xi+\alpha(\xi) t+|\beta(\xi)| t^{1 / 2}-t^{3 / 4}\right)>c_{1}-c_{3} t^{1 / 2}
$$

and

$$
P\left(X(t)<\xi+\alpha(\xi) t-|\beta(\xi)| t^{1 / 2}+t^{3 / 4}\right)>c_{1}-c_{3} t^{1 / 2} .
$$

By taking $t>0$ so small that

$$
t<1, \quad|\beta(\xi)| t^{1 / 2}>|\alpha(\xi)| t+t^{3 / 4} \text { and } c_{1}-c_{3} t^{1 / 2}>0,
$$

we get $P(X(t)>\xi)>0$ and $P(X(t)<\xi)>0$ for some $t$, i.e., $P_{\xi}\left(x_{t}>\xi\right)$ $>0$ and $P_{\xi}\left(x_{t}<\xi\right)>0$ for some $t$. Therefore $\xi$ is a non-singular point and

$$
P_{\xi}\left(x_{t}>\xi \text { and } x_{s}<\xi \text { for some } t, s>0\right)=1 \text {, }
$$

as is well known in the theory of diffusions (see E. B. Dynkin [2], and K. Itô and H. P. McKean, Jr. [9]).

Case $(i i) . \quad \beta(\xi)=0, \alpha(\xi)>0$.

Consider the same $Y(t)$ as in (13.6). Since $\beta(\xi)=0, Y(t)$ is a 
On stationary solutions of a stochastic differential equation 59

fixed linear function :

$$
Y(t)=\xi+\alpha(\xi) t
$$

(13.8) can be sharpened in this case by virtue of $\beta(\xi)=0$. As we saw in Section 10, we have

as before and

$$
E\left[(X(t)-\xi)^{2}\right] \leq c_{2} t
$$

$$
\begin{gathered}
E\left[\alpha(X(t))^{2}\right] \leq c_{3} \\
E\left[\beta(X(t))^{2}\right] \equiv E\left[(\beta(X(t))-\beta(\xi))^{2}\right] \leq K^{2} E\left[(X(t)-\xi)^{2}\right] \leq c_{4} t .
\end{gathered}
$$

Hence it follows that

$$
\begin{aligned}
& E\left[(X(t)-\xi)^{2}\right] \\
& \quad \leq 2 t \int_{0}^{t} E\left[\alpha(X(s))^{2}\right] d s+2 \int_{0}^{t} E\left[\beta(X(s))^{2}\right] d s \\
& \quad \leq 2 c_{3} t^{2}+c_{4} t^{2}=c_{5} t^{2} .
\end{aligned}
$$

Writing $X(t)$ in the form:

$$
\begin{aligned}
X(t) & =Y(t)+(X(t)-Y(t)) \\
& =\xi+\alpha(\xi) t+\int_{0}^{t}(\alpha(X(s))-\alpha(\xi)) d s+\int_{0}^{t}(\beta(X(s))-\beta(\xi)) d B(s) \\
& =\xi+\alpha(\xi) t+I_{1}(t)+I_{2}(t),
\end{aligned}
$$

we shall examine the behavior of $X(t)$ near $t=0$.

Since $\alpha(X(s))-\alpha(\xi)(\varepsilon \downarrow 0)$ with probability 1 , we have

$$
\left|I_{1}(t)\right|<\frac{1}{3} \alpha(\xi) t \quad 0 \leq t \leq \tau_{1}
$$

where $\tau_{1}$ is a certain positive random variable.

Now we shall estimate $I_{2}(t)$ by means of $\left(13.8^{\prime}\right)$. Writing $\bar{I}_{2}(t)$ for $\sup _{0 \leq s \leq t}\left|I_{2}(s)\right|$, we get

$$
\begin{aligned}
P\left(\bar{I}_{2}(t)>\varepsilon\right) & \leq \varepsilon^{-2} \int_{0}^{t} E\left[(\beta(X(s))-\beta(\xi))^{2}\right] d s \\
& \leq \varepsilon^{-2} K^{2} \int_{0}^{t} E\left[(X(s)-\xi)^{2}\right] d s \\
& \leq \varepsilon^{-2} K^{2} c_{5} \frac{t^{3}}{3} c_{6} t^{3} \varepsilon^{-2}
\end{aligned}
$$

and so 


$$
P\left(\bar{I}_{2}(t)>t^{4 / 3}\right) \leq c_{6} t^{1 / 2}
$$

Hence it follows that

$$
\sum_{n} P\left(\bar{I}_{2}\left(2^{-3 n}\right)>2^{-4 n}\right) \leq \sum c_{6} 2^{-n}<\infty,
$$

so that we can apply the Borel-Cantelli lemma to get a positive random variable $\nu$ (which is finite with probability 1 ) such that

$$
\bar{I}_{2}\left(2^{-3 n}\right) \leq 2^{-4 n} \quad \text { for } \quad n \leq \nu .
$$

Then we have

$$
\bar{I}_{2}(t) \leq 2^{4} t^{4 / 3} \quad \text { for } \quad 0 \leq t \leq 2^{-3 \nu}
$$

in fact, taking $m=m(t) \leq \nu$ with

$$
2^{-3(m+1)} \leq t \leq 2^{-3 m},
$$

we can get

$$
I_{2}(t) \leq \bar{I}_{2}\left(2^{-3 m}\right) \leq 2^{-4 m} \leq 2^{4} 2^{-4(m+1)} \leq 2^{4} t^{4 / 3} .
$$

Setting $\tau_{2}=\min \left(3^{-3} 2^{-12} \alpha(\xi)^{3}, 2^{-3 \nu}\right)$, we have

$$
\left|I_{2}(t)\right| \leq \bar{I}_{2}(f) \leq 2^{4} t^{1 / 3} t \leq 2^{4} \tau_{2}^{1 / 3} t \leq \frac{1}{3} \alpha(\xi) t
$$

for $0 \leq t \leq \tau_{2}$.

Putting (13.12) and (13.14) in (13.11) we get

$$
P\left(X(t)>\xi+\frac{1}{3} \alpha(\xi) t \quad \text { for } \quad 0 \leq t \leq \min \left(\tau_{1}, \tau_{2}\right)=1,\right.
$$

a fortiori

$$
P_{\xi}\left(x_{t}>\xi \text { for sufficiently small } t>0\right)=1 .
$$

By the property of the one-dimensional diffusion (see [9] or [2] for example), (13.15) implies the conclusion of (ii).

Case (iii). $\beta(\xi)=0, \alpha(\xi)<0$. The same as (ii).

Case (iv). $\quad \beta(\xi)=\alpha(\xi)=0$. Since $X(t) \equiv \xi$ satisfies $(13.5), P_{\xi}$ is concentrated on the single path identically equal to $\xi$.

Thus Theorem 17 is completely proved.

Now consider the set of all non-singular points. It is a finite or countable sum of disjoint open intervals $I_{n}=\left(i_{n}, j_{n}\right), n=1,2, \cdots$. 
Each $I=I_{n}$ is the maximal non-singular interval on which Feller's scale $d s$ and speed measure $d m$ are given as

$$
d s(\xi)=e^{-\gamma(\xi)} d \xi, \quad d m(\xi)=\beta(\xi)^{-2} e^{\gamma(\xi)} d \xi
$$

with

$$
\gamma(\xi)=\int_{\zeta}^{\xi} \alpha(\eta) \beta(\eta)^{-2} d \eta
$$

where $\zeta$ is any point assigned arbitrarily in $I$.

In the discussion of the stationary solution of our stochastic differential equation (13.2), a non-singular interval $I=(i, j)$ of positive recurrent type is important. It is characterized in terms of the scale $d s$ and the speed measure $d m$ as

$$
\int_{i}^{j} d s=\infty, \quad \int_{\zeta}^{j} d s=\infty, \quad \int_{i}^{j} d m<\infty .
$$

In case both $i$ and $j$ are finite, $(i, j)$ is of such type if $\alpha(i)>0$ and $\alpha(j)<0$, but not so if either $\alpha(i)<0$ or $\alpha(j)>0$.

From now on we shall denote all non-singular intervals of positive recurrent type by $J_{1}, J_{2}, \cdots$ and the scale and the speed measure on each $J_{n}$ by $d s_{n}$ and $d m_{n}$ respectively.

Let $P(t, \xi, E)$ be the transition probability measure of our diffusion $\left(P_{\xi}, \xi \varepsilon(-\infty, \infty)\right)$. A probability measure $\mu$ on the real line called an invariant measure, if

$$
\mu(E)=\int_{-\infty}^{\infty} P(t, \xi, E) d \mu(\xi) .
$$

Hereafter we denote the set of all traps with $\theta$. Take any trap $\theta$. Then the $\delta$-distribution concentrated at $\theta$ is a trivial invariant measure and $X(t) \equiv \theta$ is a trivial stationary solution of our equation (13.2).

Take any non-singular interval $J=J_{n}$ of positive recurrent type. Then the probability measure $d \nu=d_{\nu_{n}}$ proportional to its speed measure $d m=d m_{n}$ is the only one invariant measure concentrated on this interval.

The following lemma 13.1 which will be useful here follows easily from the eigen-differential expansion of $P(t, \xi, E)$ due to H. P. McKean, Jr. [11]. 
LEMMA 13.1. Let $J$ be a non-singular interval of positive recurrent type and $d \nu$ its invariant measure.

(i) Given any $\varepsilon>0$ and any compact subinterval $J^{\prime}$ of $J$, there exists a compact subinterval $J^{\prime \prime}=J^{\prime \prime}\left(\varepsilon, J^{\prime}\right)$ such that

$$
P\left(t, \xi, J^{\prime \prime}\right)>1-\varepsilon
$$

for every $t \geq 0$ and every $\xi \in J^{\prime}$.

(ii) Given any $\varepsilon>0$, any bounded continuous function $h$ defined on $J$ and any compact subinterval $J^{\prime}$, there exists $t_{0}=t_{0}\left(\varepsilon, h, J^{\prime}\right)>0$ such that

$$
\left|\int_{J} h(\eta) P(t, \xi, d \eta)-\int_{J} h(\eta) d \nu(\eta)\right|<\varepsilon
$$

for every $t>t_{0}$ and ever $\xi \in J^{\prime}$.

LEMMA 13.2. Let $J$ be a non-singular interval of positive recurrent type, $J^{\prime}$ a compact subinterval of $J$ and $Y_{\xi}(t)$ the solution of

$$
Y(t)=\xi+\int_{0}^{t} \alpha(Y(s))+\int_{0}^{t} \beta(Y(s)) d B(s) .
$$

Then

$$
E\left(\left|\arctan Y_{\xi_{2}}(t)-\arctan Y_{\xi_{1}}(t)\right|\right) \rightarrow 0
$$

uniformly on $\left(\xi_{1}, \xi_{2}\right) \in J^{\prime} \times J^{\prime}$ as $t \rightarrow \infty$.

ProOF: Since $Y_{\xi}(t)$ can be approximated by successive approximation, $Y_{\xi}(t)$ is $\mathscr{B}_{0, t}(d B)$-measurable. Take $\xi_{1}<\xi_{2}$ in $J$. Let $\sigma$ be $\sup \left\{t: Y_{\xi_{1}}(s)<Y_{\xi_{2}}(s)\right.$ for $\left.s \leq t\right\}$. Using the strong Markov property of the Wiener process $B(t)$ and definition of stochastic integral, we can see that with probability 1 , either $\sigma=\infty$ or

$$
Y(t+\sigma)=Y(\sigma)+\int_{0}^{t} \alpha(Y(s+\sigma)) d s+\int_{0}^{t} \beta(Y(s+\sigma)) d B_{\sigma}(s)
$$

for $Y=Y_{\xi_{1}}, Y_{\xi_{2}}$, where $B_{\sigma}(s)=B(s+\sigma)-B(\sigma)$; we can prove by routine argument that $B_{\sigma}(s), s \geq 0$ is a Wiener process and that $\left[\bigvee_{0 \leq s \leq t} \mathscr{B}\left(Y_{\xi}(s+\sigma)\right)\right] \vee \mathscr{B}_{0 t}\left(d B_{\sigma}\right)$ is independent of $\mathscr{B}_{t \infty}\left(d B_{\sigma}\right)$, so that the stochastic integral in (13.22) is meaningful.

By the uniqueness of the solution of the stochastic integral 
equation and the fact that $Y_{\xi_{2}}(\sigma)=Y_{\xi_{1}}(\sigma)$ if $\sigma<\infty$, we get from (13. 22)

(13.23) $P\left(\right.$ either $\sigma=\infty$ or $Y_{\xi_{2}}(t)=Y_{\xi_{1}}(t)$ for $\left.t \geq \sigma\right)=1$;

see the remark at the end of this section.

Since $Y_{\xi_{2}}(t)>Y_{\xi_{1}}(t)$ for $t<\sigma$, we get

$$
P\left(Y_{\xi_{2}}(t) \geq Y_{\xi_{1}}(t) \text { for every } t \geq 0\right)=1
$$

Since $h(x) \equiv \arctan x$ is continuous, bounded and increasing, it follows from (13.24) that

$$
E\left[\left|h\left(Y_{\xi_{2}}(t)\right)-h\left(Y_{\xi_{1}}(t)\right)\right|\right]=\left|E\left[h\left(Y_{\xi_{2}}(t)\right)\right]-E\left[h\left(Y_{\xi_{1}}(t)\right)\right]\right|
$$

for $\xi_{1} \leq \xi_{2}$. Noticing the symmetry of $\xi_{1}$ and $\xi_{2}$ in this identity, we can see that it is true for $\left(\xi_{1}, \xi_{2}\right) \in J \times J$, a fortiori for $\left(\xi_{1}, \xi_{2}\right) \in J^{\prime}$ $\times J^{\prime}$. To complete the proof, it is enough to observe

$$
\begin{gathered}
\left|E\left[h\left(Y_{\xi_{2}}(t)\right)\right]-E\left[h\left(Y_{\xi_{1}}(t)\right)\right]\right|=\left|\int_{J} h(\xi) P\left(t, \xi_{2}, d \xi\right)-\int_{J} h(\xi) P\left(t, \xi_{1}, d \xi\right)\right| \\
\leq \sum_{n=1}^{2}\left|\int_{J} h(\xi) P\left(t, \xi_{i}, d \xi\right)-\int_{J} h(\xi) d \nu(\xi)\right|
\end{gathered}
$$

and to use Lemma 13.1(ii).

Using the equivalence in law of $(B(t), t \geq 0)$ and $(B(t-T)-$ $B(-T)$ ) and the fact that the one-sided solution of our stochastic differential equation is determined by successive approximation, we can derive the following lemma immediately from Lemma 13.2.

LEMMA 13.3. Let $J$ be a non-singular interval of positive recurrent type, $J^{\prime}$ a compact subinterval of $J$ and $X_{T, \xi}(t)$ the solution of

$$
X(t)=\xi+\int_{-T}^{t} \alpha(X(s)) d s+\int_{-T}^{t} \beta(X(s)) d B(s) .
$$

Then

$$
E\left[\left|\arctan X_{T, \xi_{2}}(t)-\arctan X_{T, \xi_{1}}(t)\right|\right] \rightarrow 0
$$

uniformly in $\left(\xi_{1}, \xi_{2}\right) \in J^{\prime} \times J^{\prime}$ as $t \rightarrow \infty$.

Now we shall discuss the stationary solution on a single nonsingular interval of positive recurrent type.

THEOREM 18. If $J$ be a non-singular interval of positive re- 
current type, then the stochastic differential equation (13.2) has one and only one solution whose sample path is confined in J with probability 1.

This solution satisfies

$$
\mathscr{B}_{-\infty t}(X)=\mathscr{B}_{-\infty t}(d B) \quad-\infty<t<\infty .
$$

Furthermore if the invariant measure on $J$ has finite second order moment, then this solution has a properly canonical backward representation by multiple Wiener integrals.

\section{PROOF :}

(i) Existence. We shall consider the process $X_{T, \xi}(t), t \geq-T$, introduced in Lemma 13.2. Take a point $\zeta \in J$ and fix it. The limit of $X_{T, \zeta}(t)$ as $T \rightarrow \infty$ is supposed to be a solution.

First of all we shall prove that $X_{T, \zeta}(t)$ converges to a certain random variable $X(t)$ in probability for each $t$.

Take $S$ and $T$ with $-S<-T<t$. Then

$$
X_{S, \zeta}(t)=X_{S, \zeta}(-T)+\int_{-T}^{t} \alpha\left(X_{S, \zeta}(s)\right) d s+\int_{-T}^{t} \beta\left(X_{S, \eta}(s)\right) d B(s) .
$$

Since $X_{S, \zeta}(-T)$ is independent of $\mathscr{B}_{-T_{\infty}}(d B)$, we can obtain $X_{S, \zeta}(t)$ by replacing $\xi$ in $X_{T, \xi}$ with $X_{S, \zeta}(-T)$ by virtue of the uniqueness of the one-sided solution and the Borel-measurability of $X_{T, \xi}(t, \omega)$ in $(\xi, \omega)$ on the product measurable space $R^{1}\left(\mathscr{B}\left(R^{1}\right)\right) \times \Omega(\mathscr{B}(d B)$ ) (see K. Itô [8]). Noticing that $X_{T, \zeta}(t)$ is $\mathscr{B}_{-T_{\infty}}(d B)$-measurable, we get

$$
\begin{aligned}
E[ & {\left[\left|\arctan X_{S, \zeta}(t)-\arctan X_{X, \zeta}(t)\right|\right] } \\
& =\int_{J} P\left(X_{S, \zeta}(-T) \in d \xi\right) E\left[\left|\arctan X_{T, \xi}(t)-\arctan X_{T, \zeta}(t)\right|\right] \\
& =\int_{J} P(S-T, \zeta, d \xi) E\left[\left|\arctan X_{T, \xi}(t)-\arctan X_{T, \zeta}(t)\right|\right] .
\end{aligned}
$$

Using Lemma 13.1 (i) and Lemma 13.3, we can easily see that this tends to 0 as $S>T \rightarrow \infty$. Therefore the limit in probability of $\arctan X_{T, \zeta}(t)$ as $T \rightarrow \infty$ exists, so that $X_{T, \zeta}(t)$ tends to a certain random variable $\tilde{X}(t)$ in probability as $T \rightarrow \infty$. It is clear that $\tilde{X}(t)$ is $\mathscr{B}_{-\infty t}(d B)$-measurable, so that (13.27) holds for $X=\tilde{X}$.

To prove that $\tilde{X}(t)$ has a version with continuous paths which 
On stationary solutions of a stochastic differential equation 65

is a stationary solution of (13.2), we shall express $\widetilde{X}(t)$ by the limit of another sequence of one-sided solution.

Take a random variable $A$ which is governed by the invariant distribution $\nu$ on $J$, by extending the basic probability measure space if necessary. Let $X_{T, A}(t), t \geq-T$, denote the solution of

$$
X(t)=A+\int_{-T}^{t} \alpha(X(s)) d s+\int_{-T}^{t} \beta(X(s)) d B(s) .
$$

It is easy to see that if $S>T$, then

$$
\left(X_{S, A}(t), t \geq-T, \quad B(v)-B(u), \quad v>u \geq-T\right)
$$

is equivalent in law with

$$
\left(X_{T, A}(t), t \geq-T, \quad B(v)-B(u), \quad v>u \geq-T\right)
$$

because $A$ is $\nu$-distributed. This is a nice property of $X_{T, A}$ which $X_{T, \zeta}(t)$ did not enjoy; on the other hand $X_{T, \zeta}(t)$ was $\mathscr{B}_{-T, t}(d B)_{-}$ measurable, while $X_{T, A}$ is not so.

Now we can prove that for each $t, X_{T, A}(t)$ also converges to $\widetilde{X}(t)$ in probability as $T \rightarrow \infty$, by observing, as above,

$$
\begin{aligned}
& E\left[\left|\arctan X_{T, A}(t)-\arctan X_{T, \zeta}(t)\right|\right] \\
& \quad=\int_{J} P(A \in d \xi) E\left[\left|\arctan X_{T, \xi}(t)-\arctan X_{T, \zeta}(t)\right|\right] \\
& \quad=\int_{J} \nu(d \xi) E\left[\left|\arctan X_{T, \xi}(t)-\arctan X_{T, \zeta}(t)\right|\right] \\
& \quad \rightarrow 0 \quad(T \rightarrow \infty),
\end{aligned}
$$

by virtue of Lemma 13. 3 .

Take any time points $t_{1}<t_{2}<\cdots<t_{n}$. Then the joint distribution of

$$
\left(\widetilde{X}\left(t_{1}\right), \cdots, \tilde{X}\left(t_{n}\right), B\left(t_{2}\right)-B\left(t_{1}\right), \cdots, B\left(t_{n}\right)-B\left(t_{n-1}\right)\right)
$$

is clearly the weak* limit of that of

$$
\left(X_{T, A}\left(t_{1}\right), \cdots, X_{T, A}\left(t_{n}\right), B\left(t_{2}\right)-B\left(t_{1}\right), \cdots, B\left(t_{n}\right)-B\left(t_{n-1}\right)\right)
$$

as $T \rightarrow \infty$. But the latter one is independent of $T$ as far as $-T<t_{1}$ because of the nice property of $X_{T, A}$ mentioned above. 
This means that any finite dimensional joint distribution of the system

$$
(\widetilde{X}(t), B(v)-B(u),-\infty<t<\infty,-\infty<u<v<\infty)
$$

is the same as the corresponding joint distribution of

$$
\left(X_{T, A}(t), B(v)-B(u),-\infty<t<\infty,-\infty<u<v<\infty\right)
$$

for big enough $T$ which depends on the time points referred to in the joint distribution.

Since $X_{T, A}(t), t \geq-T$ is continuous in $t$ with probability 1 , it is strictly stationarily correlated to $d B(t), t \geq-T$, and it satisfies (13.2) on $t \geq-T$, we can use routine argument to prove that $\tilde{X}(t)$ has a version $X(t)$ with continuous paths and that it is a stationary solution of (13.2). It is clear that $X(t)$ has also the property $\mathscr{B}_{-\infty t}(X) \subset \mathscr{B}_{-\infty t}(d B)$ as a version of $\tilde{X}(t)$.

Hence it follows by Theorem 10 in Section 9 that $\mathscr{B}_{-\infty t}(X)$ $=\mathscr{B}_{-\infty t}(d B)$.

If $\nu$ has finite second order moment, then $E\left(X(t)^{2}\right)<\infty$, so that $X(t)$ has a backward representation by multiple Wiener integral which is properly canonical by $\mathscr{B}_{-\infty t}(X)=\mathscr{B}_{-\infty t}(d B)$.

(ii) Uniqueness. Take any stationary solution $Y(t)$. Then it is clear that $Y(t)$ is $\nu$-distributed. Since it satisfies

$$
Y(t)=(-T)+\int_{-T}^{t} \alpha(Y(s)) d s+\int_{-T}^{t} \beta(Y(s)) d B(s)
$$

and $Y(-T)$ is independent of $\mathscr{B}_{-T_{\infty}}(d B)$, we get

$$
\begin{aligned}
E & {\left[\left|\arctan Y(t)-\arctan X_{T, \zeta}(t)\right|\right] } \\
\quad & =\int_{J} \nu(d \xi) E\left[\left|\arctan X_{T, \xi}(t)-\arctan X_{T, \zeta}(t)\right|\right]
\end{aligned}
$$

as above. This proves that $P(Y(t)=X(t))=1$ for each $t$ and so $P(Y(t)=X=(t)$ for every $t)=1$ by virtue of the continuity of the paths.

Take any invariant probability measure $\mu$. It is easy to see that $\mu$ can be uniquely decomposed as a convex comaination of the extremal invariant measures introduced above: 


$$
\mu(\cdot)=\int_{\Theta} \delta_{\theta}(\cdot) d k(\theta)+\sum_{n} k_{n} \nu_{n}(\cdot),
$$

where $d k \geq 0, k_{n} \geq 0$ and $\int_{\Theta} d k(\theta)+\sum k_{n}=1$.

For the convenience of notations, we shall take a point $\zeta_{n}$ from each $J_{n}$ as a representative, and write (13.30) as

$$
\mu(\cdot)=\int_{\Theta \vee \Pi} \nu_{\zeta}(\cdot) d k(\zeta),
$$

where $\Pi=\left\{\zeta_{1}, \zeta_{2}, \cdots\right\}, k$ on $\Theta$ is as in (13.30), $k\left(\left\{\xi_{n}\right\}\right)=k_{n}$, and $\nu_{\zeta}=\delta_{\zeta}$ or $\nu_{n}$ according as $\zeta \in \Theta$ or $\zeta=\zeta_{n}$. The expression (13.30) is called the canonical decomposition of $\mu$ and $d k$ is called the coefficient measure.

Let $\Phi$ be the mapping: $\Theta \vee\left(\bigvee_{n} J_{n}\right) \rightarrow \Theta \vee$ II defined as

$$
\Phi(\zeta)=\zeta \quad(\zeta \in \Theta), \quad=\zeta_{n} \quad\left(\zeta \in J_{n}\right) .
$$

Let $X_{\zeta}(t), \zeta \in \Theta \vee I I$ denote the extremal stationary solution we introduced above, i.e.,

$$
\begin{aligned}
& X_{\zeta}(t) \equiv \zeta \\
& (\zeta \in \Theta) \\
& =\text { the stationary determined for } J_{n} \text { in Theorem } 18 \\
& \left(\zeta=\zeta_{n} \in \Pi\right) \text {. }
\end{aligned}
$$

Now we shall prove the following theorem which gives a complete description of stationary solutions.

THEOREM 19.

(i) Let $X(t)$ be any stationary of (13.2) and set $Z=\Phi(X(0))$. Then

(a) $P(Z=\Phi(X(t))$ for every $t)=1$

(b) $\mathscr{B}(Z)=\bigwedge_{t} \mathscr{S}_{-\infty t}(X) \quad(=$ the remote past Borel algebra $)$

(c) $Z$ is independent of $\mathcal{B}(d B)$.

(d) $\mu$ has a canonical decomposition with the coefficient measure $k(\cdot)=P(Z \in \cdot)$ :

$$
\mu(E)=P(X(t) \in E)=\int \nu_{\zeta}(E)(P Z \in d \zeta)
$$

(e) $P\left(X(t)=X_{Z}(t)\right.$ for every $\left.t\right)=1$ 
(ii) Consider any invariant probability measure $\mu$ and decompose it as $\left(13.30^{\prime}\right)$. Take a random variable $Z$ which is independent of $\mathscr{B}(d B)$ and $k$-distributed by extending the basic probability measure space if necessary. Then $X(t) \equiv X_{Z}(t)$ is a stationary solution of (13.3) with $P(X(t) \in E)=\mu(E)$.

Furthermore if $\mu$ has finite second order moment, then this solution has a backward representation by multiple Wiener integrals whose integrands contain $Z$ as a parameter. If and only if $\mu$ is extremal, $X$ has a properly canonical backward representation by multiple Wiener integrals.

PROOF of $(i)$.

(a) is clear by the definitions.

(b) follows at once from (a) by virtue of Theorem 18.

(c) follows at once from (b), because $\mathscr{B}_{-\infty t}(X)$ is independent of $\mathscr{B}_{t \infty}(d B)$.

(d) is clear by the definition of $\Phi$.

(e) Consider the solution $X_{T, \xi}(t)$ of

$$
X_{T, \xi}(t)=\xi+\int_{-T}^{t} \alpha\left(X_{T, \xi}(s)\right) d s+\int_{-T}^{t} \beta\left(X_{T, \xi}(s)\right) d B(s)
$$

If $\xi \in \Theta$, then $X_{T, \xi}(t) \equiv \xi \equiv X_{\xi}(t)$. If $\xi \in J_{n}$, then $X_{T, \xi} \rightarrow X_{\xi}(t)$ in probability as we proved in Theorem 18 .

Since $P\left(Z \in \Theta \vee\left(\bigvee_{n} J_{n}\right)\right)=1$ and $Z$ is independent of $\mathscr{B}(d B)$, $X_{T, Z}(t) \rightarrow X_{Z}(t)$ in probability as far as $t \geq-T$.

It is also clear that $X_{T, X(-T)}(t)=X(t)(t \geq-T)$. Observing

$$
\begin{aligned}
E[ & \mid \arctan X\left(t\left(-\arctan X_{T, Z}(t) \mid\right]\right. \\
& =\sum_{n} E\left[\left|\arctan X_{T, X(-T)}-\arctan X_{T, Z}(t)\right|, X(-T) \in J_{n}\right] \\
& =\sum_{n} E\left[\left|\arctan X_{T, X(-T)}-\arctan X_{T, \zeta_{n}}(t)\right|, X(-T) \in J_{n}\right] \\
& =\sum_{n} \int_{J_{n}} P(X(-T) \in d \xi) E\left[\left|\arctan X_{T, \zeta}-\arctan X_{T, \zeta_{n}}\right|\right] \\
& =\sum_{n} \int_{J_{n}} \mu(d \xi) E\left[\left|\arctan X_{T, \xi}-\arctan X_{T, \zeta_{n}}\right|\right] \\
& =\sum_{n} k_{n} \int_{J_{n}} \nu_{n}(d \xi) E\left[\left|\arctan X_{T, \xi}-\arctan X_{T, \zeta_{n}}\right|\right] \\
& \rightarrow 0,
\end{aligned}
$$

we can see that $X(t)=X_{Z}(t)$. 
On stationary solutions of a stochastic differential equation 69

PROOF of (ii).

Using the process $X_{T, \xi}(t)$ as above, we have

$$
X_{Z}(t)=\lim \operatorname{in}_{T \rightarrow \infty} \text { probability } X_{T, Z} .
$$

Consider a random variable $A$ which is $\mu$-distributed and independent of $\mathscr{B}(d B)$. By the argument used above, we get

$$
E\left[\left|\arctan X_{T, A}(t)-\arctan X_{T, Z}(t)\right|\right] \rightarrow 0
$$

as $t \rightarrow \infty$, and so

$$
X_{Z}(t)=\lim \text { in probability } X_{T, Z}(t) .
$$

The rest of the proof is the same as in the proof of the existence part of Theorem 18; notice that $X_{Z}(t)$ is already continuous in $t$ with probability 1 by the definition.

The statement for the backward representation is now easy to see.

Remark. Let us give a detailed proof of (13.23). Since $Y_{\xi}(t)$ is a function of $\xi, t, B$, we shall write it as $f(\xi, t, B)$. Because of the uniqueness of the solution of (13.20), it is clear that, with probability 1 ,

$$
f(\xi, s+t, B)=f\left(f(\xi, s, B), t, B_{s}\right)
$$

where $B_{s}(t)=B(t+s)-B(s)$.

Since $f(\xi, t, B)$ is $B_{0 t}(B)$-measurable, the event $(\sigma<t)$, is also $\mathscr{S}_{0 t}(B)$-measurable. Approximating $\sigma$ with discrete-valued random variables $\sigma_{n}=[n \cdot \sigma] / n$ as we usually do in deriving the strict Markov property from the Markov property in the Feller process (see [9] or [2]), we can easily see that for any fixed $\xi$, with probability 1 ,

$$
f(\xi, \sigma+t, B)=f\left(f(\xi, \sigma, B), t, B_{\sigma}\right) \quad(t \geq 0)
$$

by interpreting both sides as $\infty$ for convention when $\sigma=\infty$. Therefore with probability 1 ,

$$
\begin{aligned}
f\left(\xi_{1}, \sigma+t, B\right) & =f\left(f\left(\xi_{1}, \sigma, B\right), t, B_{\sigma}\right) \\
& =f\left(f\left(\xi_{2}, \sigma, B\right), t, B_{\sigma}\right) \\
& =f\left(\xi_{2}, \sigma+t, B\right),
\end{aligned}
$$

which proves (13.23). 


\section{A Modified Girsanov Example}

In the general cases treated in Sections 6,7 and 8 we neither proved nor disproved

$$
\mathscr{B}_{-\infty t}(X) \subset \mathscr{B}_{-\infty s}(X) \wedge \mathscr{B}_{s t}(d B) \quad(s<t)
$$

for the stationary solutions obtained there, while (14.1) was true for the special cases discussed in Sections 10, 11, 12 and 13.

Let us consider the equation :

$$
\begin{aligned}
& d X(t)=h(X(t)) d B(t) \\
& \left(h(\xi)=\frac{|\xi|^{\infty}}{1+|\xi|^{\infty}}+|\xi|, \quad \alpha=\text { positive constant }<\frac{1}{2}\right)
\end{aligned}
$$

and call it a modified Girsanov equation. We put an extra term $|\xi|$ in $h(\xi)$ in the Girsanov equation [3] in order to be able to get a stationary solution without any essential change of $h(\xi)$ near $\xi=0$.

First of all we shall consider two solutions of the stochastic integral equation :

$$
X(t)=\int_{0}^{t} h(X(s)) d B(s)
$$

One is the trivial one $X^{0}(t) \equiv 0$. The other one is a Girsanov solution $X^{1}(t)$ which corresponds to the diffusion with the scale $d s=d \xi$ and the speed measure $2 h(\xi)^{-2} d \xi$; Girsanov also constructed a solution which corresponds to the diffusion with the speed measure $2 h(\xi)^{-2} d \xi+c \cdot \delta_{0}$ for every $c>0$, but we will not use this here.

Now we shall combine these two solutions by Ikeda's method (see Ikeda's example [9]).

Take two random variables $\tau^{0}, \tau^{1}$ which are exponentially distributed with mean $\lambda^{0}$ and $\lambda^{1}$ respectively.

Take a countable number of copies $\left(X_{n}^{i}, B_{n}^{i}, \tau_{n}^{i}\right), n=1,2, \cdots$ of $\left(X^{i}, B^{i}, \tau^{i}\right)$ for $i=0,1$. We shall also assume that $\mathcal{B}\left(X_{n}^{i}, B_{n}^{i}, \tau_{n}^{i}\right)$, $n=1,2, \cdots, i=0,1$, are independent.

Let $T(t)$ be the local time at 0 for the process $X_{n}^{1}$. It is clear that $X_{n}^{1}\left(T^{-1}\left(t_{n}^{1}\right)\right)=0$. In view of this fact, we shall define $\left(X_{+}, B\right)$ as follows : 


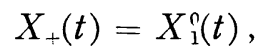

$$
\begin{aligned}
& B(t)=B_{1}^{0}(t), \\
& \left(0 \leq t \leq \tau_{1}^{0}\right) \\
& X_{+}\left(t+\tau_{1}^{0}\right)=X_{1}^{1}(t), \\
& B\left(t+\tau_{1}^{0}\right)-B\left(\tau_{1}^{0}\right)=B_{1}^{1}(t), \\
& \left(0 \leq t \leq T^{-1}\left(\tau_{1}^{1}\right) \equiv \widetilde{\tau}_{1}^{1}\right) ; \\
& X_{+}\left(t+\tau_{1}^{0}+\widetilde{\tau}_{1}^{1}\right)=X_{2}^{0}(t), \quad B\left(t+\tau_{1}^{0}+\widetilde{\tau}_{1}^{1}\right)-B\left(\tau_{1}^{0}+\widetilde{\tau}_{1}^{1}\right)=B_{2}^{0}(t), \\
& X_{+}\left(t+\tau_{1}^{0}+\widetilde{\tau}_{1}^{1}+\tau_{2}^{0}\right)=X_{2}^{1}(t), \\
& \left(0 \leq t \leq \tau_{2}^{0}\right) \text {; } \\
& B\left(t+\tau_{1}^{0}+\widetilde{\tau}_{1}^{1}+\tau_{2}^{0}\right)-B\left(\tau_{1}^{0}+\widetilde{\tau}_{1}^{1}+\tau_{2}^{0}\right)=B_{2}^{1}(t), \\
& \left(0 \leq t \leq T^{-1}\left(\tau_{2}^{1}\right) \equiv \widetilde{\tau}_{2}^{1}\right)
\end{aligned}
$$

and so on. Then $B(t)$ is a Wiener process and (14.3) holds for the pair $\left(X_{+}, B\right)$.

Because of the property of the non-singular diffusion, the set of zero points of $X_{n}^{1}(t)$ is non-dense for every $n=1,2, \cdots$. Therefore $\tau_{1}^{0}, \tau_{2}^{0}, \cdots$ are determined as the lengths of the successive zero intervals of $X_{+}(t)$, so that $\mathscr{B}\left(X_{+}\right) \supset \mathscr{B}\left(\tau_{1}^{0}, \tau_{2}^{n}, \cdots\right)$. On the other hand the construction (14) shows that $\mathscr{B}\left(\tau_{1}^{0}, \tau_{2}^{0}, \ldots\right)$ is independent of $\mathcal{B}(d B)$.

Noticing that the probability measures $P\left(X_{+}(t) \in \cdot\right), t \geq 0$, form a totally bounded set, we can apply the shifting and averaging method used in Secrion 6 to our pair $\left(X_{+}, B\right)$ in order to get a stationary solution $X$ of (14.2).

After the shift the lengths $\tau_{1}^{0}, \tau_{2}^{0}, \cdots$ of zero intervals $\subset[0, \infty)$ of the solution are independently and exponentially distributed with the mean $\lambda^{0}$ and independent of the Wiener process $B$, so that this fact remains to be true even after the averaging. This means that we have, for the stationary solution $X$ obtained iu such a way,

$$
\mathscr{B}_{0 \infty}(X)-\mathscr{B}_{-\infty 0}(X) \supset \mathscr{B}\left(\tau_{1}^{0}, \tau_{2}^{0}, \cdots\right)
$$

and

$$
\mathscr{B}\left(\tau_{1}^{0}, \tau_{2}^{0}, \cdots\right) \text { is independent of } \mathscr{B}_{0 \circ}(d B) \text {, }
$$

so that

$$
\mathscr{B}_{0 \infty}(X) \mp \mathcal{B}_{-\infty 0}(X) \vee \mathcal{B}_{0 \infty}(d B),
$$

This implies that (14.1) does not hold for any pair $s<t$, because $X$ and $d B$ are stationarily correlated. 


\section{A Deterministic Example}

Since we did not require $b(f) \neq 0$ in our stochastic differential equation :

$$
d X(t)=a\left(\pi_{t} X\right) d t+b\left(\pi_{t} X\right) d B(t),
$$

even the deterministic equation (differential-difference equation):

$$
d X(t)=a\left(\pi_{t} X\right) d t
$$

i.e.

$$
\frac{d X(t)}{d t}=a\left(\pi_{t} X\right)
$$

lies in our frame.

Take an example

$$
\frac{d X(t)}{d t}=-X\left(t-\frac{\pi}{2}\right)
$$

The one sided solution is uniquely determined; in fact, if $X(t)$ is given for $t \leq 0$, then $X(t)$ is determined for $0 \leq t \leq \pi / 2$ by integration and then for $\pi / 2 \leq t \leq \pi$ and so on. Besides the trivial stationary solution $X(t) \equiv 0$, we have another stationary solution

$$
X(t)=\sin (\omega+t+\alpha) \quad(\alpha=\text { constant })
$$

where $\omega$ is a probability parameter moving on $\Omega=[0, \pi / 2]$ associated with the uniform distribution. This stationary process is ergodic.

\section{A Two-dimensional Example}

Consider a two-dimensional stochastic differential equation :

$$
\begin{gathered}
d X=\frac{X}{2} d t-Y d B_{2} \\
d Y=\frac{Y}{2} d t+X d B_{2} \quad\left(R \equiv \sqrt{X^{2}+Y^{2}} \leq 1\right) \\
d X=\left(\frac{X}{\sqrt{R}}-\frac{X}{2}\right) d t+\left(X-\frac{X}{R}\right) d B_{1}-Y d B_{2} \\
d Y=\left(\frac{Y}{\sqrt{R}}-\frac{Y}{2}\right) d t+\left(Y-\frac{Y}{R}\right) d B_{1}+X d B_{2}
\end{gathered}
$$


On stationary solutions of a stochastic differential equation 73

We can easily see that the coefficients satisfy the Lipschitz condition in the whole plane, so that the one-sided solution can be determined uniquely by successive approximation.

In order to fined out a stationary solution, we shall write the equations in the polar coordinates $(R, \Theta) .(16.1)$ and (16.2) are transformed into

$$
\begin{gathered}
d R=R \cdot d t \quad(R \leq 1), \quad d R=\sqrt{R} d t+(R-1) d B_{1} \quad(R \geq 1) \\
d \Theta=d B_{2}(\bmod 2 \pi)
\end{gathered}
$$

Observing the form of these equations, we can easily see that

(16.5) $\Theta(t)$ corresponds to a Brownian motion on the unit circle,

(16.6) $R(t)$ corresponds to a one-dimensional diffusion,

and

(16.7) $R(t)$ and $\Theta(t)$ are independent processes.

Using Theorem 17 in Section 13, we can easily see that for the diffusion $R(t), 0$ is a trap, every point in $(0,1]$ is a strict right shunt and $(1, \infty)$ is a non-singular interval of positive recurrent type.

If $(X(t), Y(t))$ is a stationary solution of (16.1) and (16.2), then the corresponding $R(t)$ and $\Theta(t)$ should be stationary solutions of (16.3) and (16.4) respectively. Therefore either $R(t) \equiv 0$ or $R(t)$ is a unique stationary solution with the invariant measure $d \mu(r)$ proportional to the speed measure of the non-singular interval $(1, \infty)$.

$R(t) \equiv 0$ corresponds to the trivial stationary solution $(X(t)$, $Y(t)) \equiv(0,0)$.

The second stationary solution $R(t)$ corresponds to a stationary solution $(X(t), Y(t)) \equiv(R(t), \Theta(t))$ with the invariant measure $d \mu(r)$. $d \theta / 2 \pi$ concentrated in the outside of the unit circle.

We shall verify for this stationary solution

$$
\begin{gathered}
\bigwedge_{t} \mathscr{B}_{-\infty t}(X, Y)=\mathscr{N} \quad(=\text { trivial algebra }) \\
\mathscr{B}_{-\infty t}(X, Y) \subset \mathscr{B}_{-\infty s}(X, Y) \vee \mathscr{S}_{s t}\left(d B_{1}, d B_{2}\right) \quad(-\infty<s<t<\infty)
\end{gathered}
$$

and

$$
\mathscr{B}_{-\infty t}(X, Y) \nsubseteq \mathscr{B}_{-\infty t}\left(d B_{1}, d B_{2}\right) \quad(-\infty<t<\infty)
$$


The transition probability of $R(t)$ tends to its invariant distribution as $t \rightarrow \infty$, and a similar fact holds for $\Theta(t)$. Since $R(t)$ and $\Theta(t)$ are independent, it is also true for the joint process $(R(t), \Theta(t))=(X(t), Y(t))$. Using this, we can verify (16.8) easily.

(16.9) follows at once from the fact that the one-sided solution can be uniquely determined by successive approximation.

To prove (16.10), it is enough to prove it for $t=0$ by virtue of the stationarity. If $\mathscr{B}_{-\infty_{0}}(X, Y) \subset \mathscr{B}_{-\infty_{0}}\left(d B_{1}, d B_{2}\right)$ holds, then we have

$$
E\left[\left|\frac{X(t)}{R(t)}-E\left(\frac{X(t)}{R(t)} \mid \mathscr{B}_{0 t}\left(d B_{1} d B_{2}\right)\right)\right|\right] \rightarrow 0 \quad\left(R=\sqrt{X^{2}+Y^{2}}\right)
$$

as $t \rightarrow \infty$ by Theorem 8 in Section 9 ; notice here that $R(t) \geq 1$ and that $|X(t) / R(t)| \leq 1$

$$
\begin{aligned}
E\left(\frac{X(t)}{R(t)} \mid \mathcal{B}_{0 t}\left(d B_{1}, d B_{2}\right)\right) & \left.=E\left[\cos \left(\Theta(0)+B_{2}(t)-B_{2}(0)\right) \mid \mathcal{S}_{0 t}\left(d B_{1}, d B_{2}\right)\right)\right] \\
& =\frac{1}{2 \pi} \int_{0}^{2 \pi} \cos \left(\theta+B_{2}(t)-B_{2}(0)\right) d \theta=0 ;
\end{aligned}
$$

recall that $\Theta(0)$ is independent of $\mathscr{B}_{0 \infty}\left(d B_{1}, d B_{2}\right)$. Therefore we get

$$
E\left[\left|\frac{X(t)}{R(t)}\right|\right] \rightarrow 0 \quad(t \rightarrow \infty)
$$

and similarly

$$
E\left[\left|\frac{Y(t)}{R(t)}\right|\right] \rightarrow 0,
$$

and so $E[(|X(t)|+|Y(t)|) / R(t)] \rightarrow 0$ in contradiction with $|X|+|Y|$ $\geq R$. Thus (16.10) is proved.

Department of Mathematics, Stanford University and

Division of Applied Mathematics, Brown University

\section{BIBLIOGRAPHY}

[1] J. L. Doob : Stochastic processes, New York 1953.

[2] E. B. Dynkin : Markov processes, 2nd Vol. Moscow (1962), English translation to be published by Springer, Berlin.

[3] I. V. Girsanov : An example of non-uniqueness of the solution of the stochastic equation of K. Itô. Th. Prob. Appl. 7 (1962), 325-331. 
[4] K. Itô : On a formula concerning stochastic differentials, Nagoya Math. Jour. 3 (1951), 55-65.

[5] K. Itô : Multiple Wiener integral, Jour. Math. Soc. Japan 3 (1951), 157-169.

[6] K. Itô : Complex multiple Wiener integral. Jap. Jour. Math. 22 (1952), 63-86.

[7] K. Itô : Stationary random distributions, Memoirs Fac. Science, Kyoto Univ. Ser. A. Math. 28 (1953), 209-223.

[8] K. Itô : On stochastic differential equations, Mem. Amer. Math. Soc. 4 (1954).

[9] K. Itô and H. P. McKean, Jr. : Diffusion processes and their sample paths, to be published by Springer, Berlin.

[10] K. Karhunen : Über die Struktur stationären zufälliger Funktionen. Arkiv för mat. 1 (1950), 114-160.

[11] H. P. McKean, Jr. : Elementary solutions for certain parabolic partial differential equations, Trans. Amer. Math. Soc. 82 (1956), 519-548.

[12] M. Nisio : Remark on the canonical representation of strictly stationary processes, Jour. Math. Kyoto Univ. 1 (1961), 129-146.

[13] Yu V. Prohorov : Convergence of random processes and limit theorems in probability theory, Th. Prob. Appl. 1 (1956), 157-214 (English translation).

[14] M. Rosenblatt: Stationary processes as shifts of functions of independent random variables, Jour. Math. Mech. 8 (1959), 665-682.

[15] M. Rosenblatt: Stationary Markov chains and independent random variables, Jour. Math. Mech. 9 (1960), 945-950.

[16] M. Rosenblatt: The representation of a class of two state stationary processes in terms of independent random variables, Jour. Math. Mech. 12 (1963), 721-730.

[17] A. V. Skorokhod : Limit theorems for stochastic processes, Th. Prob. Appl. 1 (1956), 261-290 (English translation).

[18] A. V. Skorokhod : On the existence and uniqueness of solutions of stochastic differential equations (Russian), Sibirsk. Mat. Z̃. 2 (1961), 129-137.

[19] N. Wiener : Non-linear problems in random theory, Cambridge, Mass. 1958. 